$$
\begin{aligned}
& \text { Aus der Abteilung Urologie } \\
& \text { (Prof. Dr. med. R.-H. Ringert) } \\
& \text { im Zentrum Chirurgie }
\end{aligned}
$$$$
\text { der Medizinischen Fakultät der Universität Göttingen }
$$

\title{
Analyse der Ergebnisse und Komplikationen der ureterorenoskopischen Steintherapie von 1998 bis 2008 unter Berücksichtigung des verwendeten Instrumentariums sowie der Steincharakteristika
}

\author{
INAUGURAL-DISSERTATION \\ zur Erlangung des Doktorgrades \\ der Medizinischen Fakultät \\ der Georg-August-Universität zu Göttingen
}

\author{
vorgelegt von \\ Philipp-Paul Lumma \\ aus \\ Paderborn
}

Göttingen 2010 
D e k a n:

I. Berichterstatter:

II. Berichterstatter/in:

III. Berichterstatter/in:
Prof. Dr. med. C. Frömmel

PD Dr. med. H. Loertzer

PD Dr. med. C. Dumont

Tag der mündlichen Prüfung: $\quad$ 02. Mai 2011 


\section{Inhaltsverzeichnis}

1 Einleitung 1

1.1 Therapie der Harnleitersteine . . . . . . . . . . . . . . . . . . . . . . 1

1.1.1 Konservative Therapie und Chemolitholyse . . . . . . . . . . . 2

1.1 .2 ESWL . . . . . . . . . . . . . . . . . . 4

$1.1 .3 \mathrm{PCNL} \ldots \ldots \ldots \ldots \ldots$

1.1 .4 Offene und laparoskopische Steinsanierung . . . . . . . . . . . 6

1.1 .5 URS . . . . . . . . . . . . . . . . . . 7

1.1.5.1 Geschichte der URS . . . . . . . . . . . . . . 7

1.1.5.2 Indikationen . . . . . . . . . . . . . . . 8

1.1.5.3 Kontraindikationen . . . . . . . . . . . . . . . 10

1.1.5.4 Technik . . . . . . . . . . . . . . . . . . . 10

1.1.5.5 Ergebnisse der URS . . . . . . . . . . . . . . . . . 11

1.1.5.6 Komplikationen der URS . . . . . . . . . . . . . . 11

1.2 Fragestellung . . . . . . . . . . . . . . . . . . 13

2 Material, Patienten und Methoden $\quad 14$

2.1 Ausgewertete Untersuchungen . . . . . . . . . . . . . . . . . . . . . . . 14

2.2 Patienten . . . . . . . . . . . . . . . . . . . . . . . . 14

2.2.1 Alter und Geschlecht . . . . . . . . . . . . . . . . . . . . . . . . 15

2.2 .2 Vorerkrankungen . . . . . . . . . . . . . . . . . 15

2.3 Operativer Eingriff . . . . . . . . . . . . . . . . . . . . . . . . 17

2.3.1 Vorgehen . . . . . . . . . . . . . . . . . 17

2.3.2 Verwendete Geräte . . . . . . . . . . . . . . . . . . . . . . . . . . 19

2.3.2.1 Ureterorenoskope ... . . . . . . . . . . . . . 19

2.3.2.2 Instrumente . . . . . . . . . . . . . . . . . 20

3 Ergebnisse $\quad 22$

3.1 Harnsteine . . . . . . . . . . . . . . . . . . . . . . . 22

3.1 .1 Steinposition . . . . . . . . . . . . . . . . 22

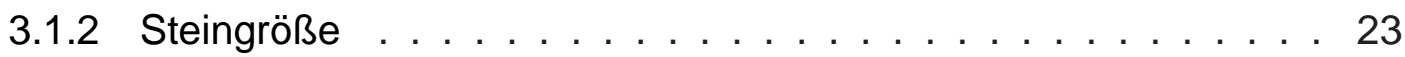


3.1 .3 Steinanalyse . . . . . . . . . . . . . . . . . . . 24

3.2 Behandlung . . . . . . . . . . . . . . . . . 26

3.2 .1 Steinfreiheit . . . . . . . . . . . . . . . . . . 27

3.2 .2 Komplikationen . . . . . . . . . . . . . . . . . . . . . . 34

4 Diskussion 42

5 Zusammenfassung $\quad 56$

6 Literaturverzeichnis $\quad 58$ 


\section{Abkürzungsverzeichnis}

$\begin{array}{ll}\text { BMI } & \begin{array}{l}\text { Body-Mass-Index } \\ \text { Ch }\end{array} \\ \text { Charrière, Durchmesser für urologische Geräte, Katheter } \\ \text { und Endoskope. } 1 \mathrm{Ch}=1 \text { French = 1/3 Millimeter } \\ \text { DJ } & \begin{array}{l}\text { Doppel-J-Ureterschiene } \\ \text { distaler Ureter }\end{array} \\ \text { DU } & \text { Extrakorporale Stoßwellenlithotripsie } \\ \text { KM } & \text { Kontrastmittel } \\ \text { Kompl. } & \text { Komplikation/en } \\ \text { MU } & \text { mittlerer Ureter } \\ \text { NBKS } & \text { Nierenbeckenkelchsystem } \\ \text { Pat. } & \text { Patient/en } \\ \text { PCNL } & \text { Perkutane Nephrolitholapaxie } \\ \text { PU } & \text { proximaler Ureter } \\ \text { UMG } & \text { Universitätsmedizin Göttingen } \\ \text { URS } & \text { Ureterorenoskopie }\end{array}$




\section{Einleitung}

Die Behandlung von Harnsteinen ist auch heute noch ein wichtiger Teilbereich der Urologie. In Deutschland steigt seit vielen Jahren die Neuerkrankungsrate der Urolithiasis bei gleichzeitig hoher Prävalenz. Hesse et al. (2003) stellten in einer großen epidemiologischen Studie fest, dass die Prävalenzrate von 4\% im Jahr 1979 auf 4,7\% im Jahr 2000 angestiegen war. Die Inzidenz der Urolithiasis hatte sich von 0,54\% 1979 auf $1,47 \%$ im Jahr 2000 fast verdreifacht.

Die Ursachen dieser Entwicklung scheinen vielfältig zu sein. Es wird angenommen, dass die verbesserte medizinische Grundversorgung zu einer häufigeren Diagnosestellung und Behandlung führt. Veränderte Lebens- und Ernährungsgewohnheiten werden als kausale Gründe für ein vermehrtes Auftreten von Steinerkrankungen diskutiert (Knoll et al. 2009 a).

Die Therapie der Urolithiasis hat sich seit Anfang der 1980er Jahre deutlich verändert. Kam zuvor für viele Harnsteine häufig nur eine offene chirurgische Operation in Betracht, sind seitdem endoskopische Verfahren wie die URS und PCNL und nicht invasive Verfahren wie die ESWL entwickelt und erprobt worden. Die offene Chirurgie des Nierensteins war bis zur praktischen Einsetzbarkeit der neuen Systeme die einzige Möglichkeit zur Steinbehandlung, aktuell macht sie laut Knoll et al. (2009 a) nur noch einen Anteil von etwa $1 \%$ aus.

Die URS wurde durch neue und bessere Ureterorenoskope und Lithotripsiesysteme sowie durch eine wachsende Erfahrung der Urologen mit dieser Technik zunehmend bedeutender. Fortschritte im Bereich der flexiblen Ureterorenoskopie führten dazu, dass kleine Harnsteine $<1 \mathrm{~cm}$ erfolgreich auch im Nierenbecken und in den Nierenkelchen behandelt werden konnten. Neben ESWL und PCNL gehört mittlerweile auch die URS im Bereich des Hohlsystems zu den effektiven Behandlungsmethoden der Urolithiasis. Im proximalen Harnleiter ist je nach Indikation (s. Abbildung 1, Seite 9) die ESWL bevorzugte Behandlungsmethode, während die URS im distalen Ureter bevorzugt eingesetzt wird.

\subsection{Therapie der Harnleitersteine}

Patienten mit Harnleitersteinen stellen sich in der Regel mit akuten unilateralen Schmerzen vor, die durch den Übertritt des Harnsteins in den Ureter hervorgerufen werden. 
Die Schmerzen variieren in vielen Fällen in ihrer Intensität und strahlen zum Teil bis in die Leiste ein. Weiterhin findet sich häufig ein klopfschmerzhaftes Nierenlager und eine verminderte Darmmotilität. Blut und Entzündungszeichen im Urin vervollständigen das klinische Bild. Im Gegensatz zu den Harnleitersteinen können Nierensteine asymptomatisch bleiben und im Rahmen von Folgeschädigungen der Niere oder zufällig entdeckt werden.

Ist ein Harnstein diagnostiziert, stehen in der Regel mehrere Behandlungsoptionen zur Verfügung, um diesen erfolgreich zu therapieren. Die Wahl der Methode hängt dabei wesentlich von den Faktoren 'Steinlokalisation', 'Steingröße' und 'verfügbare Behandlungszeit' ab. Die vermutete oder bekannte Steinzusammensetzung und die Wahrscheinlichkeit von Komplikationen fließen ebenfalls in die Therapieentscheidung mit ein (Knoll et al. 2009 a).

Von Bedeutung für das therapeutische Vorgehen ist weiterhin die Schmerzsymptomatik, vorhandene Komorbiditäten, zurückliegende Steinereignisse, Allergien, Medikamenteneinnahme, die Familienanamnese, Schwangerschaft und auch die persönliche Situation des Patienten und seine Erwartungen an die Behandlung. Die Einschätzung der „Spontanabgangsfähigkeit“ und die vermutliche Dauer bis zum spontanen Abgang des Steins können einen konservativen Therapieversuch ermöglichen oder ausschlieBen.

\subsubsection{Konservative Therapie und Chemolitholyse}

Die konservative Therapie bietet die Möglichkeit, den Spontanabgang unter klinischer Kontrolle und medikamentöser Therapie zu begleiten. Wird ein Harnstein als spontan abgangsfähig angesehen, kommt diese Behandlungsmethode in Frage. Vor allem kleine $(<4 \mathrm{~mm})$ und distal im Harntrakt gelegene Steine sind für die Behandlung geeignet. Falls keine akuten Komplikationen drohen, und der Patient bereit ist, die nicht vorhersagbare Dauer bis zum Steinabgang abzuwarten, kann ein konservativer Therapieversuch erfolgen. Für die Dauer der Behandlung finden regelmäßig Kontrolluntersuchungen statt, um mögliche Komplikationen frühzeitig zu erkennen. Der Patient wird angewiesen, seinen Urin zu sieben, um abgegangene Harnsteine zu bemerken und einer Analyse zuzuführen. Nach dem Steinabgang wird die Steinfreiheit dokumentiert. Die konservative Therapie kann medikamentös unterstützt werden. Die wichtigsten Einsatzfelder bestehen in der Förderung des Spontanabgangs durch alpha- Rezepto- 
renblocker oder Ca-Antagonisten. Die Wahrscheinlichkeit eines Spontanabgangs kann mit diesen Medikamenten um 65\% erhöht werden (Hollingsworth et al. 2006). Auch weitere Substanzen wie Antibiotika, Steroide, Spasmoanalgetika, Nitrate und nichtsteroidale Antiphlogistika können den Steinabgang unterstützen (Knoll et al. 2009 a).

Abzugrenzen ist die konservative Therapie vom beobachtenden Abwarten "watchful waiting“. Hier wird ein asymptomatischer Harnstein (meist Nierenstein), von dem keine Gesundheitsgefährdung ausgeht, durch regelmäßige Kontrollen überwacht.

Eine weitere Therapiemöglichkeit bietet die Chemolitholyse. Die orale Chemolitholyse bewirkt durch die Einnahme von harnalkalisierenden Substanzen wie Alkalicitraten oder Natriumbicarbonat eine Auflösung oder Verkleinerung von Harnsäuresteinen. Dies gelingt durch die Erhöhung des Urin-pH auf Werte zwischen 7,0 und 7,2. Ergänzend kann durch die Einnahme von Allopurinol die Bildung von Harnsäure vermindert werden. Cystinsteine sind ebenfalls durch eine Harnalkalisierung behandelbar. Kontraindiziert ist die orale Chemolitholyse bei bestehendem Harnwegsinfekt. Bei einer nachgewiesenen Niereninsuffizienz ist die Gabe von Alkalicitraten kontraindiziert. Generell sollte auf eine ausreichende Diurese während der Behandlung geachtet werden.

Wenn die orale Litholyse und andere Behandlungsverfahren nicht möglich waren oder erfolglos blieben, kann ein Behandlungsversuch mit der lokalen Chemolitholyse (Irrigationschemolitholyse) erfolgen. Bei diesem Verfahren werden die Harnsteine mit einem Wirkstoff umspült, was einen suffizienten Hin- und Abtransport der Substanz bis zum Stein voraussetzt und nur bei Harnsäure-, Cystin- und Infektsteinen (außer Ammoniumuratsteinen) zum Erfolg führen kann. Eingesetzt werden beim Harnsäurestein Natriumbikarbonat oder THAM-Lösung (Trihydroxymethyl-aminomethan). Nach vorheriger Lithotripsie können auch Struvitsteine aus der Gruppe der Infektsteine durch eine lokale Litholyse effektiv behandelt werden. Die lokale Chemolitholyse setzt einen invasiven Eingriff durch die Haut oder retrograd durch den Ureter zur Einbringung der benötigten Spülkatheter voraus und dauert einige Tage bis zu mehreren Wochen. Durch die größere Invasivität im Vergleich zur oralen Litholyse sind spezielle Komplikationen möglich. Dazu gehören toxische oder septische Einschwemmungen durch die Spülkatheter.

Vorteil der konservativen Therapie und der oralen Chemolitholyse ist die fehlende oder geringe Invasivität der Behandlung. Bei Harnsäuresteinen bietet die orale Chemolitholyse eine komplikationsarme und wirksame Therapieoption (Knoll et al. 2009 a). Nachteile bestehen in der teils wochenlangen Behandlungszeit, die bis zu einem Steinab- 
gang vergehen kann. In dieser Zeit ist auf eine medikamentöse Schmerzbehandlung oft nicht zu verzichten. Bei unklarem Erfolg ist die Bereitschaft des Patienten, den Ausgang abzuwarten, auch von der persönlichen Einstellung abhängig.

Kontraindiziert ist eine abwartende Behandlung bei ausgeprägter Harnstauung, stummer Niere oder infizierter Harnstauungsniere. In diesen Fällen ist eine unmittelbare Intervention nötig. Durch eine perkutane oder transurethrale Drainage kann die gestaute Niere entlastet werden. Zusätzlich erfolgt bei Infektion eine antibiotische Therapie.

Die Wahrscheinlichkeit, dass ein Harnstein spontan abgeht, ist vor allem von der GröBe und der Lokalisation abhängig. Je kleiner der Harnstein ist und je weiter distal dieser liegt, umso höher ist die Spontanabgangsfähigkeit. Diese liegt bei Steinen $\leq 5$ $\mathrm{mm}$ bei $68 \%$ und bei Steinen $>5 \mathrm{~mm}$ und $\leq 10 \mathrm{~mm}$ bei $47 \%$ (Preminger et al. 2007). Mit zunehmender Steingröße verlängert sich die Zeitspanne bis zum Spontanabgang. Steine bis $2 \mathrm{~mm}$ Durchmesser brauchen durchschnittlich 1 Woche, Steine bis $3 \mathrm{~mm} 2$ Wochen und Steine von 4-6 mm durchschnittlich 3 Wochen bis zum Spontanabgang. Aus diesen Gründen sollten nur Steine bis $4 \mathrm{~mm}$ Durchmesser als spontan abgangsfähig angesehen werden (Knoll et al. 2009 a).

\subsubsection{Extrakorporale Stoßwellenlithotripsie (ESWL)}

Durch elektromagnetische, piezoelektrische oder elektrohydraulische Generatoren werden akustische Wellen erzeugt, die durch Fokussierung zu Stoßwellen werden. Die vom ESWL-Gerät abgegebene Stoßwelle durchläuft ein Medium, welches in seinen akustischen Eigenschaften dem menschlichen Gewebe ähneln sollte. Trifft die Stoßwelle auf den Harnstein, wirkt dieser der Ausbreitung der Welle entgegen und es kommt zur Energieübertragung. Je nach Steinart variiert die resultierende Zerstörung des Kristallgefüges.

Die ESWL findet im proximalen Bereich des Harnleiters und im Bereich des Nierenbeckens und der Nierenkelche bei geringer Steinlast die häufigste Anwendung und stellte lange das etablierte Verfahren für proximale Steine dar. Vorteile der Behandlung ergeben sich aus der geringen Invasivität. Eine Narkose ist fakultativ möglich, aber im Gegensatz zu anderen invasiven Behandlungen nicht nötig. Es treten wenige bedrohliche Komplikationen auf. Die Inzidenz des renalen/perirenalen Hämatoms als eine der gravierendsten Komplikationen ist mit unter $1 \%$ Inzidenz gering (Knoll et al. 2009 a). 
Neben der akuten Blutungsneigung, bestehender Antikoagulation und dem unbehandelten Harnwegsinfekt existieren weitere Kontraindikationen und damit mehr als bei der URS. Dazu zählen die Schwangerschaft, Tumoren im behandelten Bereich, das Aortenaneursyma, die akute Pankreatitis und der unbehandelte Hypertonus. Für Träger von Herzschrittmachern und Defibrillatoren ergeben sich gerätespezische Einschränkungen. Um eine ESWL möglich zu machen, muss der Harnstein zu orten sein. Dies ist bei bestimmten Steinzusammensetzungen (z. B. Harnsäuresteinen) röntgenologisch nur eingeschränkt zu erreichen. Es besteht jedoch die Möglichkeit, „röntgennegative" Harnsteine durch eine Kontrastmittelgabe anhand auftretender Kontrastmittelaussparungen zu lokalisieren. Auch bei sehr adipösen Erwachsenen sowie Kleinkindern kann die Steinortung erschwert sein. Die Ortung durch die Sonografie ist nicht überall möglich und bleibt vor allem auf das Nierenbecken beschränkt. Bei vielen Patienten ist der Spontanabgang von Fragmenten nach der ESWL behindert. Gründe hierfür ist eine Steinlokalisation in den Nierenkelchen (besonders im unteren Kelch), eine große Steinmasse, Stenosen des harnleitenden Systems, Divertikel und anatomische Anomalien (Knoll et al. 2009 b). Um Komplikationen durch den Abgang der Fragmente entgegenzuwirken, kann eine Ureterschiene eingelegt werden.

\subsubsection{Perkutane Nephrolitholapaxie (PCNL)}

Zu Beginn des Eingriffs erfolgt die Einlage eines Ureterkatheters, dessen Spitze unter Bildgebung kurz vor dem Nierenbeckenabgang platziert wird. Der Katheter erschwert die spätere Abschwemmung von Steinfragmenten. Es kann alternativ auch ein Okklusionskatheter verwendet werden, der im Hohlsystem verankert wird. Das Kelchsystem kann vor der transkutanen Punktion über den Katheter mit Flüssigkeit dilatiert oder mit Kontrastmittel gefüllt werden. Bei nicht ausreichender Dilatation des Hohlsystems ist eine erfolgreiche Anlage der perkutanen Nephrostomie erschwert. Die Punktion erfolgt unter sonographischer und eventuell zusätzlicher radiologischer Kontrolle. Der Zugang zum Harnstein erfolgt wegen eines geringen Blutungsrisikos meist durch eine Verbindung zum dorsalen Kelch der unteren Kelchgruppe, aber auch andere Zugänge sind möglich und zum Teil nötig. Durch die Bougierung des Stichkanals kann das Nephroskop mit einem Durchmesser zwischen 16 Ch und 30 Ch (5,3 mm - 10 $\mathrm{mm}$ ) im Anschluss bis in den punktierten Bereich vorgeschoben werden. Der Einsatz eines flexiblen Ureterorenoskops mit einem geringeren Durchmesser ist bei schwierigen Verhältnissen möglich. Zur Steinentfernung dienen Instrumente, die auch bei der 
Ureterorenoskopie Verwendung finden. Nach Abschluss der Steinbehandlung wird ein Nephrostomiekatheter zur Harnableitung und Ermöglichung eines eventuell notwendigen Zweiteingriffs in den Punktionskanal eingelegt.

Die PCNL ist bei großen Steinen $>2 \mathrm{~cm}$ im Nierenbecken, der oberen und mittleren Kelchgruppe sowie Ausgusssteinen Therapiemethode der ersten Wahl (Knoll et al. 2009 a). Oft ist es die letzte Möglichkeit, bei großen Steinformationen Steinfreiheit zu erreichen.

Als Kontraindikationen gelten die bestehende Antikoagulation, der unbehandelte Harnwegsinfekt, die akute Blutungsneigung, die Schwangerschaft und Tumoren im Operationsbereich. Teils schwere Komplikationen sind möglich. Dazu zählen transfusionsbedürftige Blutungen, Nierenverlust, Darmperforation, Nierenbeckenperforation, das „Einschwemmungs-Syndrom“ (TUR-Syndrom) und postoperative Infekte. Bei bestehenden Nieren- oder Skelettanomalien, Darm- oder Pleurainterpositionen kann der Eingriff erschwert oder nicht möglich sein. Eine Nephrostomieeinlage wird empfohlen, bis der Harnabfluss sowie die Stein- und Blutungsfreiheit gesichert ist.

\subsubsection{Offene und laparoskopische Steinsanierung}

Die offene und laparoskopische Steinentfernung wird heutzutage nur noch selten eingesetzt. Offene Steinentfernungen machen mittlerweile nur noch etwa 1\% der Harnsteinbehandlungen im Bereich der Niere aus (Knoll et al. 2009 a). Meist wird bei der offenen Steinentfernung ein Flankenschnitt subkostal durchgeführt. Der Zugang zum Harnstein kann durch das Gewebe der Niere (transparenchymatös) erfolgen oder bevorzugt durch eine das Nierengewebe schonende Pyelolithotomie. Eine Nierenteilresektion ist bei vorgeschädigtem Gewebe möglich.

Die offene und in zunehmendem Maße laparoskopische Steinbehandlung ist indiziert, wenn alle anderen therapeutischen Methoden entweder nicht möglich sind, oder keine guten Erfolgsaussichten versprechen. Dies ist vor allem bei einer sehr großen Steinmenge, dilatierten Kelchen mit engen Kelchhälsen und großer Steinmenge in verschiedenen Kelchen der Fall. Besteht neben einer manifesten Uro- bzw. Nephrolithiasis zusätzlich Bedarf zu einer chirurgischen Korrektur im Bereich der Niere, gilt die offene und laparoskopische Steinsanierung ebenfalls als indiziert. Kontraindikationen sind die akute Blutungsneigung, die bestehende Antikoagulation und der unbehandelte Harnwegsinfekt. Bei adipösen Patienten, nach Voroperationen und bei Vorliegen 
von anatomischen Anomalien ist die Behandlung erschwert.

\subsubsection{Ureterorenoskopie (URS)}

Die URS liefert hohe Steinfreiheitsraten zwischen 84-94\% (Preminger et al. 2007) und minimiert damit Wiederholungsbehandlungen. Diese liegen bei distalen Harnleitersteinen zwischen 0-4\%, im Vergleich zu 10-21\% bei der ESWL (Knoll et al. 2009 a). Die Ureterorenoskopie wird als eine sehr gute therapeutische und diagnostische Behandlungsmethode in der Urologie angesehen, die nur eine geringe Komplikationsrate aufweist (Geavlete et al. 2006). Es muss im Gegensatz zur PCNL und der offenen sowie laparoskopischen Therapie nicht geschnitten werden. Für die Steinbehandlung ist bis auf Einzelfälle eine Narkose nötig.

1.1.5.1 Geschichte der URS Die erste Ureterorenoskopie wurde 1912 mit einem Zystoskop an einem 2 Monate alten Säugling durchgeführt. Bei massiv dilatiertem Harnleiter gelang es, das Gerät bis zum Nierenbecken vorzuschieben. Die routinemäBige Anwendung der Ureterorenoskopie erfolgte erst ab den späten 1970er Jahren (Conlin et al. 1997).

1977 wurden mehrere erfolgreiche URS zur Behandlung von distalen Harnleitertumoren unabhängig voneinander beschrieben. Die Harnleiterspiegelung wurde mit Kinderzystoskopen durchgeführt, mit denen der distale Ureter allerdings nur kurzstreckig darstellbar war (Goodman 1977). Die Erfindung der Stablinsenoptik sorgte für eine bessere intramurale Sicht der Geräte (Fuchs 2006). 1980 erfolgte der Bau eines langen Ureterorenoskops für Erwachsene, das bis zum Nierenbecken einsetzbar war (PerezCastro Ellendt et al. 1984). Nun war auch die Behandlung von großen Steinen bis in den proximalen Harntrakt unter Sicht möglich. Nachteilig bei diesen starren Ureterorenoskopen mit Linsensystem war die Entstehung einer „halbmondförmigen“ Sichteinschränkung bei Biegung. Ein Nachteil, den Geräte zunehmend nicht mehr aufwiesen, die sich ab 1986 durchsetzten (Lamskemper 2006). Die URS wurde mit zunehmender technischer Verbesserung häufiger zur Therapie von Harnsteinen eingesetzt und löste andere Behandlungsmethoden ab. Der häufigere Einsatz der Ureterorenoskopie förderte gleichzeitig die Entwicklung neuer Lithotripsiesysteme.

Bei der Steindesintegration verhinderten kaliberstarke Ultraschallsonden noch bis Ende der 1980er Jahre eine Lithotripsie unter Sicht. Harnsteine wurden mit dem Körb- 
chen gefangen und mussten blind lithotripsiert werden. Mit der Beschreibung der Farbstofflaserlithotripsie (Dretler et al. 1987) und der Einführung von dünneren Sonden wurde der Eingriff wesentlich erleichtert. Durch die Anwendung von Fieberoptiken konnten dünnere semirigide Ureterorenoskope entwickelt werden. Bei einem Spitzenumfang der semirigiden Geräte von 7-8 Ch (2,33 mm - 2,66 mm) war eine Bougierung des Ureterostiums oft nicht mehr nötig. Dies entspricht den heutigen Ureterorenoskopen.

Die Entwicklung des flexiblen Ureterorenoskops begann in den 1960er Jahren. Da aber zu Beginn Spül- und Arbeitskanäle fehlten und damit der Einsatz der flexiblen Geräte sehr eingeschränkt war, stand das semirigide Ureterorenoskop lange im Vordergrund. Ende der 1980er Jahre wurde erstmals von Patientenserien berichtet, die erfolgreich mit flexiblen Ureterorenoskopen behandelt wurden (Abdel-Razzak und Bagley 1992). Durch die Einführung von dünneren flexiblen Ureterorenoskopen mit verhältnismäßig großen Arbeitskanälen um 3,5 Ch $(1,16 \mathrm{~mm})$ wurde die Entwicklung weiter vorangetrieben (Grasso und Bagley 1994). Die effektive Steinbehandlung und Lithotripsie mit flexiblen Geräten hat durch die Einführung des Ho:YAG (Holmium: YttriumAluminium-Garnet)-Lasers weitere Fortschritte gemacht.

Verbesserte, noch dünnere Ureterorenoskope, weiterentwickelte Lithotripsiesysteme und eine größere Erfahrung der Urologen mit dieser Technik haben zu einer zunehmenden Aufwertung der URS im Vergleich zur ESWL geführt. Die technische Weiterentwicklung zu einer besseren Haltbarkeit und Benutzerfreundlichkeit konnte vor allem bei den flexiblen Geräten beobachtet werden. Neuerungen wie die Ureterorenoskopie mit digitaler Bildverarbeitung ("Chip-on-the-Tip" Technologie) könnten diese Entwicklung fortsetzen (Knoll und Alken 2006).

1.1.5.2 Indikationen Als Indikation für die URS gelten therapieresistente Koliken, erfolglose Vorbehandlungen (ESWL, Zeiss'sche Schlinge), große Harnleitersteine mit unwahrscheinlichem Spontanabgang, die Steinstraße nach ESWL mit Beschwerden sowie der zunehmende Harnstau (Nierenbeckenkelchektasie).

Die aktuellen „S2-Leitlinien zur Diagnostik, Therapie und Metaphylaxe der Urolithiasis" empfehlen als ersten Schritt bei Vorliegen eines Harnsteins, die Möglichkeit der konservativen Therapie zu prüfen. Nur wenn der Spontanabgang nicht abgewartet werden kann, soll eine weiteres Vorgehen nach entwickelten Algorithmen erfolgen (s. Abbildung 1). 
In dieser Arbeit sind drei dieser Algorithmen für die Behandlung des Harnleitersteins, Nierenbeckensteins und Steine der oberen, mittleren und unteren Kelchgruppe angeführt. Weitere Algorithmen und Informationen zu speziellen Situationen sind in dem ausführlichen Leitlinientext publiziert (Knoll et al. 2009 a).

Abbildung 1: Algorithmen zur Steinbehandlung im Ureter und Nierenbeckenkelchsystem aus den „S2-Leitlinien zur Diagnostik, Therapie und Metaphylaxe der Urolithiasis“" (Knoll et al. 2009 b).

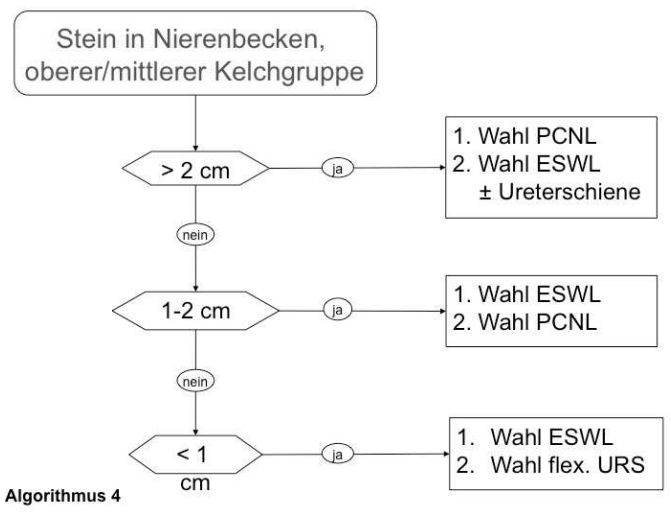

(a) Algorithmus Nierenbeckenstein, oberer und mittlerer Kelchstein, S. 918

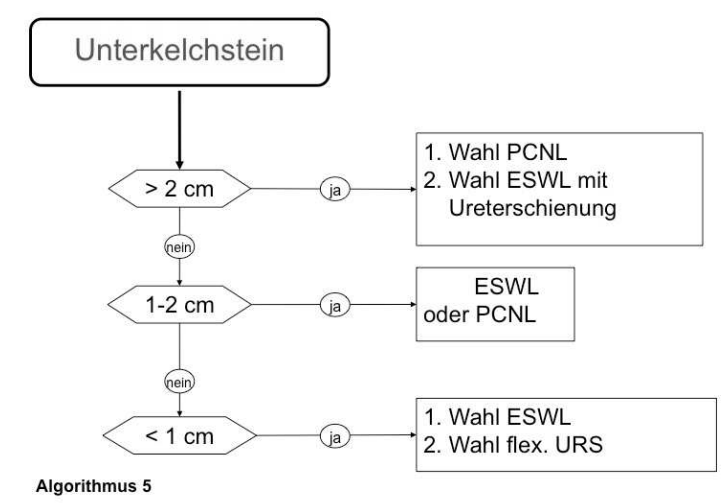

(b) Algorithmus Unterkelchstein, S. 918

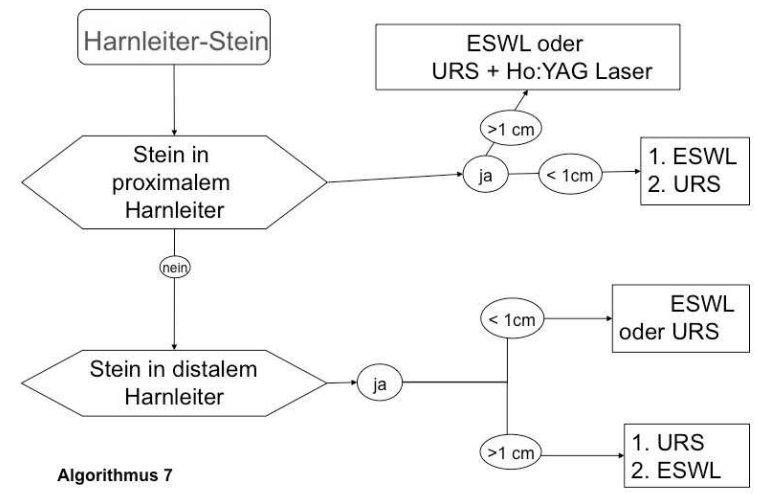

(c) Algorithmus Harnleiterstein, S. 920

Für die Behandlung des Harnsäuresteins ergeben sich von Abbildung 1 abweichende Therapieempfehlungen. Wesentlicher Bestandteil der Therapie ist der Versuch der oralen Chemolitholyse. Kann eine alleinige konservative Therapie nicht abgewartet werden oder war diese nicht erfolgreich, ist die orale Litholyse mit anderen Behandlungsverfahren (ESWL, PCNL) kombinierbar. 
1.1.5.3 Kontraindikationen Kontraindikationen der URS sind der unbehandelte Harnwegsinfekt, die akute Blutungsneigung, sowie die bestehende Antikoagulation. Als relative Kontraindikationen gilt die Schwangerschaft.

Die URS ist erschwert, wenn der Zugang zum Stein über den Harnleiter behindert ist. Dies ist beispielsweise bei einem Prostataadenom, Harnleiterstrikturen und einer neu angelegten Harnableitung (Conduit u.a.) der Fall.

1.1.5.4 Technik Mit dünnen semirigiden Geräten kann nach transurethraler Einführung das Ureterostium oft ohne Bougierung intubiert werden. Falls eine Aufweitung nötig ist, stehen Ballon- und Kunststoffbougies zur Verfügung. Diese können über einem Führungsdraht oder direkt in den Ureter vorgeschoben werden. Häufig wird bei einer akuten Kolik zur Entlastung bereits eine Ureterschiene eingelegt. Schließt sich nun innerhalb weniger Tage die URS zur Steinbehandlung an, ist eine intraoperative Bougierung in der Regel nicht mehr nötig.

Bei flexiblen Geräten erleichtert die Einlage eines Führungsdrahtes unter Sicht häufig das Vorschieben des Ureterorenoskops im Ureter. Möglich ist es ebenfalls, eine Einführhülse (Zugangsschaft) einzusetzen, welche unter Zuhilfenahme eines vorher eingelegten Drahtes positioniert wird. Ist die Hülse ausgerichtet, lässt sich das flexible Gerät durch den inneren Hohlraum der Einführhülse vorschieben.

Zur Steinextraktion stehen verschiedene Instrumente zur Verfügung. Mit der Steinfasszange und dem Dormiakörbchen ist eine Extraktion des kompletten Steins in toto möglich. Dies gelingt meist bis zu einer Steingröße von 2-3 mm. Auch die Bergung von Steinfragmenten nach Lithotripsie kann mit den beiden Instrumenten erfolgen. Als Lithotripsiesysteme zur intramuralen Desintegration von Harnsteinen stehen etwa stoßwellenbasierte Systeme zur Verfügung. Dazu gehören Laser (v.a. Ho:YAG-Laser), aber auch elektrohydraulische Lithotriptoren. Beide Systeme können im Gegensatz zu mechanischen Lithotriptoren auch in dünnen, flexiblen Ureterorenoskopen angewendet werden. Mechanische Lithotripsiesysteme erzeugen außerhalb des Körpers eine Energie, die auf den Stein fortgeleitet wird. Dazu gehören Systeme, die Ultraschallwellen nutzen, pneumatisch-ballistische (Lithoclast ${ }^{\circledR}$ ) und elektrokinetisch-ballistische Systeme (Lamskemper 2006).

Elektrohydraulische Lithotripsiesysteme sollten aufgrund der hohen Perforationsgefahr nicht mehr verwendet werden (Knoll et al. 2009 a). Mit pneumatisch-ballistischen Systemen (Lithoclast ${ }^{\circledR}$ ) wurden zwar bei gleichzeitiger guter Lithotripsieleistung von 
porösen Steinen nur wenige unkalkulierte Harnleiterverletzungen beobachtet, doch besteht in der Fortbewegung des Harnsteins (Propulsion) während der Lithotripsie ein Nachteil (Sözen et al. 2003). Bei Ultraschall-basierten Lithotriptern wird durch eine simultane Absaugung kleinstes Steinmaterial unmittelbar entfernt, was sich vorteilhaft auf die intramurale Sicht auswirkt. Der Holmium:Yttrium-Aluminium-Garnet (Ho:YAG)Laser gilt wegen seiner Eigenschaft, alle vorkommenden Steine effektiv zu desintegrieren, der Einsetzbarkeit in semirigiden und flexiblen Geräten sowie der geringen Komplikationsrate als Goldstandard für die intrakorporale Lithotripsie (Knoll et al. 2009 a).

Am Ende der Operation kann ein Ureterkatheter zur Sicherung des Harnabflusses eingelegt werden. Erforderlich ist die Harnleiterschienung bei multiplen Restfragmenten, nach langer Operationszeit, bei ödematösem Steinbett und nach Harnleiterirritation (Dilatation, Perforation, Schleimhautarrosion). Abhängig von der Indikation (Harnleiterperforation, Mukosaödem etc.) sollte die Liegedauer der Ureterschiene angepasst werden. Häufig wird die Schiene für 5-14 Tage im Ureter belassen. Eine routinemäßige Schienung des Ureters nach atraumatischer URS ist nicht erforderlich (Knoll et al. 2009 a, Preminger et al. 2007, Gerber und Stockton 2006).

1.1.5.5 Ergebnisse der URS Technische Innovationen haben die Erfolgsraten der URS über die Zeit verbessert. Aktuelle Publikationen spielen daher zur Beurteilung der momentanen Effektivität der Untersuchung eine besondere Rolle.

Preminger et al. (2007) publizierten die Steinfreiheitsraten von 9218 ureterorenoskopisch bei Urolithiasis behandelten Patienten in Abhängigkeit von der Steinlokalisation. Diese lagen zwischen $81 \%$ und 94\%. Pearle et al. (2005) werteten die Erfolgsraten bei der Behandlung von Unterkelchsteinen mit dem flexiblen Ureterorenoskop aus, s. Tabelle 1.

1.1.5.6 Komplikationen der URS Die Anzahl schwerwiegender Früh- und Spätkomplikationen bei der Ureterorenoskopie ist gering. Gravierende Komplikationen wie Sepsis, Harnleiterperforation und Harnleiterabriss traten in 3-11\% der Behandlungen auf (Knoll et al. 2009 a). Harnleiterstrikturen werden zunehmend seltener beobachtet. Tabelle 2 zeigt publizierte Komplikationsereignisse.

Obwohl durch die Einlage einer Doppel-J-Ureterschiene eine Ureterperforation meist komplett ausheilt, ist bei sehr langstreckigen Perforationen eine offene oder laparo- 
Tabelle 1: Ergebnisse der URS in der Literatur

\begin{tabular}{lcc}
\hline Preminger et al. (2007) (Metaanalyse) & & \\
\cline { 1 - 2 } Lokalisation & Pat./Studiengruppen & Steinfreiheitsrate \\
\hline proximaler Ureter & $2242 / 46$ & $81 \%$ \\
mittlerer Ureter & $1024 / 30$ & $86 \%$ \\
distaler Ureter & $5952 / 59$ & $94 \%$ \\
\hline Pearle et al. (2005) & & \\
\cline { 1 - 1 } untere Kelchgruppe & $35 / 1$ & $50 \%$ \\
\hline
\end{tabular}

Tabelle 2: Komplikationen der URS in der Literatur

\begin{tabular}{llcc}
\hline \multicolumn{2}{l}{ Preminger et al. (2007) (Metaanalyse) } & & \\
\cline { 1 - 2 } Komplikation & Lokalisation & Pat./Studiengruppen & Komplikationsrate \\
\hline Sepsis & proximaler Ureter & $360 / 8$ & $4 \%$ \\
& mittlerer Ureter & $199 / 4$ & $4 \%$ \\
& distaler Ureter & $1954 / 7$ & $2 \%$ \\
Ureterstriktur & proximaler Ureter & $987 / 8$ & $2 \%$ \\
& mittlerer Ureter & $326 / 7$ & $4 \%$ \\
& distaler Ureter & $1911 / 16$ & $1 \%$ \\
Ureterverletzung & proximaler Ureter & $1005 / 10$ & $6 \%$ \\
& mittlerer Ureter & $514 / 10$ & $6 \%$ \\
& distaler Ureter & $4529 / 23$ & $3 \%$ \\
Harnwegsinfekt & proximaler Ureter & $224 / 2$ & $4 \%$ \\
& mittlerer Ureter & $63 / 1$ & $2 \%$ \\
& distaler Ureter & $458 / 3$ & $4 \%$ \\
\hline Geavlete et al. (2006) & & \\
\hline Ureterstriktur & ganzer Ureter & $2735 / 1$ & $0,1 \%$ \\
Ureterperforation & ganzer Ureter & $2735 / 1$ & $0,7 \%$ \\
Ureterabriss & ganzer Ureter & $2735 / 1$ & $1,1 \%$ \\
Fieber/Sepsis & ganzer Ureter & $2735 / 1$ & \\
\hline
\end{tabular}

skopische Operation teilweise notwendig. Kommt es intraoperativ zu einem Harnleiterabriss, besteht die Indikation zur sofortigen offenen oder laparoskopischen Operation. 


\subsection{Fragestellung}

In dieser Arbeit sollen retrospektiv die Ergebnisse und Komplikationen der ureterorenoskopischen Steinbehandlung in der Fachabteilung Urologie des Universitätsklinikums Göttingen untersucht werden.

Der Einfluss semirigider und flexibler Ureterorenoskope sowie der zur Steinbehandlung verwendeten Instrumente auf den Erfolg der Behandlung soll genauer betrachtet werden.

Mögliche Einflussfaktoren wie etwa Lokalisation und Größe der Harnsteine auf die erreichte Steinfreiheitsrate sollen ausgewertet werden.

Die Behandlungsresultate sollen mit den bereits publizierten Ergebnissen anderer Einrichtungen verglichen werden, um eine Aussage über die Qualität und Effektivität der Steintherapie treffen zu können. 


\section{Material, Patienten und Methoden}

\subsection{Ausgewertete Untersuchungen}

Im ausgewerteten Zeitraum 01.01.1998 bis 31.12.2008 wurden 805 Patienten in der Fachabteilung Urologie des Universitätsklinikums Göttingen ureterorenoskopisch behandelt. Ein symptomatischer Harnstein des Ureters oder Nierenbeckenkelchsystems wurde in 594 Fällen therapiert. Bei 46 Patienten konnte intraoperativ bei Verdacht auf Harnleiterstein kein Nachweis eines Konkrements erbracht werden. Es wurde bei vorheriger Symptomatik ein Spontanabgang angenommen. Bei 144 Patienten erfolgte die URS aus diagnostischen Gründen, etwa der Gewinnung von Biopsien. Bei 21 Patienten wurde eine Harnleiterstenose durch Einsatz des Lasers behandelt.

\subsection{Patienten}

Zur Auswertung kamen 594 Steinbehandlungen von insgesamt 509 Patienten. 12,4\% der Patienten wurden mehr als einmal an einem Harnstein behandelt. Die genaue Aufteilung der Behandlungshäufigkeit zeigt Abbildung 2.

Abbildung 2: Behandlungshäufigkeit pro Patient (594 Behandlungen / 509 Patienten)

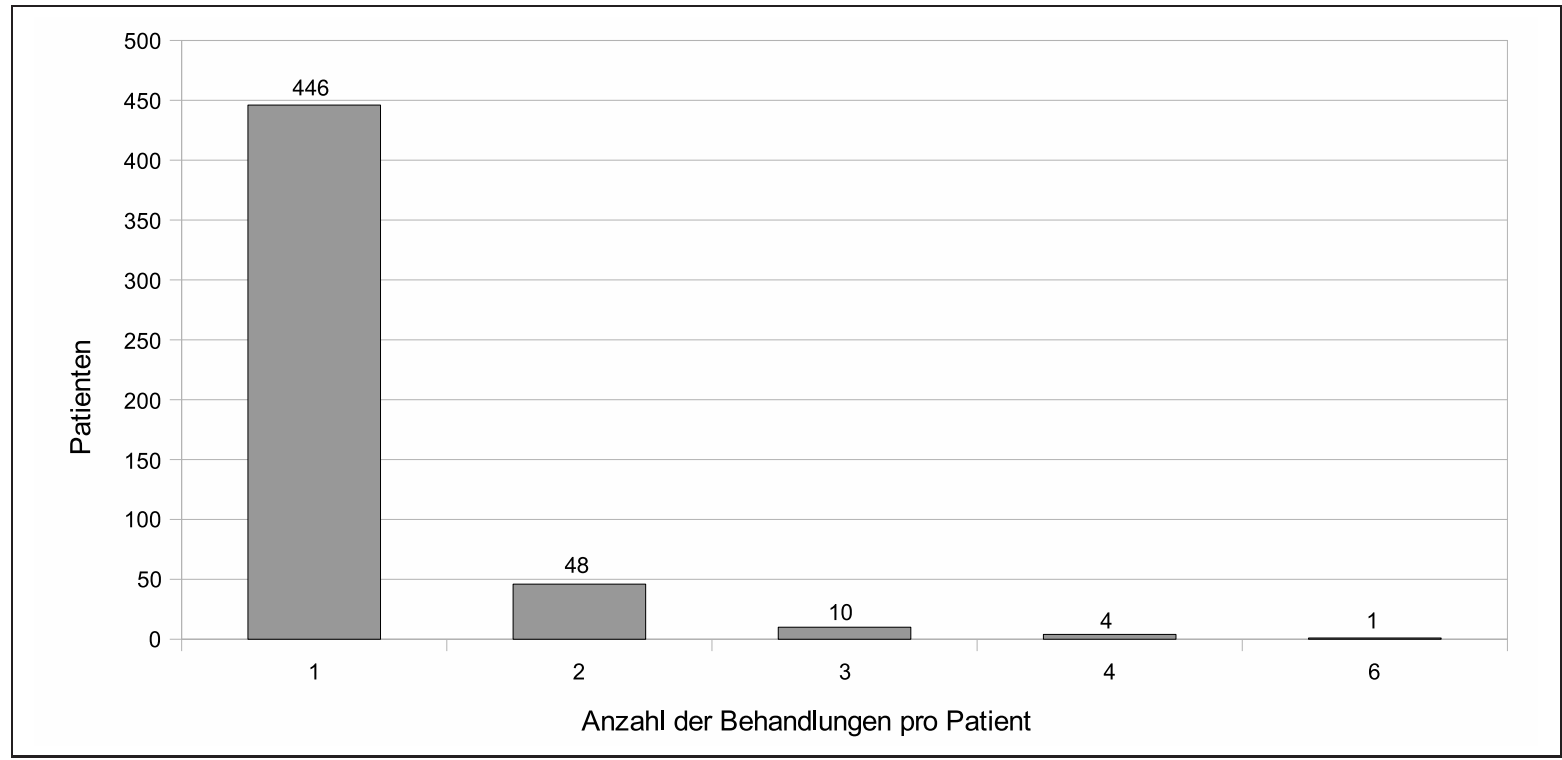




\subsubsection{Alter und Geschlecht}

Das Alter der Patienten lag zum Zeitpunkt des ureterorenoskopischen Ersteingriffs zwischen 7 und 84 Jahren. Der Mittelwert betrug 49,8 Jahre, der Median 49,7 Jahre.

Es wurden 160 (31,4\%) Frauen und 349 (68,6\%) Männer behandelt.

Abbildung 3: Alter der Patienten bei der ersten Steinbehandlung ( $n=509)$

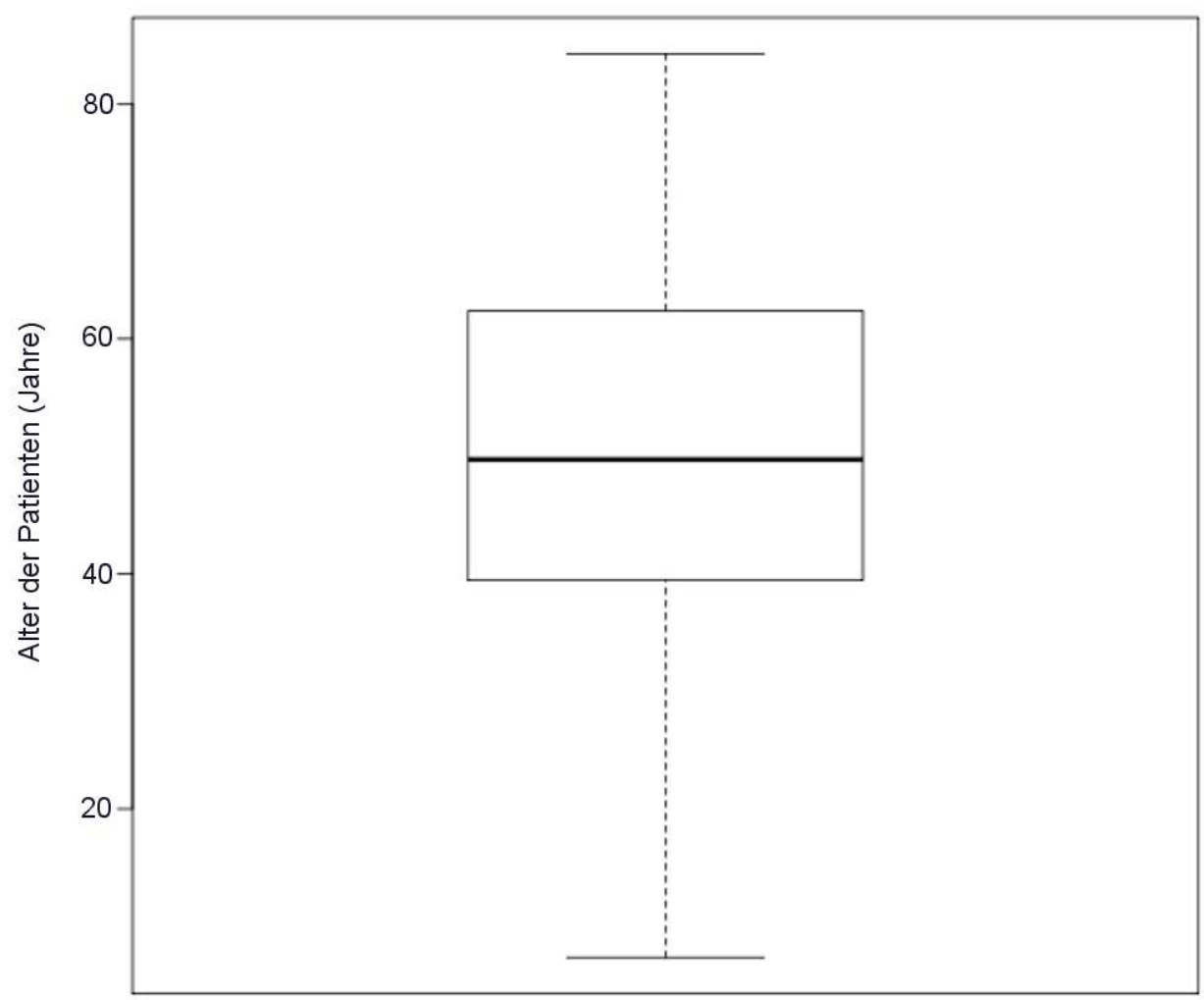

Abbildung 3 zeigt die statistische Verteilung des Patientenalters zum Zeitpunkt der ersten Steinbehandlung.

\subsubsection{Vorerkrankungen}

Die Erfassung der Vorerkrankungen erfolgte anhand der Pflegekurve sowie der Krankenakte, die während des stationären Aufenthalts der Patienten geführt wurden. Durch die Erfassung von Größe und Gewicht der Patienten, war die Berechnung des BodyMass-Index (BMI) möglich (World Health Organization 2004). Die Vorerkrankungen Diabetes mellitus, Hypertonus sowie Hyperlipoproteinämie wurden anhand der verabreichten Medikamente bestimmt. Bei Mehrfachbehandlungen eines Patienten wurden 
ausschließlich die beim ersten Aufenthalt bestehenden Erkrankungen in die Auswertung einbezogen.

Eine Berechnung des BMI war bei 486 von 509 Patienten möglich. Für die Gesamtheit der Patienten ergab sich ein Mittelwert von $27,2 \mathrm{~kg} / \mathrm{m}^{2}$ und ein Median von $26,8 \mathrm{~kg} / \mathrm{m}^{2}$. Der BMI wurde minimal mit $12,4 \mathrm{~kg} / \mathrm{m}^{2}$ und maximal mit $57,1 \mathrm{~kg} / \mathrm{m}^{2}$ bestimmt. Die für beide Geschlechter berechneten Werte zeigt Tabelle 3.

Tabelle 3: Body-Mass-Index und Geschlechteraufteilung

\begin{tabular}{llll}
\hline Männer $(\mathrm{n}=336)$ & \multicolumn{3}{l}{ Frauen $(\mathrm{n}=150)$} \\
\hline Wert & BMI & Wert & BMI \\
\hline Mittelwert & 27,5 & Mittelwert & 26,6 \\
Median & 27,0 & Median & 26,3 \\
Maximum & 57,1 & Maximum & 42,5 \\
Minimum & 12,4 & Minimum & 15,6 \\
\hline
\end{tabular}

Bei Anwendung der Body-Mass-Index-Gewichtsklassifikation für Erwachsene (World Health Organization 2004) fallen 10 Patienten unter 18 Jahren aus der Betrachtung. Weiter erfolgte in Tabelle 4 eine Auftrennung der Patienten in Männer und Frauen, um unterschiedlichen Gewichtsnormbereichen Rechnung zu tragen.

Tabelle 4: Body-Mass-Index und Geschlechteraufteilung (nach WHO Klassifikation)

\begin{tabular}{lrrrlrrr}
\hline \multicolumn{2}{l}{ Männer $\geq 18$ Jahre $(\mathrm{n}=331)$} & \multicolumn{5}{l}{ Frauen $\geq 18$ Jahre $(\mathrm{n}=145)$} \\
\hline Gewicht & BMI & Pat. & Anteil & Gewicht & BMI & Pat. & Anteil \\
\hline Untergewicht & $<18,5$ & 4 & $1,2 \%$ & Untergewicht & $<18,5$ & 6 & $4,1 \%$ \\
Normgewicht & $18,5-24,9$ & 99 & $29,9 \%$ & Normgewicht & $18,5-24,9$ & 50 & $34,5 \%$ \\
Übergewicht & $25-30$ & 141 & $42,6 \%$ & Übergewicht & $25-30$ & 48 & $33,1 \%$ \\
Adipositas & $>30$ & 87 & $26,3 \%$ & Adipositas & $>30$ & 41 & $28,3 \%$ \\
\hline
\end{tabular}

Ein medikamentös behandelter oder vorbeschriebener Hypertonus lag bei 123 der 509 Patienten $(24,2 \%)$ vor.

Eine medikamentös behandelte oder vorbeschriebene Hyperlipoproteinämie wurde bei 37 der 509 Patienten (7,3\%) gefunden.

Ein Diabetes mellitus wurde bei Einnahme eines oralen Antidiabetikums, der Verabfolgung von Insulin oder bei vorbeschriebener Diagnose als vorhanden angesehen. Dies war bei 49 der 509 Patienten (9,6\%) der Fall. 


\subsection{Operativer Eingriff}

Dem Eingriff ging im Universitätsklinikum Göttingen regelhaft eine Blutuntersuchung voraus. Bestimmt wurden ein kleines Blutbild, Leberwerte, Blutzucker, Elektrolyte, Kreatinin und Gerinnungsparameter.

Bei Patienten mit vorbekanntem Steinleiden und erneutem Nachweis von röntgendichten Konkrementen wurde in aller Regel ein Calcium- und Phosphatspiegel sowie das Parathormon zum Ausschluß eines Hyperparathyreoidismus bestimmt.

Eine präoperativ durchgeführte Röntgendiagnostik machte es oft möglich, Konkremente zu erkennen und ihre Lokalisation im Harntrakt zu beschreiben. Anhand der Steinlokalisation wurde geplant, ob die Ureterorenoskopie primär eher mit dem semirigiden oder einem flexiblen Ureterorenoskop durchgeführt werden sollte.

Aufgeklärt wurde über die Möglichkeit der konsekutiven Schienenanlage, der möglichen Notwendigkeit einer offenen Operation bei eventuellen Komplikationen, der antibiotischen Therapie zur Behandlung einer Harnwegsinfektion, weiterhin bestehender Schmerzen, Strikturen des Ureters sowie der Notwendigkeit einer erneuten Operation. Komplikationen im Rahmen der Anästhesie (Punktion der Gefäße und Medikamentenverabreichung) wurden ebenfalls genannt.

Nach Indikationsstellung zur Steinsanierung, Aufklärung und Voruntersuchung, erfolgte der Eingriff mit Hilfe des Ureterorenoskops.

\subsubsection{Vorgehen}

Die Patienten wurden zur Vorbereitung auf die Operation anästhesiert und in Steinschnittlage gelagert. Als Narkoseformen kamen Intubationsnarkose oder Spinalanästhesie zur Anwendung. Alle Patienten wurden während des operativen Eingriffs durch einen Anästhesisten und eine Anästhesiepflegekraft betreut und überwacht.

Die Lagerung des Patienten erfolgte in Steinschnittlage auf dem Durchleuchtungstisch „Uroskop D“ von Siemens. Es handelte sich um eine digitale Röntgenanlage mit Bildspeicherfunktion.

Vor Beginn des Eingriffs erfolgte eine sterile Abdeckung und die Desinfektion des äuBeren Genitales.

Primär durchgeführt wurde eine Zystoskopie mit einem 21 Ch $(7 \mathrm{~mm}$ ) Zystoskop (Uni- 
versalendoskop) der Firma Olympus oder einem semirigiden URS-Gerät zur diagnostischen Blasenspiegelung zum Ausschluß eines malignen Geschehens im Blasenbereich und zur Entfernung einer eventuell präoperativ eingelegten Harnleiterschiene. Es folgte die Intubation des Harnleiterostiums, ggf. nach Vorlegen eines Drahtes und Ausspiegeln des Harnleiters von distal nach proximal bis ins Nierenbecken bzw. bis zum Konkrement. Bei Harnleiterkonkrementen wurden diese über das semirigide Instrument entfernt (s. Abbildung 4), bei Vorliegen von Nierenkelch- bzw. Nierenbeckenkonkrementen erfolgte ggf. der Einsatz des flexiblen Ureterorenoskops. Die Behandlung mit dem flexiblen Gerät wurde durch die Positionierung eines Führungsdrahts vorbereitet, welcher als Leitschiene für eine Einführhülse (Führungsschaft) mit einem Außendurchmesser von $13 \mathrm{Ch}(4,3 \mathrm{~mm})$ diente. Durch den inneren Kanal der Hülse konnte nun das flexible Ureterorenoskop bis vor die ermittelte Steinposition vorgeschoben werden, um dort eine Extraktion des Steins bzw. eine Lithotripsie durchzuführen.

Am Ende der Operation wurde eine diagnostische Spiegelung des betroffenen Harnleiters bis zum Beginn des Nierenbeckens durchgeführt und dokumentiert.

In Abhängigkeit vom intraoperativen Ergebnis und eventuellen Läsionen des Urogenitaltraktes erfolgte die Einlage einer Doppel-J-Ureterschiene und ggf. eines transurethralen Katheters. Dies vorrangig um mögliche Komplikationen durch die Sicherstellung einer ausreichenden Harndrainage zu vermeiden und die Entlastung des Urogenitaltraktes zu erreichen.

Abbildung 4: Endoskopische Betrachtung einer Steinbehandlung. Quelle: Privatarchiv K.-D. Plothe (), UMG

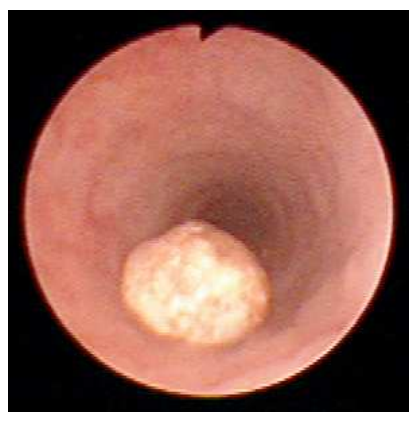

(a) Harnstein im Ureter

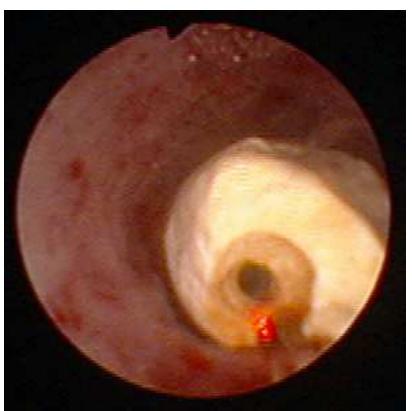

(b) Lithotripsie mit dem Ho:YAG-Laser

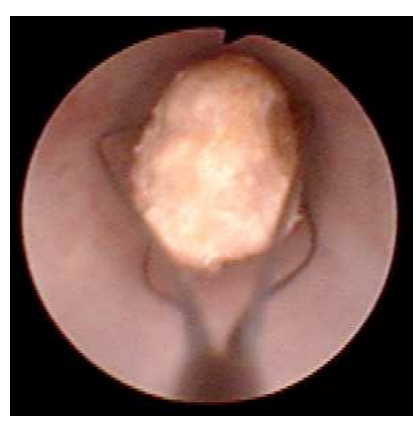

(c) Extraktion mit dem Dormiakörbchen

Als obligatorische Untersuchung im weiteren Verlauf der Behandlung erfolgte eine sonographische Untersuchung spätestens am Tag nach der Operation. Ein Labor mit kleinem Blutbild, Elektrolyten und Kreatininwert wurde ebenfalls grundsätzlich be- 
stimmt. Zur weiteren Kontrolle diente regelmäßig eine Röntgenaufnahme ohne Kontrastmittel der betroffenen Seite zum Ausschluss verbliebener Konkremente.

\subsubsection{Verwendete Geräte}

Die Untersuchungen wurden im betrachteten Zeitraum mit semirigiden und flexiblen Ureterorenoskopen durchgeführt. Bei den semirigiden Geräten fanden zwei Ureterorenoskope Anwendung, die sich im Durchmesser der Einführ- und Spitzenteile unterschieden. Beide Geräte wurden getrennt voneinander in Bezug auf Steinfreiheit und Komplikationen ausgewertet. Im Bereich der flexiblen Ureterorenoskope kamen drei verschiedene Modelle zum Einsatz. Diese wurden unter dem Überbegriff „flexibles Ureterorenoskop“ zusammengefasst und nicht getrennt ausgewertet.

Die durch den Arbeitskanal der Ureterorenoskope geführten Instrumente dienten der Lithotripsie bzw. der Entfernung der Steine aus dem Ureter. Eingesetzt wurden mechanische Steinfasszangen, das Nitinolkörbchen (Dormiakörbchen), der Holmium:YttriumAluminium-Garnet (Ho:YAG)-Laser, die mechanisch (pneumatisch-ballistisch) arbeitende Sonotrode sowie die Zeiss'sche Schlinge. Die beiden letztgenannten Instrumente waren für den Einsatz in den verwendeten flexiblen Ureterorenoskopen nicht geeignet.

2.3.2.1 Ureterorenoskope Im gesamten Zeitraum der Untersuchung wurden zwei verschiedene semirigide Ureterorenoskope der Reihe „OES Pro“ der Firma Olympus eingesetzt. Das kaliberstärkere Gerät verjüngte sich stufenförmig von 9,8 Ch $(3,27$ $\mathrm{mm})$ auf 8,6 Ch $(2,87 \mathrm{~mm})$ und verfügte über einen 6,4 Ch (2,13 mm) Arbeitskanal, eine 7 Grad Optik und war $43 \mathrm{~cm}$ lang. Das dünnere semirigide Ureterorenoskop war in seiner Optik und Länge identisch, verjüngte sich jedoch von 7,8 Ch (2,6 mm) auf 6,4 Ch (2,13 mm) und hatte einen Arbeitskanaldurchmesser von 4,2 Ch (1,4 mm). In Abbildung 5 ist das große semirigide Gerät zu sehen.

Es wurden verschiedene flexible Ureterorenoskope genutzt. Zu Beginn der Untersuchung wurde das Modell „URF-P4“ der Firma Olympus eingesetzt, welches durch das Nachfolgemodell „URF-P5“ mit einem Durchmesser des Einführteils von 8,4 Ch $(2,8$ $\mathrm{mm})$, einem Spitzendurchmesser von 5,3 Ch $(1,77 \mathrm{~mm})$ und einem Durchmesser des Arbeitskanals von 3,6 Ch (1,2 mm) abgelöst wurde. Das „URF-P5“ war 180 Grad aufwärts und 275 Grad abwärts beweglich, bei einer Arbeitslänge von 70 cm. Ab 2002 
Abbildung 5: Arbeitsmaterialien für die URS. Quelle: Privatarchiv K.-D. Plothe @ , UMG

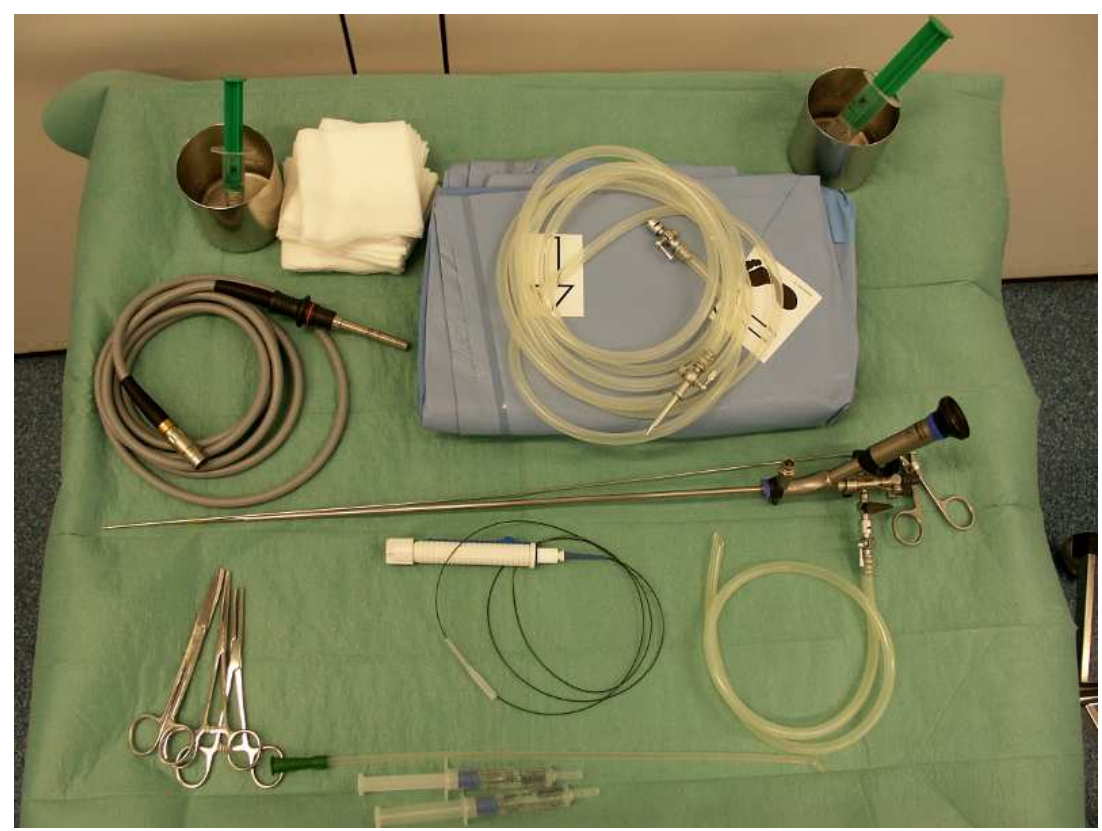

wurde das „DUR-8 Elite“ der Firma ACMI (später Olympus) mit einem Schaft- und Spitzendurchmesser von 8,7 Ch $(2,9 \mathrm{~mm})$ und einem Arbeitskanaldurchmesser von 3,6 Ch (1,2 mm) eingesetzt. Das Gerät war um 130 Grad abwärts beweglich und hatte eine Arbeitslänge von $64 \mathrm{~cm}$.

Um das intraoperative Vorschieben und Positionieren der flexiblen Ureterorenoskope zu erleichtern, wurde in Göttingen eine Einführhülse der Firma Boston Scientific verwendet. Die $46 \mathrm{~cm}$ lange Hülse "Navigator"TM" verfügte über einen Außendurchmesser von $13 \mathrm{Ch}$ (4,3 mm). Abbildung 6 zeigt, wie ein flexibles Ureterorenoskop über die gelegte Einführhülse vorgeschoben wird.

2.3.2.2 Instrumente Über den gesamten Untersuchungszeitraum dieser Arbeit hinweg kamen mechanische Steinfasszangen und das Dormiakörbchen als Extraktionsgeräte zum Einsatz. Die Zeiss' sche Schlinge wurde bis 1999 verwendet. Zur Lithotripsie dienten der Holmium: Yttrium-Aluminium-Garnet (Ho:YAG)-Laser und die von 1998 bis 2002 eingesetzte Sonotrode. Abbildung 7 zeigt die beiden am häufigsten eingesetzten Instrumente in dieser Arbeit.

Zur Harnleiterschienung wurden Doppel-J-Ureterschienen unterschiedlichen Durchmessers verwendet. 
Abbildung 6: Einführhülse Boston Scientific "Navigator'TM". Quelle: Privatarchiv K.-D. Plothe (C), UMG

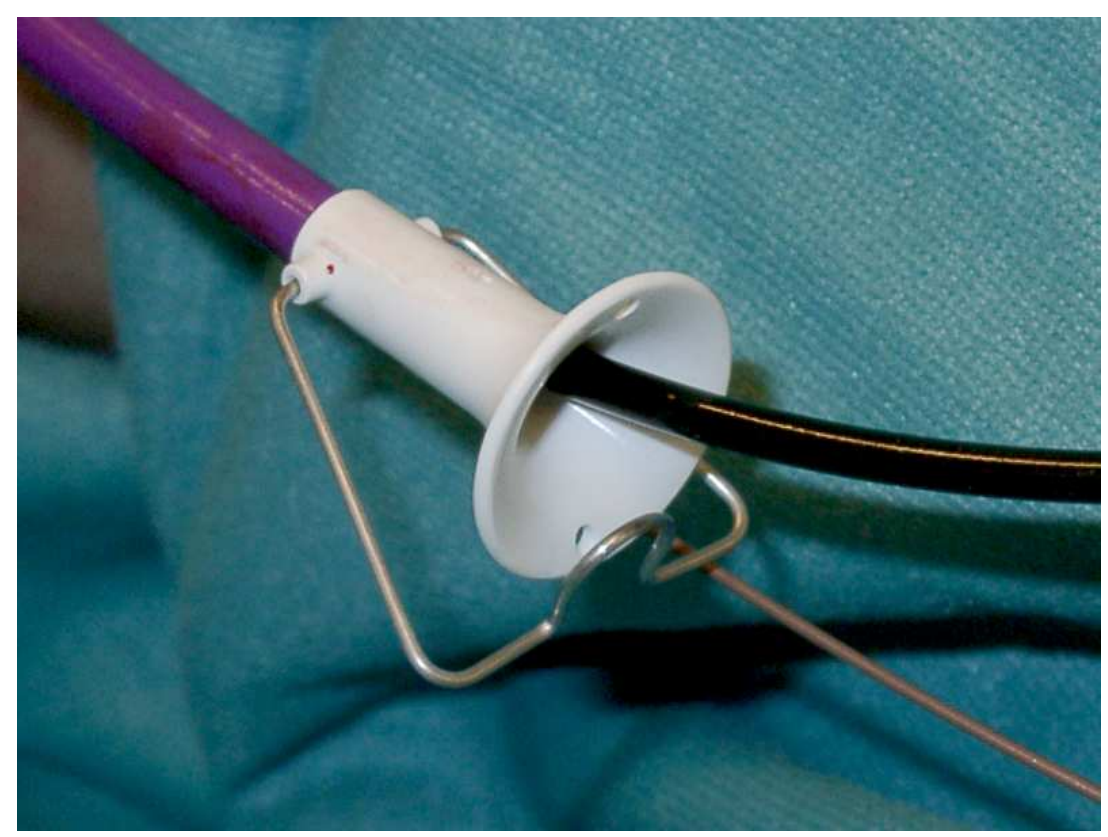

Abbildung 7: Körbchen und Ho:YAG Laserfaser im Arbeitskanal eines flexiblen Ureterorenoskops. Quelle: Privatarchiv K.-D. Plothe (C), UMG

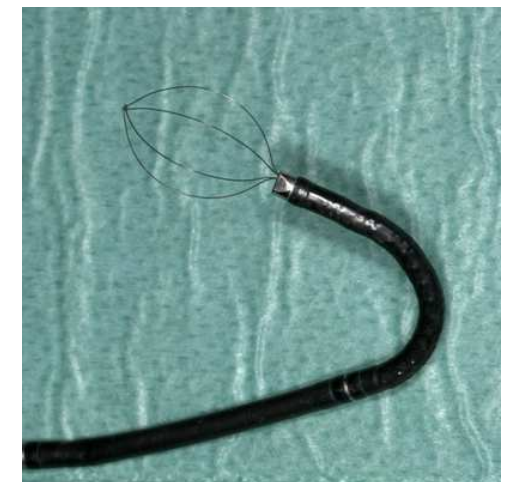

(a) aufgespanntes Dormiakörbchen

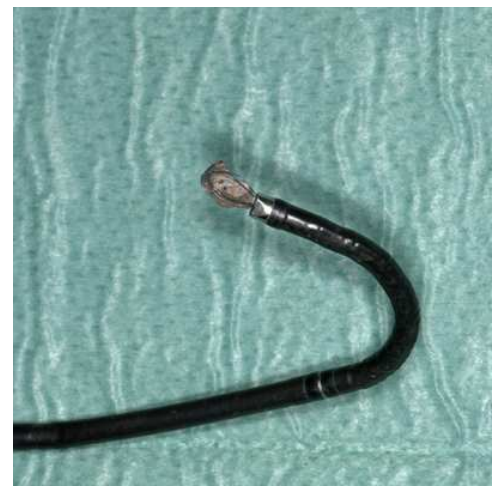

(b) Dormiakörbchen mit Harnstein

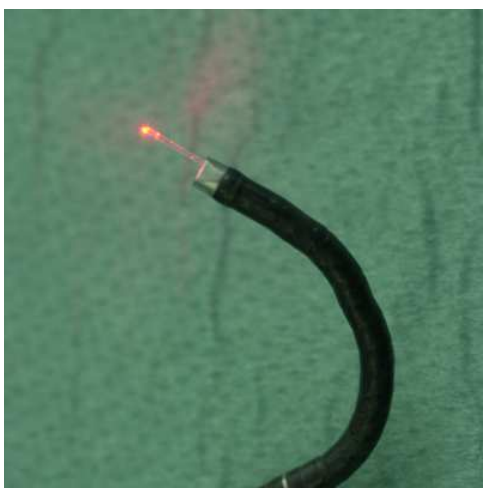

(c) Ho:YAG Laserfaser 


\section{Ergebnisse}

In dieser Studie wurde eine Ureterorenoskopie nur dann als erfolgreich gewertet, wenn die komplette Steinfreiheit im Sinne der Entfernung sämtlicher sichtbarer Konkremente erfolgt war. Verblieben Konkremente im Harnleiter oder Nierenbeckenkelchsystem, selbst wenn diese spontan abgangsfähig erschienen, so galt die Behandlung als nicht erfolgreich. Des Weiteren wurde eine URS nur dann als erfolgreich angesehen, wenn sich keine weiteren auxiliären Maßnahmen wie etwa ESWL oder PCNL auf das Steingebiet anschlossen. Die Aussage über die Steinfreiheit bezieht sich grundsätzlich auf den behandelten Harnstein. In seltenen Fällen wurde ein symptomatischer Harnleiterstein behandelt, während ein therapeutisch mit der URS nicht behandelbarer Stein (meist im Nierenbecken oder Kelchbereich) dort belassen wurde. Der Erfolg der Behandlung bezieht sich in einem solchen Fall auf den Harnstein, für den die URS als Behandlungsmöglichkeit indiziert war.

\subsection{Harnsteine}

Die behandelten Harnsteine wurden anhand ihrer Lokalisation im Harntrakt, ihrer maximalen Kantenlänge und chemischen Beschaffenheit gruppiert und beschrieben.

\subsubsection{Steinposition}

Um die Steinposition beschreiben zu können, wurde der Harnleiter mit angrenzendem Hohlsystem in dieser Studie in vier Abschnitte eingeteilt. Den ersten Abschnitt bildet das Nierenbeckenkelchsystem, welches sich bis zum pyeloureteralen Übergang erstreckt. Der Ureter wird in dieser Arbeit in einen proximalen, mittleren und distalen Abschnitt unterteilt. Der mittlere Abschnitt beschreibt den Verlauf des Ureters in Knochendeckung des Beckens. Dies ist der Bereich zwischen Promontorium und einer gedachten Linie durch die Querfortsätze des Lendenwirbelkörpers 4 und die Oberkanten der Beckenschaufeln. Proximaler und distaler Ureter grenzen daran an. Der intramurale Teil des Ureters wird dem distalen Abschnitt zugerechnet.

Im untersuchten Zeitraum wurden 594 Steinbehandlungen durchgeführt. Davon verteilten sich 286 Behandlungen (48,1\%) auf die rechte Seite und 308 Behandlungen $(51,9 \%)$ auf die linke Seite. 
Es wurden bei 594 Behandlungen insgesamt 696 in unterschiedlichen Abschnitten des Harntrakts gelegene Harnsteine therapiert. Bei 497 Patienten (83,7\%) war der Harnstein in einem Abschnitt des Harntrakts nachweisbar. Bei 92 Patienten (15,5\%) wurden Steine in zwei und bei 5 Patienten $(0,8 \%)$ in drei Abschnitten gefunden.

Bei 46 weiteren Untersuchungen konnte trotz entsprechender Symptomatik (Urinbefund, Klinik) während der URS kein Stein mehr nachgewiesen werden. Dies wurde als „unbemerkter Spontanabgang“ gewertet.

Die Höhenverteilung der Harnsteine wird in Tabelle 5 dargestellt.

Tabelle 5: Steinverteilung und Steinlokalisation im Harntrakt (696 Steine)

\begin{tabular}{lcclcr}
\hline \multicolumn{2}{l}{ in einem } & Abschnitt $(\mathrm{n}=497)$ & & \multicolumn{3}{l}{ in mehreren Abschnitten $(\mathrm{n}=199)$} \\
\hline Höhe & Anzahl & Anteil & Höhe & Anzahl & Anteil \\
\hline NBKS & 115 & $23,1 \%$ & NBKS & 77 & $38,7 \%$ \\
PU & 75 & $15,1 \%$ & PU & 41 & $20,6 \%$ \\
MU & 88 & $17,7 \%$ & MU & 35 & $17,6 \%$ \\
DU & 219 & $44,1 \%$ & DU & 46 & $23,1 \%$ \\
\hline
\end{tabular}

\subsubsection{Steingröße}

Bei 594 behandelten Patienten konnte der Harnstein in 568 (95,6\%) Fällen komplett ausgemessen werden. Dies erfolgte bei schattengebenden Steinen durch die Vermessung des Steinschattens im Röntgenbild. Bei nicht schattengebenden Steinen konnte oftmals ein Urogramm zur Bestimmung der Steingröße herangezogen werden. Bei 26 Patienten (4,4\%) war die Harnsteingröße nicht zu klären. Tabelle 6 gibt die errechneten Werte für die Gesamtheit der vermessenen Harnsteine wieder.

Tabelle 6: Steingröße

\begin{tabular}{lcr}
\hline Statistik & max. Kantenlänge $(\mathrm{mm})$ & $\mathrm{mm}^{2}$ \\
\hline Mittelwert & 5,4 & 22,9 \\
Median & 5,0 & 17,5 \\
Maximum & 18,0 & 225,0 \\
Minimum & 1,5 & 1,5 \\
\hline
\end{tabular}

Es erfolgte eine Einteilung der 568 Steine in drei Gruppen anhand der maximal gemessenen Kantenlänge. 331 Steine (58,3\%) waren $\leq 5 \mathrm{~mm}, 212$ Steine $(37,3 \%)$ waren $>5$ 
$\mathrm{mm}$ und $\leq 10 \mathrm{~mm}$ und 25 Steine $(4,4 \%)$ waren $>10 \mathrm{~mm}$. Bei 26 Patienten konnte die Steingröße nicht bestimmt werden.

\subsubsection{Steinanalyse}

Die Steinanalysen wurden zu Beginn dieser Studie von Januar 1998 bis Dezember 2000 im Labor der Asklepios Klinik in Pasewalk und im Anschluss bis April 2008 im Labor des Albert-Schweitzer-Krankenhauses in Northeim durchgeführt. Von April 2008 bis zum Zeitpunkt der letzten hier eingeflossenen Steinanalyse im Dezember 2008 erfolgte die Bestimmung im Labor des „Medizinischen Versorgungszentrums Wagner Stibbe“ in Göttingen.

Es wurden 298 Steine von 509 Patienten (58,6\%) ausgewertet. Wurden bei einem Patienten durch Mehrfachbehandlungen mehrere Steine gewonnen, so wurde nur der erste analysierte Stein in die Untersuchung einbezogen.

Nicht alle präoperativ vermessenen Steine wurden analysiert. Gründe hierfür sind etwa ein Scheitern der Operation, zu kleine Fragmente nach Lithotripsie, Mitnahme des Steins durch den Patienten und Probleme bei der Archivierung des nach Patientenentlassung eingetroffenen Analyseergebnisses.

Es zeigte sich, dass auf 298 Harnsteine 194 Monosteine (65,1\%) und 104 Mischsteine $(34,9 \%)$ entfielen. Die Mischsteine ließen sich weiter in 98 Steine mit zwei Komponenten und 6 Steine mit drei Komponenten untergliedern (s. Abbildung 8).

Bei den Monosteinen zeigte sich, dass 70,1\% (136) Steine aus Calciummonooxalat (Whewellit) bestanden. Sieben weitere Komponenten machten zusammen lediglich einen Anteil von 29,9\% aus. Abbildung 9 zeigt die Zusammensetzung der analysierten monomineralischen Harnsteine.

Aus den 104 analysierten Mischsteinen konnten 214 Komponenten isoliert werden (98 mal zwei Komponenten, 6 mal drei Komponenten).

Die einzelnen Komponenten (s. Abbildung 10) machten unabhängig von der Häufigkeit, mit der sie in den Steinen nachweisbar waren, einen unterschiedlich großen prozentualen Anteil an der nachgewiesenen Menge von Harnsteinsubstanz aus.

Steine aus Struvit (Magnesiumammoniumphosphat-Hexahydrat), Carbonatapatit (Calciumphosphat) und Ammoniumhydrogenurat machen die Gruppe der sogenannten „Infektsteine“ aus. Meist zeigt sich ein Infekt mit Urease-bildenden Keimen, der zu 
Abbildung 8: Verteilung der Steinkomponenten $(\mathrm{n}=298)$

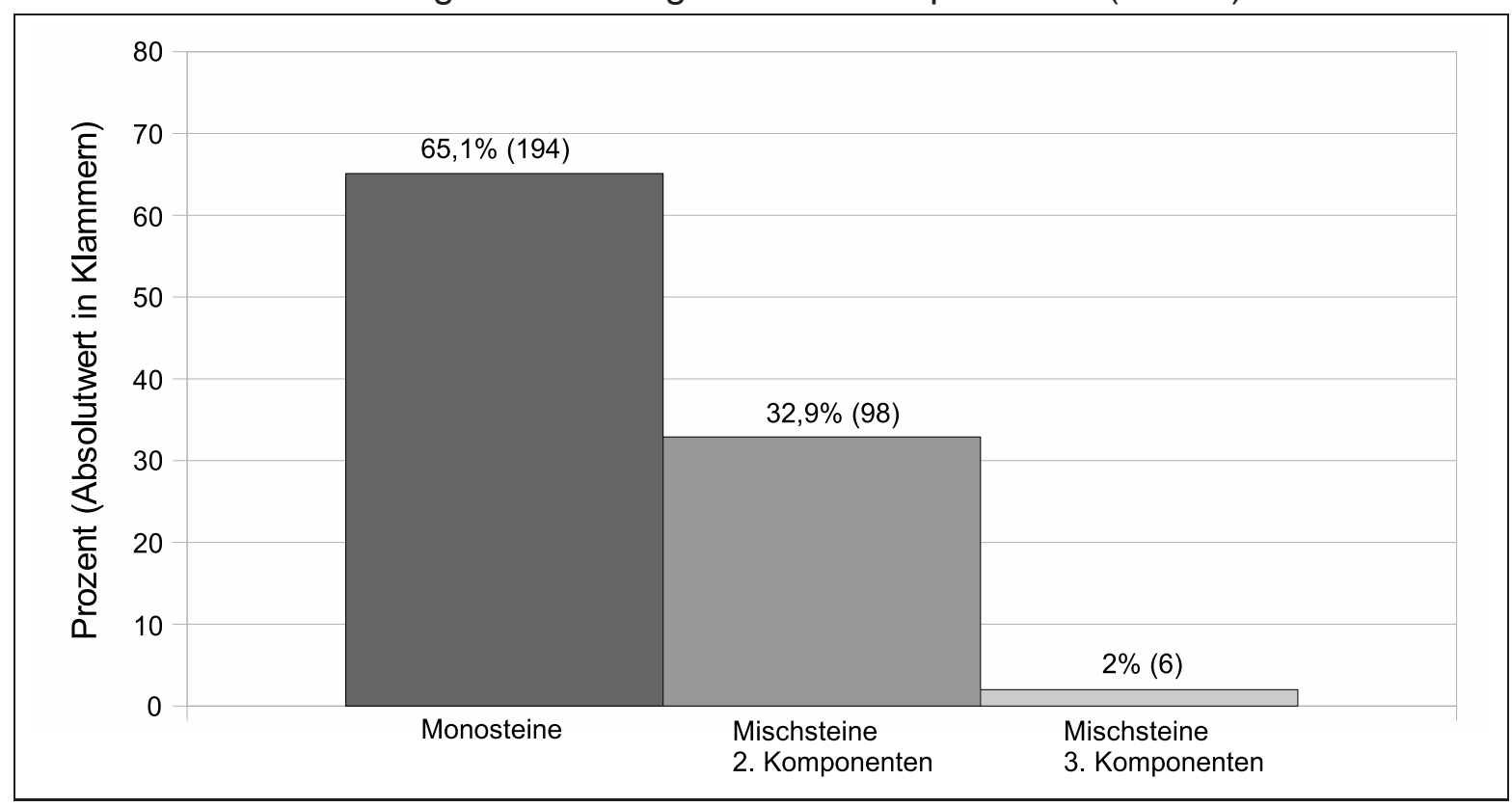

Abbildung 9: Zusammensetzung der Monosteine ( $\mathrm{n}=194)$

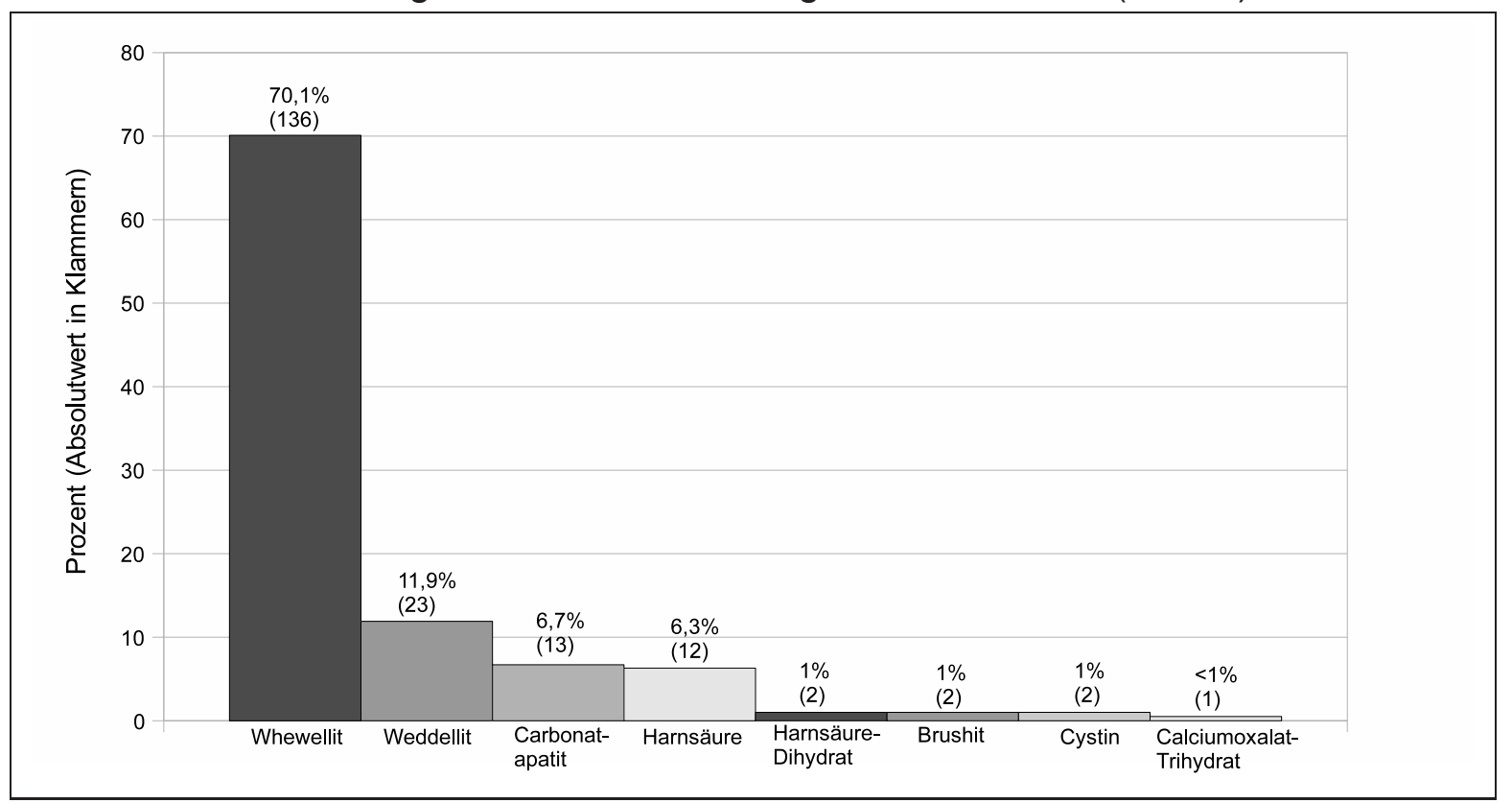

einer Erhöhung des Urin-pH-Werts führt. Patienten mit solchen Steinen haben ein erhöhtes Risiko für eine Urosepsis, da sich Harnwegsinfekt und Steinbildung gegenseitig begünstigen. Eine Metaphylaxe, komplette Steinfreiheit und die Beseitigung des Harnwegsinfekts mit entsprechender Antibiose sind therapeutische Ziele. 
Abbildung 10: Zusammensetzung der Mischsteine (104 Steine / n=214 Komponenten)

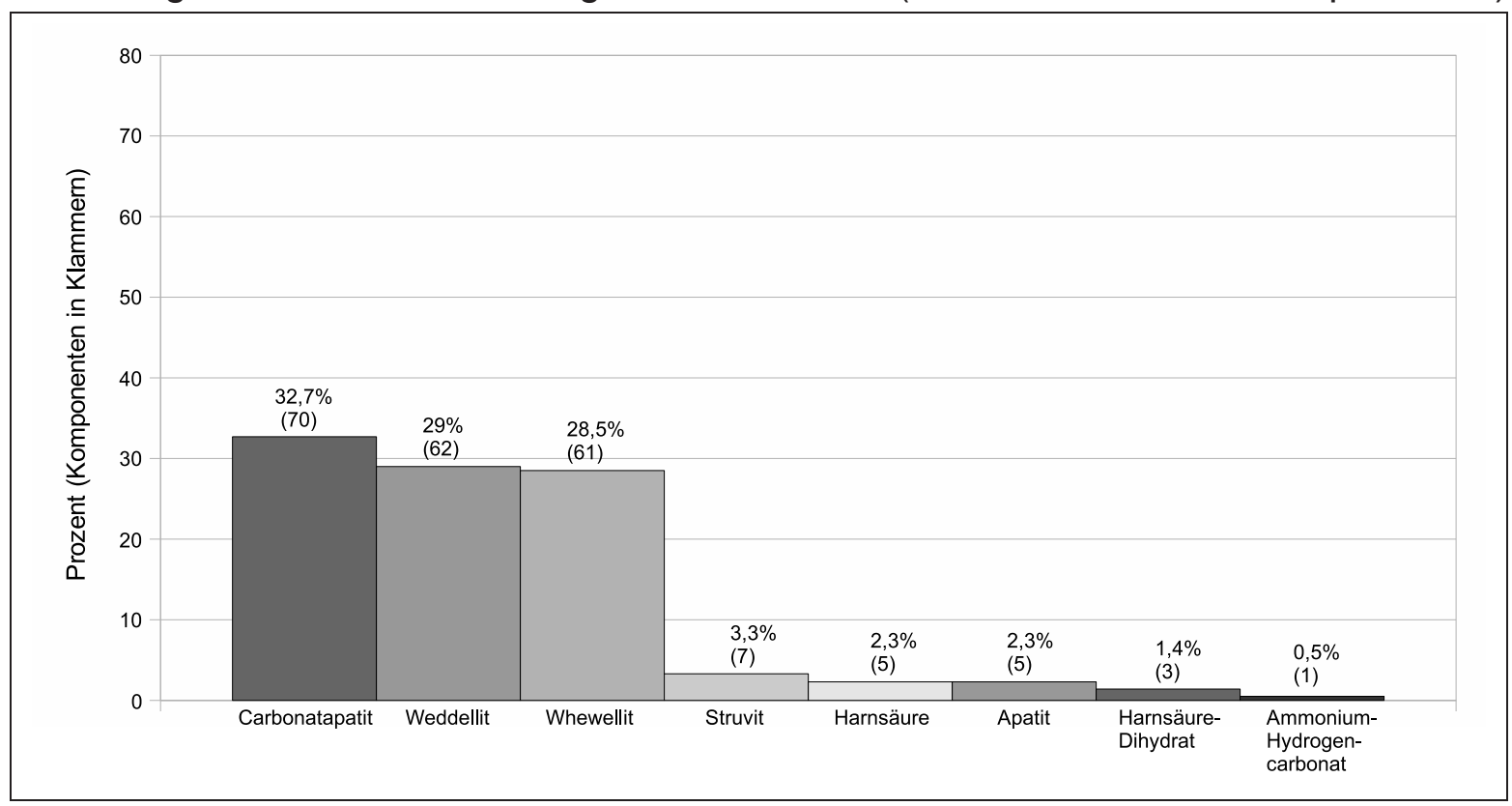

Zu den Oxalatsteinen gehören Whewellit (Calciumoxalat-Monohydrat) und Weddellit (Calciumoxalat-Dihydrat). Die Metaphylaxe dieser Harnsteine ist komplex und abhängig vom Ausscheidungsprofil des Urins.

Harnsäuresteine bilden sich in saurem, mit Harnsäure übersättigtem Urin. Die orale und lokale Chemolitholyse stellen effektive Behandlungsmöglichkeiten dar.

Brushitsteine bestehen aus Calciumhydrogenphosphat-Dihydrat und zeigen eine hohe Rezidivneigung. Ein Hyperparathyreoidismus und eine renal tubuläre Azidose stellen auszuschließende Ursachen dar.

Cystinsteine beruhen auf einem angeborenen (genetischen) Transportdefekt des $\mathrm{Ne}$ phrons für bestimmte Aminosäuren und bilden sich in Abhängigkeit vom pH-Wert des Urins. Durch Harnalkalisierung (orale Litholyse) $\mathrm{pH}>7,5$ verbessert sich die Löslichkeit des Cystins im Urin.

\subsection{Behandlung}

Alle ausgewerteten Daten stammen aus dem Fachbereich Urologie des Universitätsklinikums Göttingen und wurden zwischen dem 01.01.1998 und dem 31.12.2008 erhoben. 
Das Ergebnis der Steinbehandlung ist in Hinblick auf die erreichte Steinfreiheitsrate und aufgetretene Komplikationen dargestellt worden. Besonders mögliche Einflüsse auf den Erfolg oder Misserfolg einer Behandlung sind mit in die Auswertung eingeflossen.

\subsubsection{Steinfreiheit}

Insgesamt wurden 594 Steinbehandlungen im Ureter und Nierenbeckenkelchsystem durchgeführt, von denen 416 (70\%) nach Ende der Operation erfolgreich im Sinne einer kompletten Steinfreiheit waren. 178 (30\%) Behandlungen waren nicht erfolgreich. In die Gruppe der nicht erfolgreichen Behandlungen fallen insgesamt 33 Therapien, bei denen die verbleibenden Restkonkremente spontan abgangsfähig erschienen.

Von 553 primären URS mit dem Ziel der Steinsanierung waren 391 (70,7\%) erfolgreich. 162 (29,3\%) URS waren primär nicht erfolgreich, wobei in 29 Fällen das Steinmaterial spontan abgangsfähig erschien. In 41 Fällen wurde innerhalb einiger Tage eine Re-URS zur Steinsanierung durchgeführt, die bei 25 Patienten (61\%) zur Steinfreiheit führte. 16 Patienten (39\%) waren auch nach Re-URS noch nicht steinfrei, bei 4 dieser Patienten erschien das zurückbleibende Steinmaterial spontan abgangsfähig.

Bei 46 Behandlungen konnte trotz des dringenden Verdachts auf einen Harnleiterstein kein Stein gefunden werden. Eine andere Ursache für die Klinik bzw. konkrementverdächtige Verschattungen im Röntgenbild konnten ebenfalls nicht gefunden werden, weswegen ein unbemerkter Spontanabgang angenommen wurde.

Steinfreiheit im Ureter (ohne Hohlsystem) Bei 402 Behandlungen wurden ausschließlich Steine im Ureter behandelt. Die hier erreichte Steinfreiheitsrate betrug $81,6 \%$. Bei 192 Ureterorenoskopien, in denen mindestens ein behandelter Stein im Nierenbeckenkelchsystem lag, betrug die Steinfreiheitsrate dagegen 45,8\%.

Steinfreiheit und Steinlokalisation Die Lokalisation des Steins wurde in Hinsicht auf die resultierende Steinfreiheitsrate untersucht. Es wurden ausschließlich Patienten mit einem einzigen betroffenen Harntraktabschnitt eingeschlossen. Bei 97 Behandlungen lagen Harnsteine in unterschiedlichen Höhen vor.

Steinstraßen wurden 29 mal nachgewiesen. Die Steinlast erstreckte sich in 9 Fällen 
auf einen, in 16 Fällen auf zwei und in 4 Fällen auf 3 Ureterabschnitte. Die erreichte Steinfreiheit betrug insgesamt 34,5\%. Bei Steinstraßen in einem Ureterabschnitt $55,6 \%$, bei den über zwei Abschnitte $25 \%$ und bei den Steinstraßen über drei Ureterabschnitte ebenfalls $25 \%$.

Bei 497 Behandlungen wurde in einem einzigen Harnleiterabschnitt oder im Bereich des Hohlsystems therapiert, die angeführten Steinfreiheitsraten beziehen sich auf diese Gruppe. Im distalen Ureter wurden die meisten und erfolgreichsten Behandlungen durchgeführt, bei 219 Steinen ergab sich hier eine Steinfreiheitsrate von 90\%. Abbildung 11 zeigt die Ergebnisse der Steinbehandlung für alle Abschnitte des Harntrakts.

Abbildung 11: Steinfreiheit und Steinlokalisation $(n=497)$

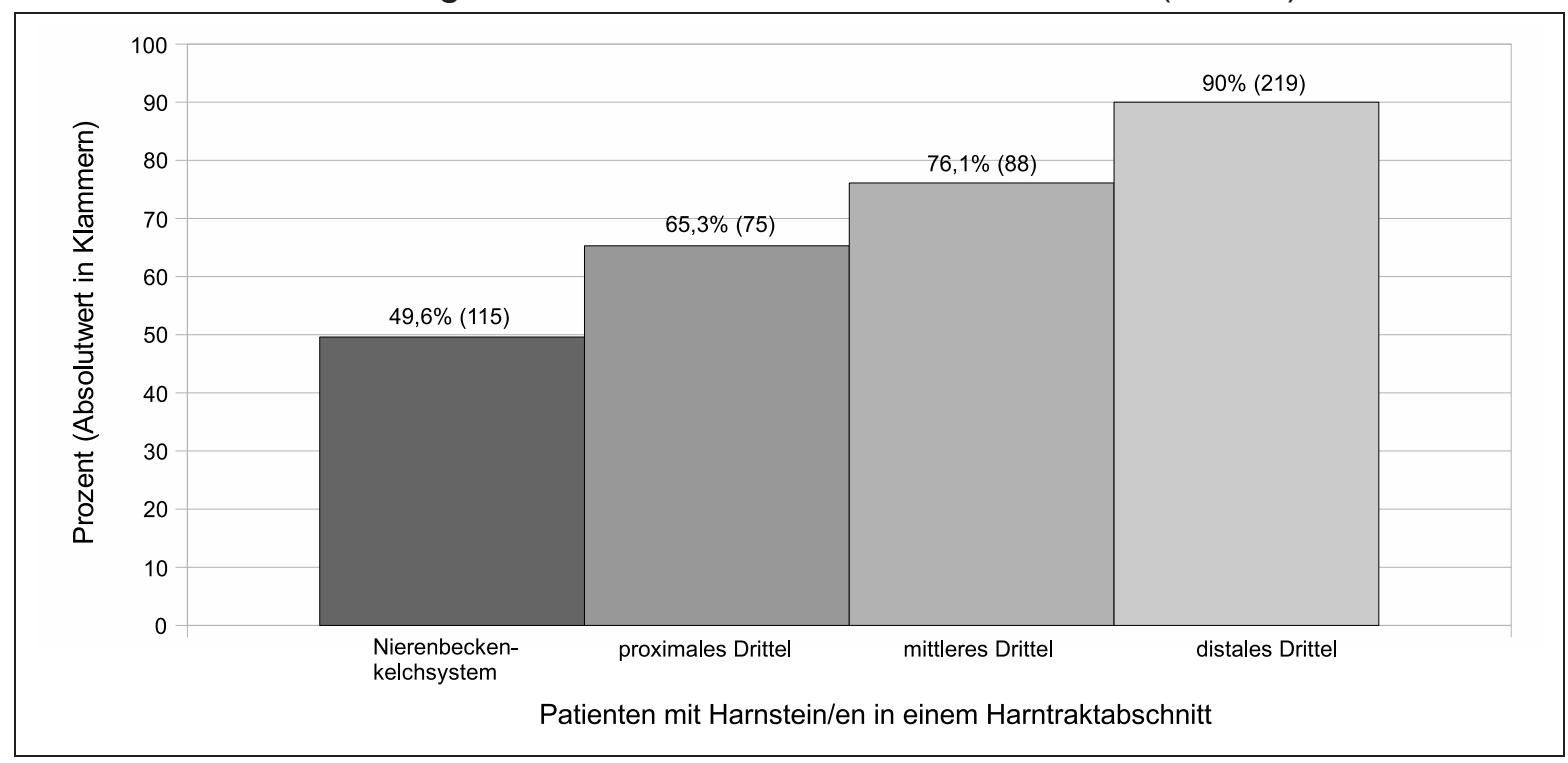

Auf der rechten Seite wurden bei Steinen in einem Harntraktabschnitt 247 Behandlungen durchgeführt mit einer Erfolgsrate von 76,1\%, auf der linken Seite wurden 250 Steine mit einer Erfolgsrate von $72,8 \%$ behandelt.

Patienten mit Steinen in unterschiedlichen Höhenabschnitten hatten bei 97 Behandlungen eine Steinfreiheitsrate von $47,1 \%$.

Steinfreiheit und Steingröße Harnsteine mit einer maximalen Kantenlänge $\leq 5 \mathrm{~mm}$ wurden am häufigsten und am erfolgreichsten behandelt (s. Abbildung 12). 26 Patienten, bei denen keine Steingröße bestimmt werden konnte, hatten eine Steinfreiheitsrate von $65,4 \%$. 
Abbildung 12: Steinfreiheit und Steingröße $(n=568)$

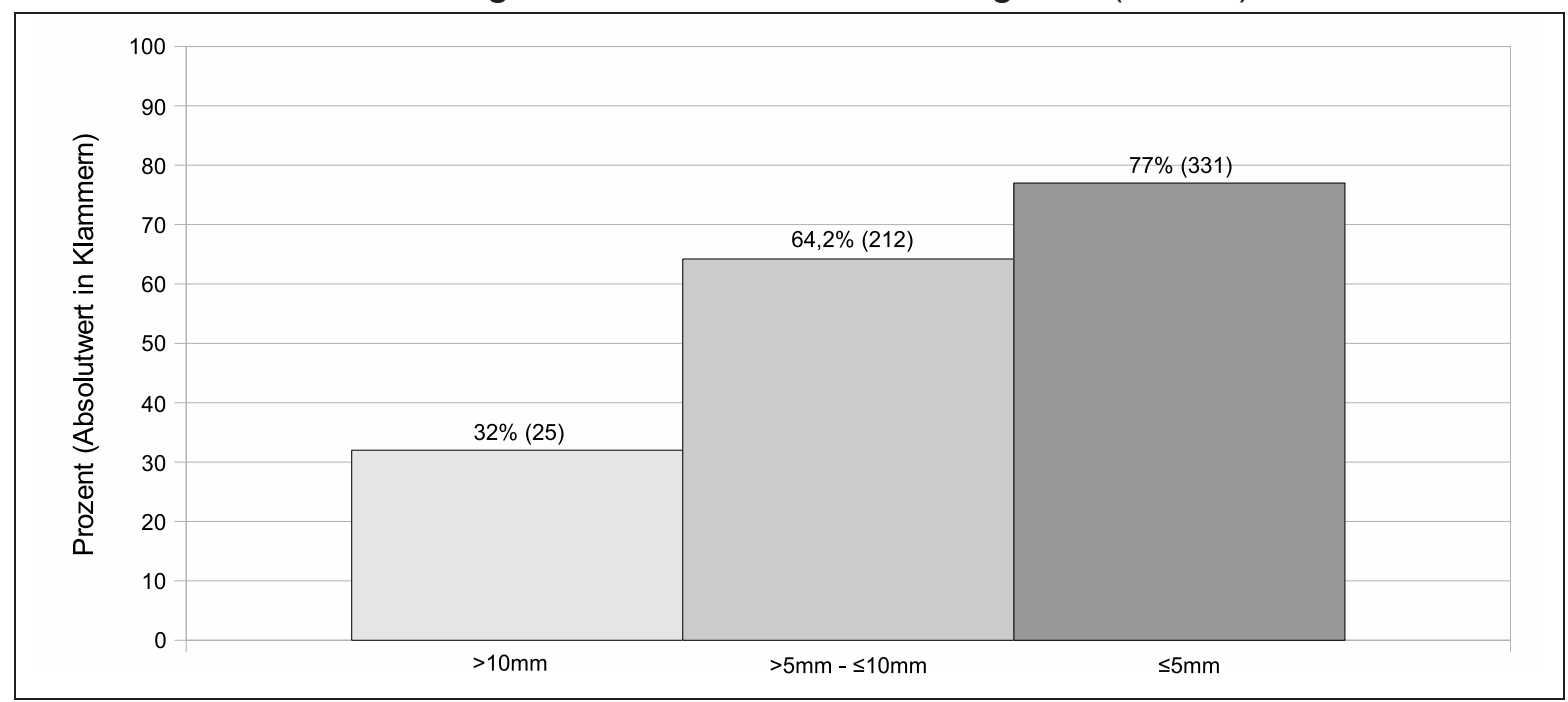

Steinfreiheit und Patientenalter Bei allen 594 Behandlungen konnte das Alter des Patienten zum Zeitpunkt der Untersuchung bestimmt werden. Zur genaueren Beschreibung der Behandlungsergebnisse wurden die Patienten in Tabelle 7 nach Lebensdekaden geordnet. Die Steinfreiheitsraten der einzelnen Gruppen wurden gegenübergestellt.

Tabelle 7: Steinfreiheit und Patientenalter

\begin{tabular}{lcr}
\hline Alter in Jahren & Patienten $(\mathrm{n}=594)$ & Steinfreiheit \\
\hline $0-9$ & 5 & $80,0 \%$ \\
$10-19$ & 12 & $66,7 \%$ \\
$20-29$ & 47 & $76,6 \%$ \\
$30-39$ & 92 & $75,0 \%$ \\
$40-49$ & 137 & $74,5 \%$ \\
$50-59$ & 113 & $67,3 \%$ \\
$60-69$ & 123 & $64,2 \%$ \\
$70-79$ & 62 & $62,9 \%$ \\
$80-89$ & 3 & $100,0 \%$ \\
\hline
\end{tabular}

Zur weiteren Auswertung wurde das Patientenkollektiv in der Nähe des mittleren Patientenalters von 49,8 Jahren in eine Gruppe $<50$ Jahre und eine Gruppe $\geq 50$ Jahre aufgeteilt. 293 Patienten unter 50 Jahren wurden zu 74,7\% steinfrei, während 301 Patienten $\geq 50$ Jahre zu $65,4 \%$ steinfrei wurden. 
Steinfreiheit und Body-Mass-Index Der BMI war präoperativ bei $569(95,8 \%)$ von 594 Behandlungen bestimmbar. Bei Mehrfachbehandlungen eines Patienten ist der BMI zu jeder einzelnen Behandlung ausgewertet worden. Es wurden nur erwachsene Patienten berücksichtigt. 10 Patienten unter 18 Jahren wurden zur besseren Vergleichbarkeit der Daten nicht mit in Tabelle 8 aufgenommen.

Tabelle 8: Steinfreiheit und Body-Mass-Index

\begin{tabular}{lcrlrr}
\hline \multicolumn{2}{l}{ Männer $\geq 18$ Jahre $(n=392)$} & \multicolumn{3}{l}{ Frauen $\geq 18$ Jahre $(n=167)$} \\
\hline BMI & Behandl. & Steinfreiheit & BMI & Behandl. & Steinfreiheit \\
\hline$<18,5$ & 4 & $75,0 \%$ & $<18,5$ & 6 & $50,0 \%$ \\
$18,5-24,9$ & 117 & $72,6 \%$ & $18,5-24,9$ & 54 & $77,8 \%$ \\
$25-30$ & 171 & $64,9 \%$ & $25-30$ & 53 & $71,7 \%$ \\
$>30$ & 100 & $69,0 \%$ & $>30$ & 54 & $72,2 \%$ \\
\hline
\end{tabular}

Steinfreiheit und Ureterorenoskope Erfolgreiche Untersuchungen wurden sowohl mit den beiden semirigiden als auch mit den flexiblen Ureterorenoskopen durchgeführt. Weiterhin kamen alle denkbaren Kombinationen der Geräte in unterschiedlicher Häufigkeit und mit abweichendem Erfolg vor.

Abbildung 13 illustriert die Steinfreiheitsrate und die Einsatzhäufigkeit anhand der fünf am häufigsten eingesetzten Geräte und deren Kombinationen. Es wurden 593 der 594 durchgeführten Behandlungen ausgewertet, in einem Fall sind die verwendeten Geräte nicht dokumentiert.

Die Steinfreiheitsrate der Geräte wurde in Abbildung 14 in Beziehung zur Lokalisation der Harnsteine gesetzt. Es wurden nur Untersuchungen eingeschlossen, bei denen der Steinnachweis in einem einzelnen Abschnitt des Harnleiters oder dem Nierenbeckenkelchsystem erfolgt war. Patienten mit Steinen in mehreren Abschnitten wurden nicht berücksichtigt. Abbildung 14 zeigt die fünf am häufigsten verwendeten Geräte und deren Kombinationen. Insgesamt wurden 477 Behandlungen ausgewertet.

Steinfreiheit und Instrumentarium Bei 572 von 594 Behandlungen (96,3\%) wurden Instrumente in der Operationsdokumentation erwähnt. Insgesamt wurden 781 Instrumente eingesetzt, bei 389 Behandlungen (68\%) wurde intraoperativ nur ein Instrument und bei 183 Behandlungen (32\%) wurden mehrere Instrumente verwendet. 
Abbildung 13: Steinfreiheit und Ureterorenoskope (Die 5 häufigsten Geräte und Gerätekombinationen)

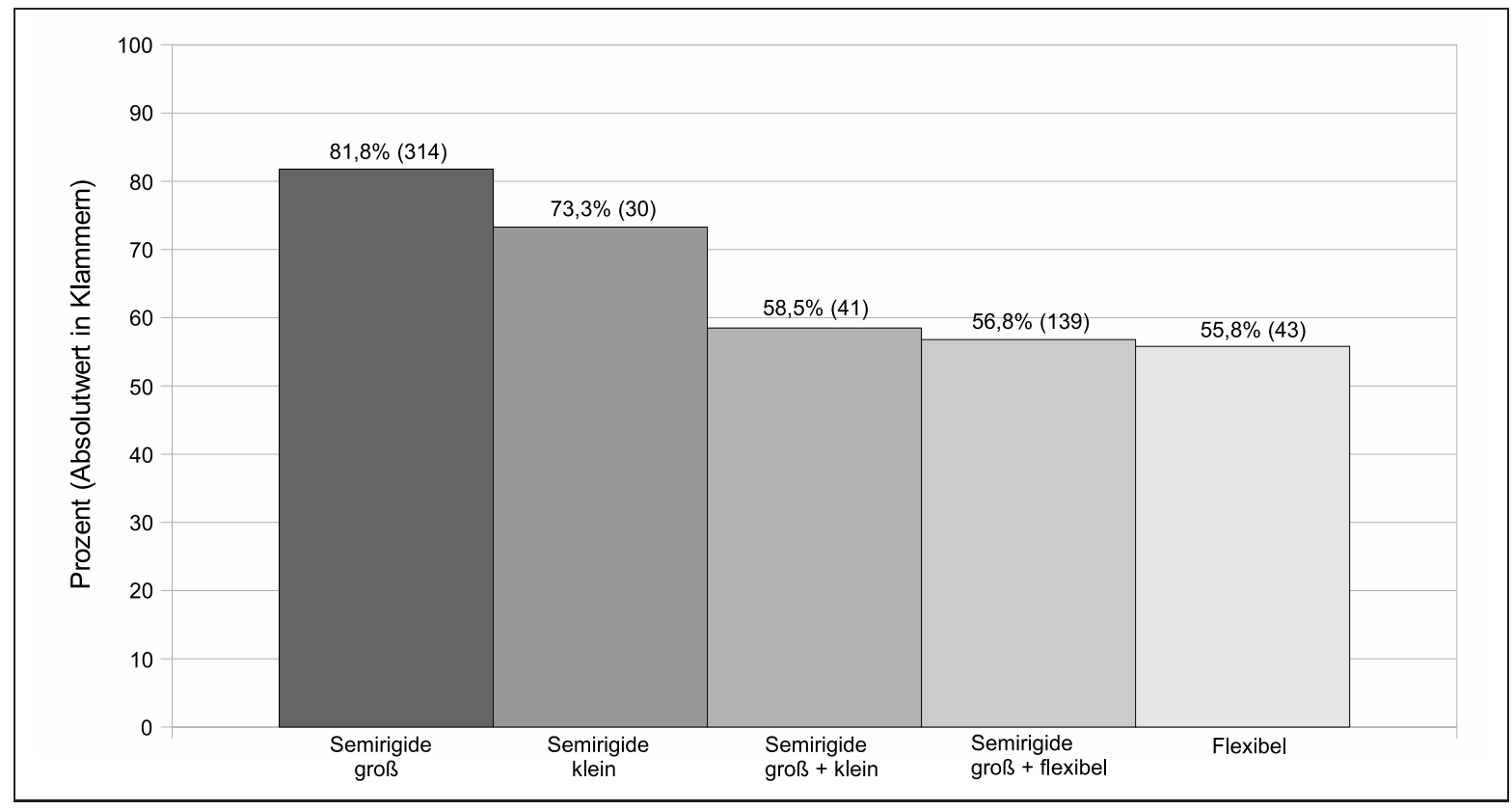

Abbildung 14: Steinfreiheit und Ureterorenoskope in Bezug zur Steinlokalisation

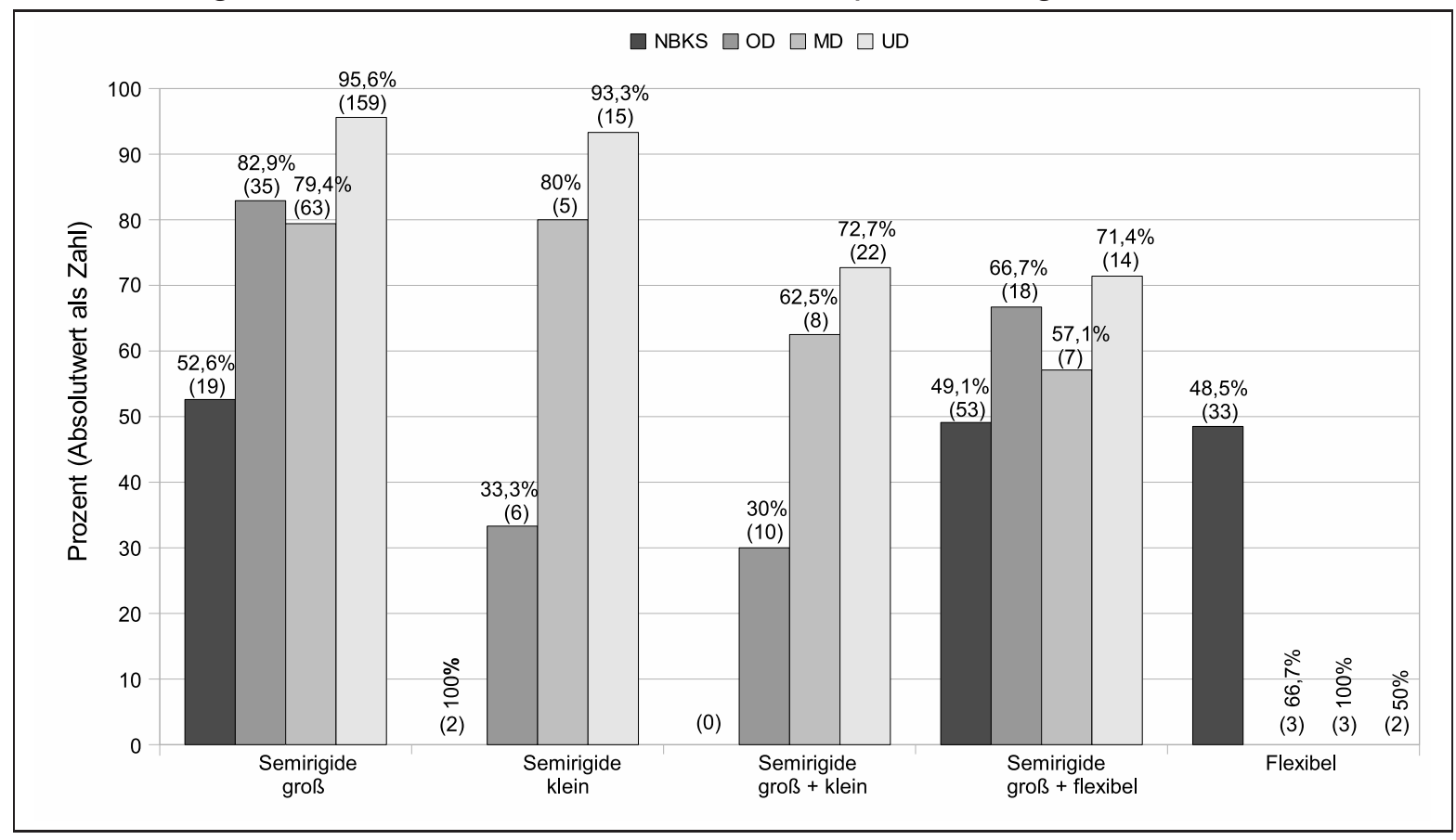


Tabelle 9 zeigt die erreichte Steinfreiheitsrate bei Behandlungen mit bestimmten Instrumenten und Instrumentkombinationen.

Tabelle 9: Steinfreiheit und Instrumentarium

\begin{tabular}{lcr}
\hline Instrument(e) & Behandlungen $(\mathrm{n}=572)$ & Steinfreiheit \\
\hline Körbchen & 353 & $82,7 \%$ \\
Körbchen + Laser & 132 & $56,1 \%$ \\
Fasszange + Körbchen + Laser & 24 & $54,2 \%$ \\
Fasszange & 17 & $70,6 \%$ \\
Fasszange + Körbchen & 17 & $64,7 \%$ \\
Laser & 15 & $13,3 \%$ \\
Fasszange + Laser & 5 & $60,0 \%$ \\
Sonotrode & 3 & $33,3 \%$ \\
Sonotrode + Körbchen & 2 & $50,0 \%$ \\
Sonotrode + Fasszange + Körbchen & 2 & $50,0 \%$ \\
Sonotrode + Fasszange & 1 & $100,0 \%$ \\
Zeiss'sche Schlinge & 1 & $0,0 \%$ \\
\hline
\end{tabular}

Es zeigte sich, dass das Dormiakörbchen und die Kombination aus Dormiakörbchen und Laser in $485(84,8 \%)$ der 572 Behandlungen Verwendung fand. In der weiteren Auswertung werden diese beiden Instrumente genauer untersucht.

Das Körbchen wird vor allem im distalen Ureter mit sehr guten Erfolgsraten bis 97,2\% eingesetzt. Die Kombination von Körbchen und Laser wird im Vergleich dazu häufiger im proximalen Teil des Ureters verwendet (s. Abbildung 15).

Kleine und mittlere Steine $(\leq 10 \mathrm{~mm}$ ) werden häufig mit dem Körbchen komplett entfernt. Die Behandlung größerer Steine setzt eine Lithotripsie voraus, die mit dem Ho:YAG-Laser durchgeführt werden kann. Tabelle 16 zeigt die Erfolgsrate der Instrumente in Abhängigkeit der behandelten Harnsteingröße. 
Abbildung 15: Steinfreiheit und Instrumente in Bezug zur Steinlokalisation

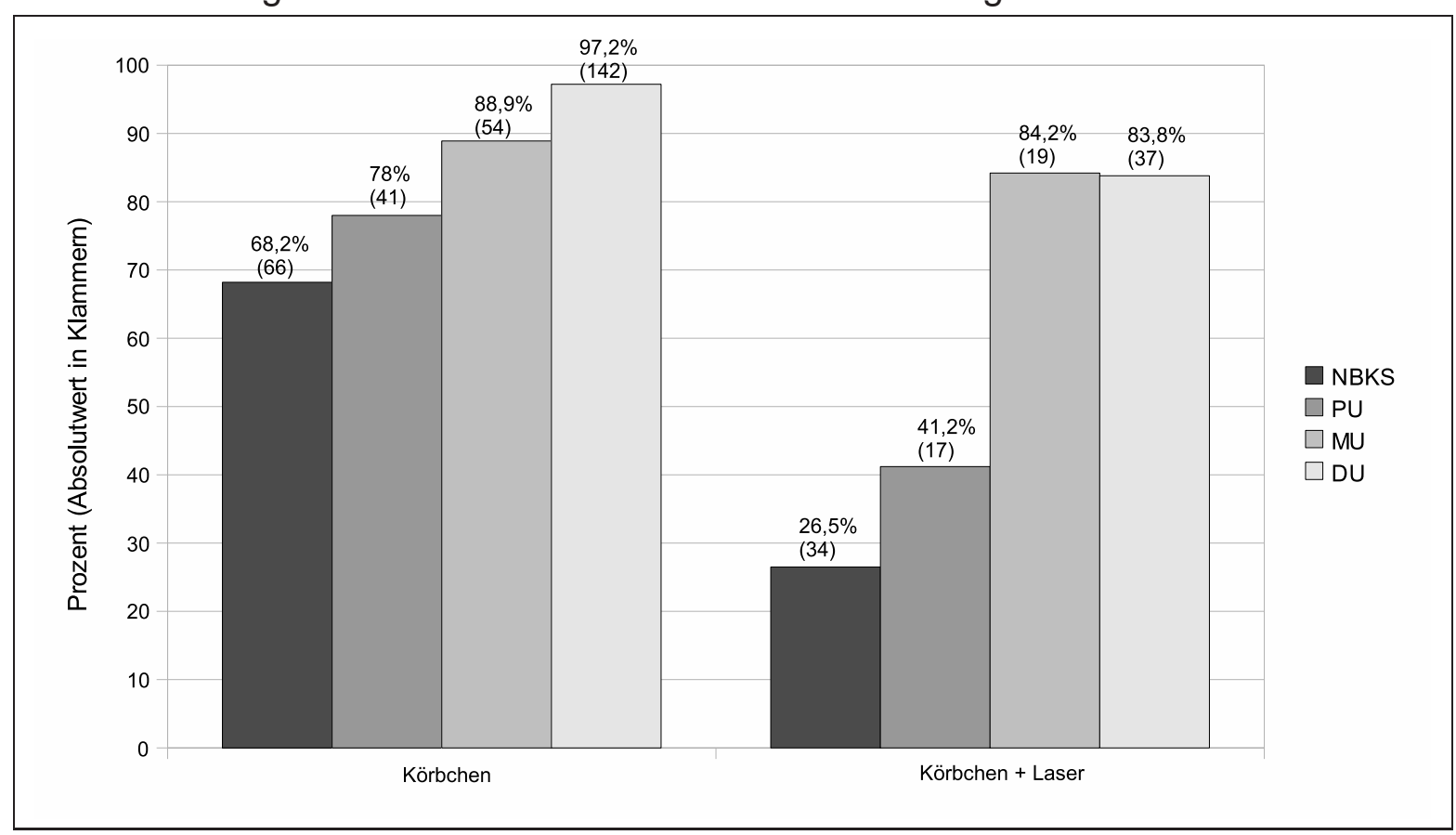

Abbildung 16: Steinfreiheit und Instrumente in Bezug zur Steingröße

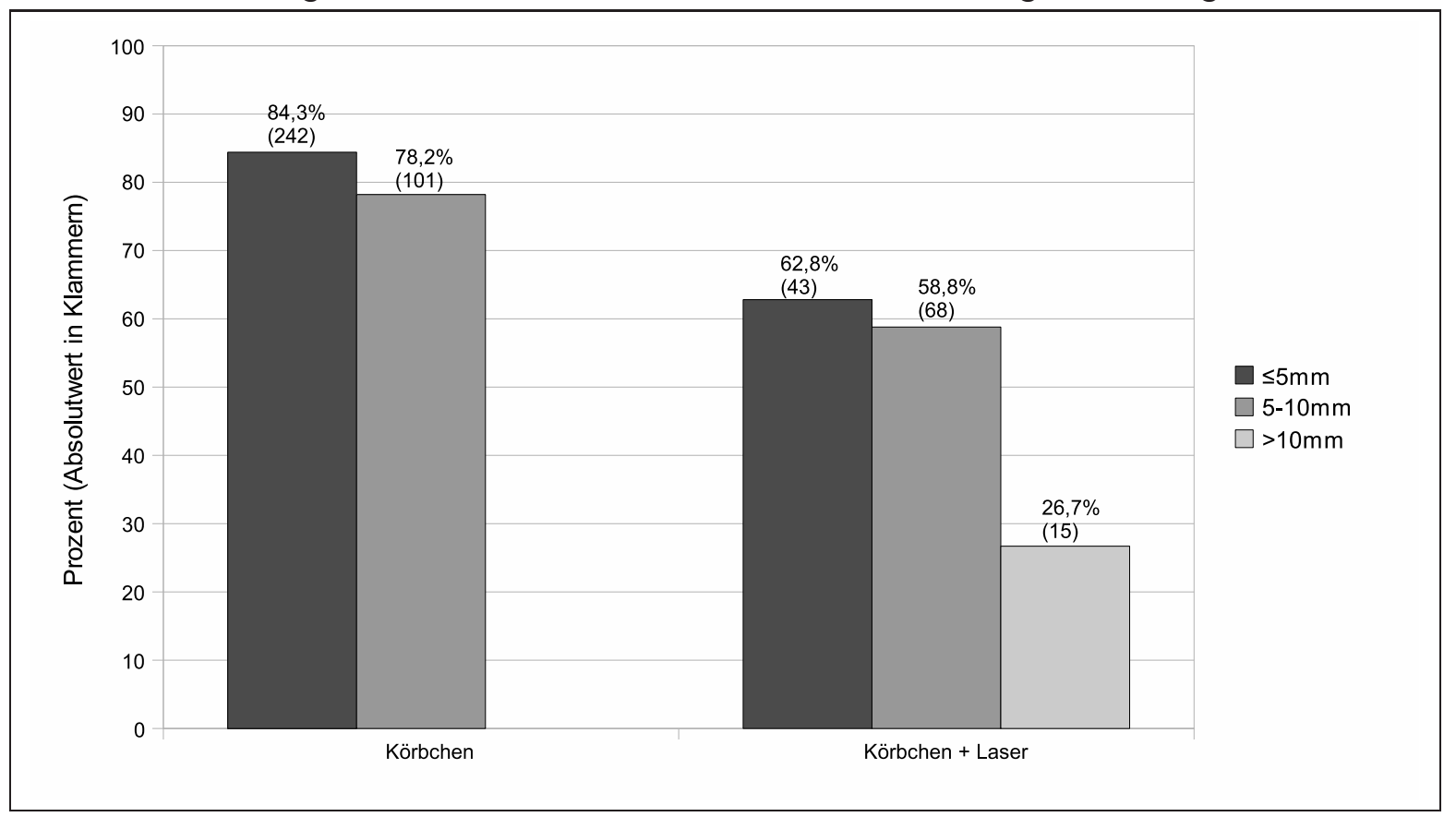




\subsubsection{Komplikationen}

Bei 59 von 594 Behandlungen von Harnsteinen im Ureter oder Hohlsystem kam es zu Komplikationen. Die Gesamtkomplikationsrate betrug 9,9\%. Bei 54 Patienten handelte es sich um eine einzelne Komplikation, 5 Patienten hatten zwei Komplikationen. Tabelle 10 zeigt die Art und Häufigkeit der ausgewerteten Komplikationen.

Zur Erfassung intra- und perioperativer Komplikationen standen der OP-Bericht sowie der Anästhesiebogen zur Verfügung. Postoperative Komplikationen konnten aus den Patientenkurven und den Entlassungsbriefen entnommen werden.

Tabelle 10: aufgetretene Komplikationen insgesamt ( $n=59$ Behandlungen)

\begin{tabular}{lrlr}
\hline eine Komplikation & Anzahl & zwei Komplikationen & Anzahl \\
\hline Harnleiter-Perforation & 16 & Harnleiter-Perforation + Fieber & 3 \\
Nierenbecken-Perforation & 9 & Nierenbecken-Perforation + Fieber & 1 \\
Harnleiterabriss & 2 & & \\
Fieber & 15 & & \\
Urosepsis & 1 & & 1 \\
Harnabflussstörung & 9 & Harnabflussstörung + Fieber & \\
Blutung & 2 & & \\
(mit Transfusionsbedarf) & & & \\
\hline
\end{tabular}

Es wurden insgesamt 19 Harnleiterperforationen (3,2\%) beobachtet. Intraoperativ wurden diese durch Kontrastmittelapplikation dargestellt und dokumentiert. Es kam zu 4 Perforationen im proximalen Drittel des Harnleiters, 9 im mittleren Drittel und 4 im distalen Drittel. Bei 2 Perforationen waren auf dem Röntgenbild die Austrittsstellen nicht klar zu erkennen.

Bei 2 Behandlungen (0,3\%) kam es zu einem Ureterabriss, welcher offen operativ versorgt werden musste. In beiden Fällen war der Ureterverlauf im distalen Drittel unterbrochen.

Bei 20 Patienten $(3,4 \%)$ kam es postoperativ zu einer Fieberentwicklung mit Temperaturen über 38,5 Grad Celsius (rektal gemessen).

Major- und Minorkomplikationen Majorkomplikationen sind in dieser Studie die Harnleiter- und Nierenbeckenperforation, der Harnleiterabriss, die Urosepsis sowie die transfusionsbedürftige Blutung. Es handelt sich um Komplikationen, die eine unverzügliche und oft invasive Behandlung benötigen. Zu den Minorkomplikationen wurden 
das postoperative Fieber und die Harnabflussstörung gezählt. Minorkomplikationen in dieser Arbeit zeichnen sich dadurch aus, dass eine notwendige Therapie schnell, aber nicht unverzüglich eingeleitet werden muss und die Komplikationen oft konservativ behandelt werden können.

Früh- und Spätkomplikationen Zu den Frühkomplikationen zählt in dieser Arbeit die Harnleiter- und Nierenbeckenperforation sowie der Harnleiterabriss. Es handelt sich dabei um Komplikationen, die intra- oder perioperativ entstanden sind und in aller Regel noch vor Abschluss der Operation erkannt werden. Zu den Spätkomplikationen, welche erst im Verlauf der postoperativen stationären Behandlung auftreten, zählen das postoperative Fieber, die Urosepsis, die Harnabflussstörung und die transfusionsbedürftige Blutung.

Abbildung 17 zeigt die Gesamtkomplikationsrate aller untersuchten Ureterorenoskopien dieser Studie und teilt diese zur weiteren Veranschaulichung in die unten beschriebenen Gruppen ein.

Abbildung 17: Gesamtkomplikationsrate der URS

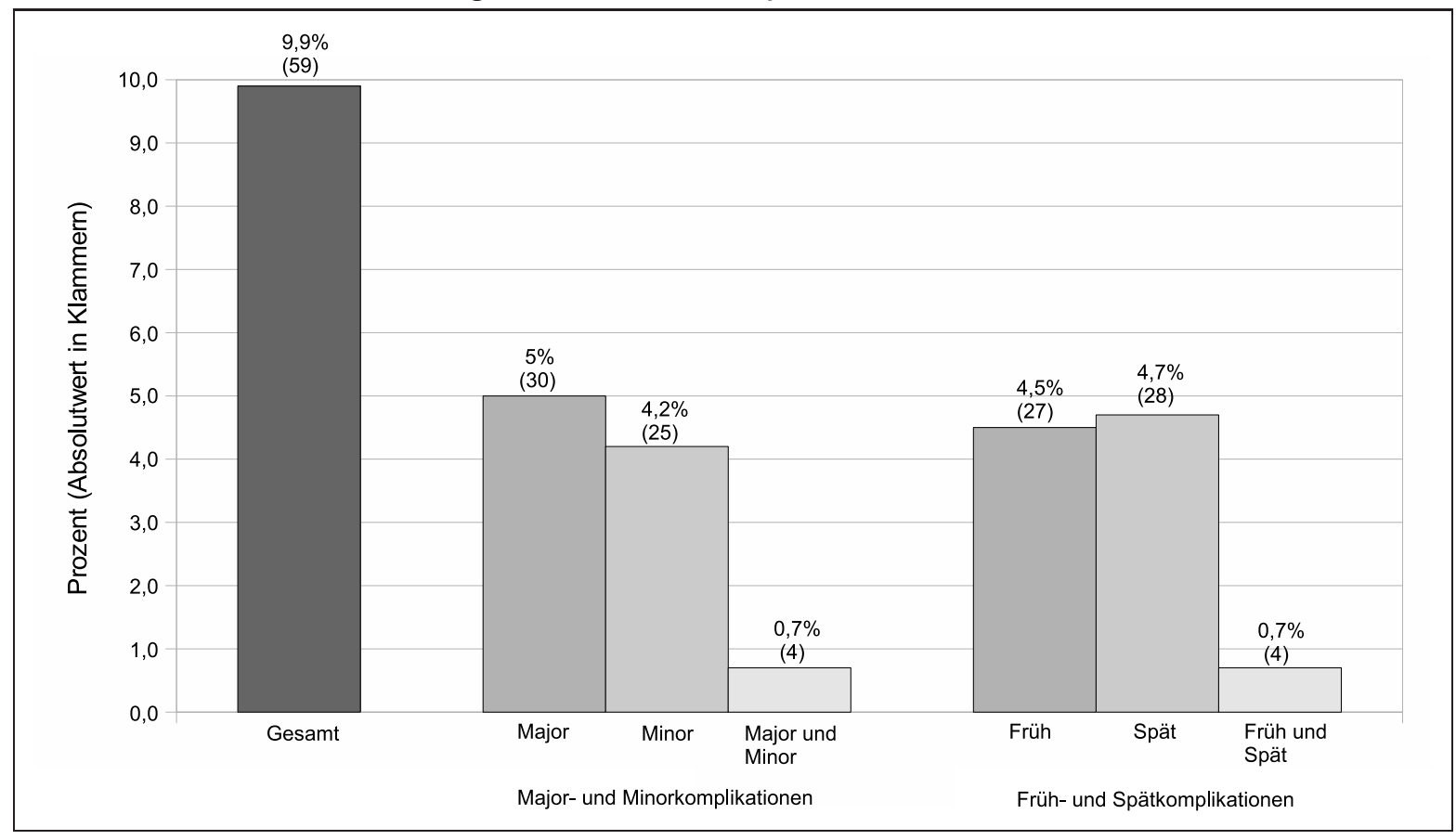

Komplikationen im Ureter (ohne Hohlsystem) Bei 402 Behandlungen $(67,7 \%)$ von Steinen im Ureter (ohne Hohlsystem) kam es in 39 Fällen zu Komplikationen, die Kom- 
plikationsrate betrug 9,7\%. 36 Patienten zeigten eine Komplikation, 3 Patienten zwei Komplikationen. Es wurden 22 (5,5\%) Major, 15 (3,7\%) Minor sowie 2 (0,5\%) Majorund Minorkomplikationen beobachtet. Bei den Uretersteinen wurden 19 (4,7\%) Früh-, $18(4,5 \%)$ Spät- und $2(0,5 \%)$ Früh- und Spätkomplikationen dokumentiert.

Komplikationen und Steingröße Abbildung 18 beschreibt das Auftreten von Komplikationen in Abhängigkeit von der behandelten Steingröße. Die Harnsteine wurden anhand der maximalen Kantenlänge gruppiert, was bei 568 von 594 Behandlungen möglich war. Bei 26 Behandlungen war die Steingröße nicht bestimmbar. Es trat in dieser Gruppe eine Komplikation auf.

Abbildung 18: Komplikationen und Steingröße $(n=568)$

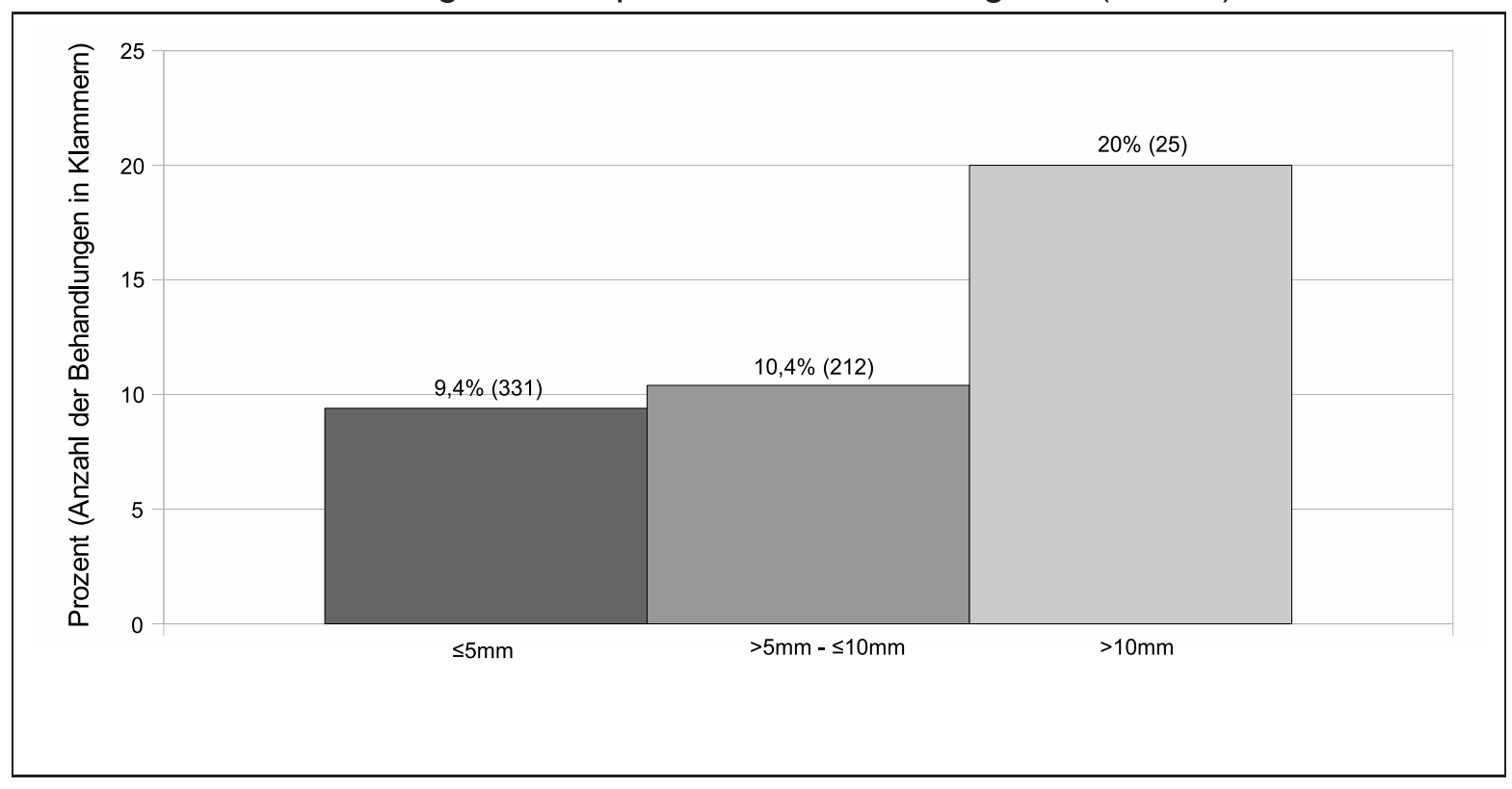

Wird die Größe der behandelten Harnsteine mit den aufgetretenen Komplikationen verglichen, zeigt sich eine Häufung von Perforationen bei Steinen $>10 \mathrm{~mm}$. Es traten 3 von 19 Harnleiterperforationen (15,8\%) und 2 von 10 Nierenbeckenperforationen (20\%) bei Steinen $>10 \mathrm{~mm}$ auf, wobei diese nur einen Anteil von 4,4\% an der Gesamtsteinzahl einnahmen. Andere Komplikationen zeigten kein gehäuftes Auftreten bei der Behandlung von Harnsteinen $>10 \mathrm{~mm}$.

Komplikationen und Steinlokalisation Bei $7(11,9 \%)$ von 59 Behandlungen mit Komplikationen war mehr als eine Steinlokalisation beschrieben worden. Um die Kom- 
plikationen einem Harnleiterabschnitt bzw. dem Nierenbeckenkelchsystem zuordnen zu können, wurden Steinträger mit Steinen in mehreren Abschnitten als getrennte Gruppe behandelt.

Abbildung 19: Komplikationen und Steinlokalisation $(n=594)$

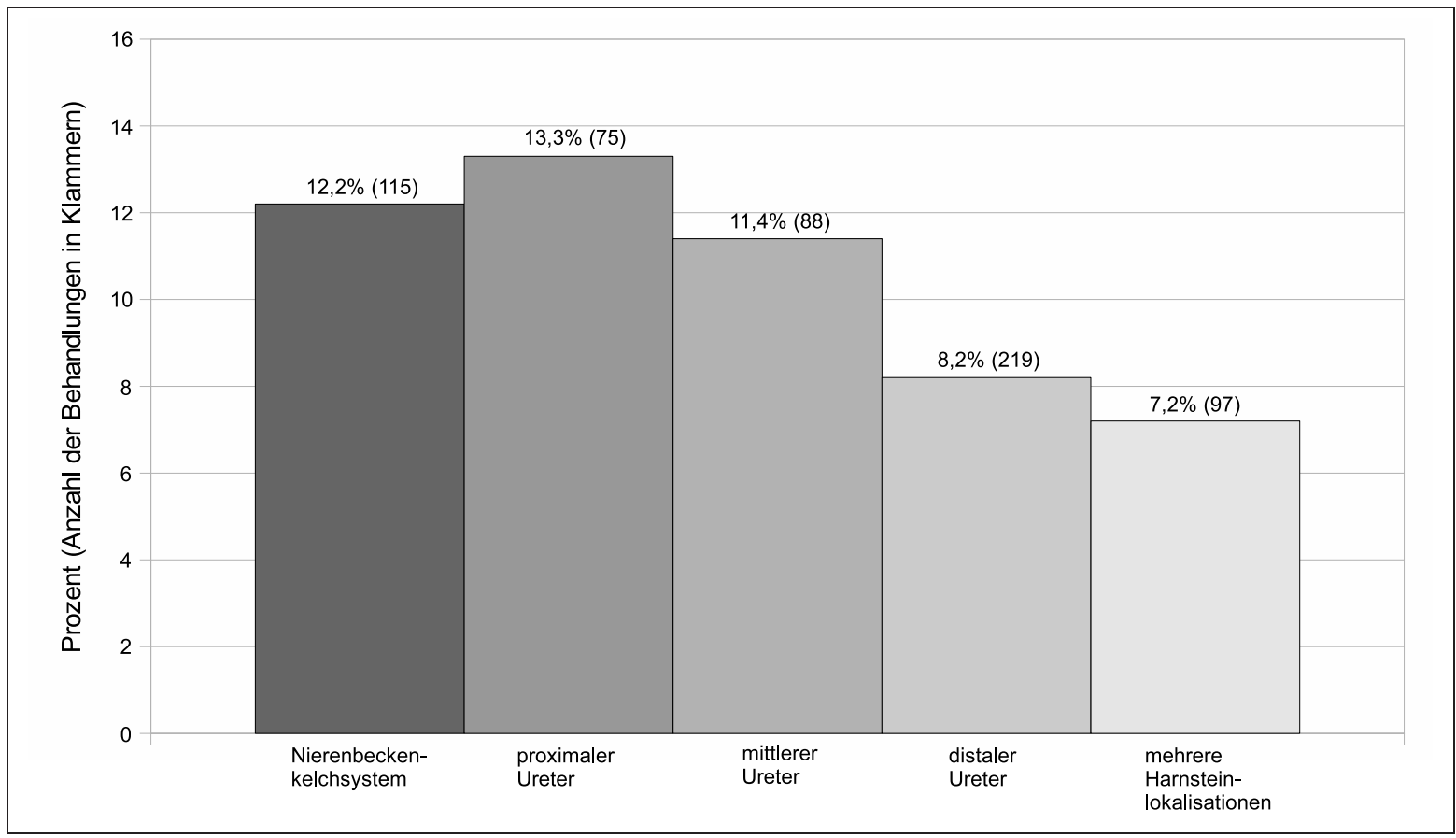

Wie in Abbildung 19 zu sehen, sank die Komplikationsrate von 13,3\% im proximalen Ureter auf 8,2\% im distalen Ureter.

Eine erhöhte Komplikationsrate im proximalen Harntrakt ist besonders bei 2 Arten von Komplikationen zu finden gewesen. Obwohl im proximalen und mittleren Ureter nur $32,8 \%$ der Steine behandelt wurden, traten 13 von 19 der Harnleiterperforationen $(68,4 \%)$ hier auf. Weitere 2 Harnleiterperforationen (10,5\%) traten bei Patienten mit Steinen in mehreren Lokalisationen des proximalen Harntrakts auf.

Bei insgesamt 20 Fällen von postoperativem Fieber wurde ein Harnstein 7 mal im Hohlsystem (35\%) und 3 mal im proximalen Ureter (15\%) behandelt. Es entfallen damit 50\% der Fieberkomplikationen auf diesen Bereich, obwohl nur 38,2\% der Steine dort lokalisiert waren. 3 weitere Fälle (15\%) von postoperativen Fieber gehen auf Patienten mit mehreren behandelten Harnsteinlokalisationen zurück, davon mindestens ein Harnstein im Hohlsystem. 
Komplikationen und Patientenalter Dem Alter der Patienten in Lebensdekaden wurden in Tabelle 11 die aufgetretenen Komplikationen zugeordnet.

Tabelle 11: Komplikationen und Patientenalter

\begin{tabular}{lcrr}
\hline $\begin{array}{l}\text { Patientenalter } \\
\text { (in Jahren) }\end{array}$ & $\begin{array}{c}\text { Patienten } \\
(\mathrm{n}=594)\end{array}$ & $\begin{array}{r}\text { Anzahl Kompl. } \\
(\mathrm{n}=59)(9,9 \%)\end{array}$ & Komplikationsrate \\
\hline $0-9$ & 5 & $1(0,2 \%)$ & $20,0 \%$ \\
$10-19$ & 12 & $0(0,0 \%)$ & $0,0 \%$ \\
$20-29$ & 47 & $6(1,0 \%)$ & $12,8 \%$ \\
$30-39$ & 92 & $6(1,0 \%)$ & $6,5 \%$ \\
$40-49$ & 137 & $9(1,5 \%)$ & $6,6 \%$ \\
$50-59$ & 113 & $10(1,7 \%)$ & $8,8 \%$ \\
$60-69$ & 123 & $16(2,6 \%)$ & $13,0 \%$ \\
$70-79$ & 62 & $10(1,7 \%)$ & $16,1 \%$ \\
$80-89$ & 3 & $1(0,2 \%)$ & $33,3 \%$ \\
\hline
\end{tabular}

Das mittlere Alter des hier untersuchten Patientenkollektivs beträgt 49,8 Jahre. Die Gruppe der Patienten <50 Jahre umfasst 293 Personen (49,3\%) und die Gruppe der Patienten $\geq 50$ Jahre 301 Personen (50,7\%). Drei der beobachteten Komplikationen zeigten eine deutliche Altersverteilung. Harnleiterperforationen wurden bei 5 Patienten $<50$ Jahren $(26,3 \%)$ und 14 Patienten $>50$ Jahren $(73,7 \%)$ beobachtet. Nierenbeckenperforationen bei 4 Patienten der jüngeren Gruppe (44,4\%) und 6 Patienten der älteren Gruppe (66,6\%). Postoperatives Fieber trat bei 8 Patienten <50 Jahren (40\%) und bei $12 \geq 50$ Jahren auf $(60 \%)$.

Abbildung 20 zeigt, bei wieviel Prozent der Behandlungen der einzelnen Gruppe Komplikationen auftraten.

Komplikationen und Body-Mass-Index Die Komplikationen wurden anhand der WHO-Gliederung der Gewichtsklassen für Erwachsene eingeteilt (World Health Organization 2004). Es zeigte sich, dass von 10 minderjährigen Patienten 7 unter der BMI Grenze von 18,5 kg/m² lagen (70\%). Bei den Erwachsenen waren es 10 Patienten bei 559 Behandlungen (1,8\%). Um die Vergleichbarkeit der Werte zu verbessern, wurden nur Behandlungen von erwachsenen Patienten ( $\geq 18$ Jahre) ausgewertet.

Abbildung 21 zeigt Komplikationsraten der URS in Abhängigkeit vom Body-Mass-Index der Patienten. In die Abbildung wurden die Patientendaten von 559 Behandlungen integriert. Bei 25 Patienten konnte der BMI Wert nicht bestimmt werden. Diese Gruppe 
Abbildung 20: Komplikationen und Patientenalter

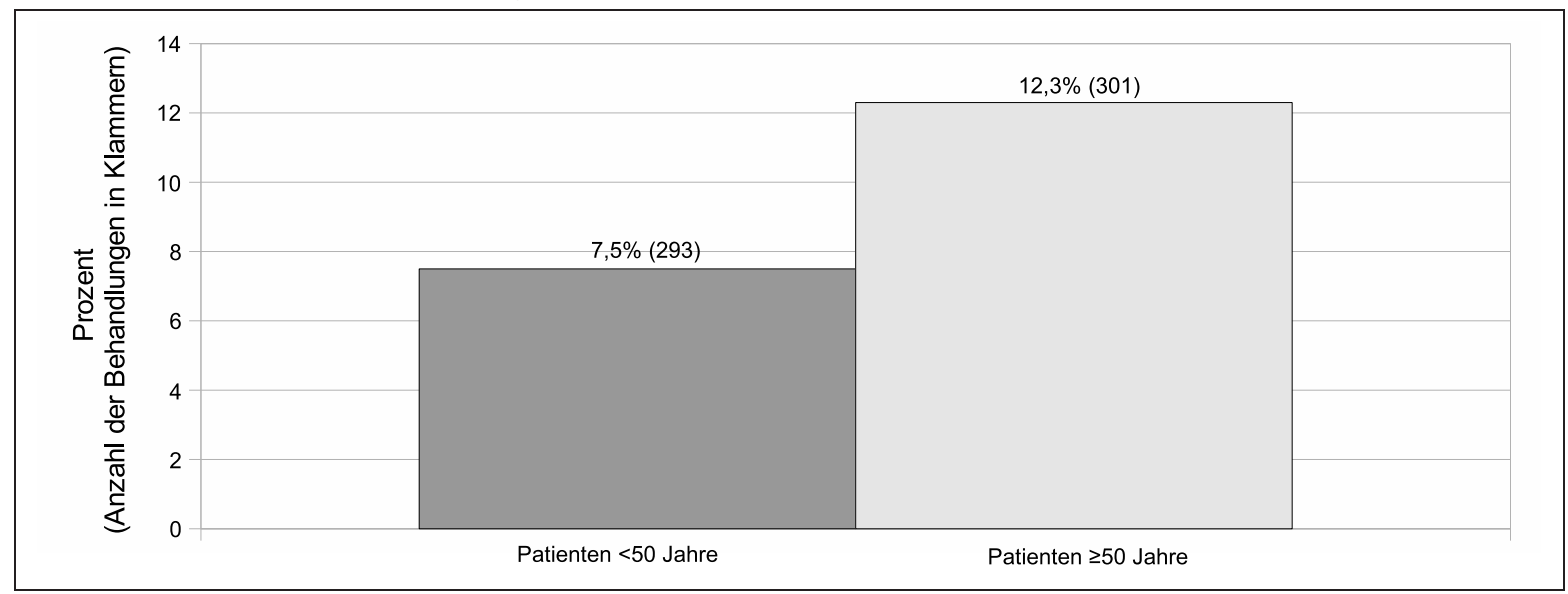

zeigte 3 Komplikationen. 10 weitere Patienten, die nicht in Abbildung 21 aufgeführt sind waren minderjährig (<18 Jahre). Bei diesen Patienten wurde 1 Komplikation beobachtet.

Abbildung 21: Komplikationen und Body-Mass-Index

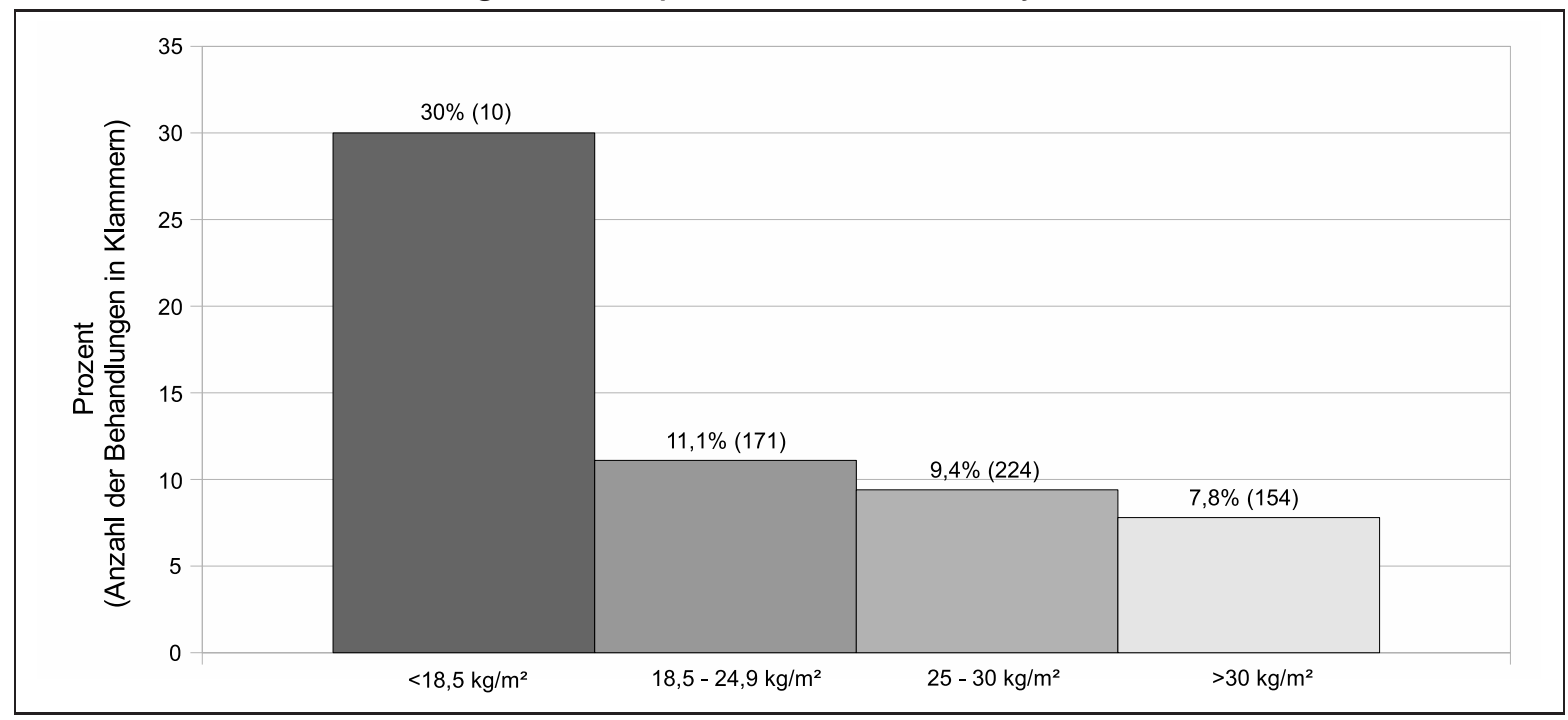

$60 \%$ der Nierenbeckenperforationen ereigneten sich bei „übergewichtigen“ Patienten mit einem BMI von $25-30 \mathrm{~kg} / \mathrm{m}^{2}$, obwohl diese nur einen Anteil von $40,1 \%$ am erwachsenen Patientenkollektiv ausmachen. Auffällig war, das adipöse Patienten mit einem $\mathrm{BMI}>30 \mathrm{~kg} / \mathrm{m}^{2}$ tendenziell weniger Komplikationen entwickelten. Bei 27,5\% adipösen Patienten wurden nur 15\% (3/20) der Fälle postoperativen Fiebers und 20\% (2/10) der Fälle einer Harnabflussstörung beobachtet. Die einzige Urosepsis entfiel allerdings 
auf einen adipösen Patienten. Die restlichen Komplikationen zeigten keine Häufungen bei bestimmten BMI Gruppen.

Komplikationen und Ureterorenoskope Die fünf am häufigsten verwendeten Geräte und Gerätekombinationen und die spezifische Komplikationsrate bei ihrer Verwendung werden in Tabelle 12 aufgeführt.

Tabelle 12: Komplikationen und Ureterorenoskope

\begin{tabular}{lcrr}
\hline $\begin{array}{l}\text { Geräte und } \\
\text { Kombinationen }\end{array}$ & $\begin{array}{c}\text { Behandlungen } \\
(\mathrm{n}=594)\end{array}$ & $\begin{array}{r}\text { Anzahl Kompl. } \\
(\mathrm{n}=59)(9,9 \%)\end{array}$ & Komplikationsrate \\
\hline semirigide groß & 314 & $24(4,0 \%)$ & $7,6 \%$ \\
semirigide groß + flexibel & 139 & $15(2,5 \%)$ & $10,8 \%$ \\
flexibel & 43 & $4(0,7 \%)$ & $9,3 \%$ \\
semirigide groß + klein & 41 & $7(1,2 \%)$ & $17,1 \%$ \\
semirigide klein & 30 & $6(1,0 \%)$ & $20,0 \%$ \\
andere & 26 & $3(0,5 \%)$ & $11,5 \%$ \\
unbekannt & 1 & $0(0,0 \%)$ & $0,0 \%$ \\
\hline
\end{tabular}

Bei Verwendung des semirigiden Ureterorenoskops mit dem kleineren Kaliber (7,8 Ch Schaftdicke) zeigte sich ein Anstieg der Komplikationen. Bei den Harnleiterperforationen entfielen 6 von 19 Perforationen $(31,6 \%)$ auf die Kombination des großen mit dem kleinen semirigiden Ureterorenoskop, obwohl diese Kombination nur in 6,9\% der Operationen Verwendung fand.

Komplikationen und Instrumentarium Es wurden die beiden mit 84,8\% am häufigsten eingesetzten Instrumentmöglichkeiten Körbchen und Körbchen plus Laser untersucht (s. Tabelle 13).

Tabelle 13: Komplikationen und Instrumentarium

\begin{tabular}{lcrr}
\hline $\begin{array}{l}\text { Instrumente } \\
\text { und Kombination }\end{array}$ & $\begin{array}{c}\text { Behandlungen } \\
(\mathrm{n}=594)\end{array}$ & $\begin{array}{r}\text { Anzahl Kompl. } \\
(\mathrm{n}=59)(9,9 \%)\end{array}$ & Komplikationsrate \\
\hline Körbchen & 353 & $29(4,9 \%)$ & $8,2 \%$ \\
Körbchen + Laser & 132 & $14(2,3 \%)$ & $10,6 \%$ \\
andere Instrumente & 87 & $11(1,9 \%)$ & $12,6 \%$ \\
nicht angegeben & 22 & $5(0,8 \%)$ & $22,7 \%$ \\
\hline
\end{tabular}


Bei den meisten beobachteten Komplikationen zeigte sich, dass die Verwendung der Instrumentenkombination Körbchen + Laser (23,1\% der auswertbaren Operationen) eine gering höhere Komplikationsrate nach sich zog, als die Verwendung des Körbchens als Monoinstrument (61,7\% der Operationen). Eine Ausnahme stellten die Harnabflussstörungen dar, von denen 80\% (8 von 10) nach Körbchen und 10\% (1 von 10) nach Körbchen plus Laser Behandlung auftraten, 10\% (1 von 10) entfielen auf eine andere Instrumentenkombination. 


\section{Diskussion}

Patientendemographie Geavlete et al. (2006) publizierten eine Studie, in der die Alters- und Geschlechtsmerkmale der mit dem Ureterorenoskop behandelten Patienten beschrieben wurden. Die Behandlung im Ureter erfolgte aus unterschiedlichen diagnostischen und therapeutischen Gründen. Fuganti et al. (2008) sowie El-Nahas et al. (2009) berichteten über die Patientendemographie bei rein therapeutischen ureterorenoskopischen Operationen mit dem Ziel der Steinsanierung im Harnleiter. Tabelle 14 zeigt die Ergebnisse dieser Publikationen in Bezug auf die Patientendemographie und stellt die Ergebnisse der Göttinger Studie gegenüber.

Tabelle 14: Die Patientendemographie in der Literatur

\begin{tabular}{lcccr}
\hline Studie & Patientenzahl & Männer & Frauen & Alter \\
\hline Diese Studie & 509 & $68,6 \%$ & $31,4 \%$ & $7-84$ Jahre \\
\hline El-Nahas et al. (2009) & 841 & $67,4 \%$ & $32,6 \%$ & $2-81$ Jahre \\
Fuganti et al. (2008) & 1235 & $64,9 \%$ & $35,1 \%$ & $5-76$ Jahre \\
Geavlete et al. (2006) & 2436 & $59,1 \%$ & $40,1 \%$ & $5-87$ Jahre \\
\hline
\end{tabular}

Das mittlere Alter der Patienten in Göttingen betrug 49,8 Jahre. Es zeigte sich, dass etwa ein Drittel des Patientenkollektivs Frauen (31,4\%) und etwa zwei Drittel $(68,6 \%)$ Männer waren. Die in Göttingen behandelten Patienten schienen sich in Bezug auf Alter, Geschlechts- und Altersspanne nicht wesentlich von anderen ureterorenoskopisch behandelten Steinpatienten zu unterscheiden.

Mehrfachbehandlungen Hesse et al. (2003) berichteten über eine geschätzte Rezidivrate bei Harnsteinen von $42 \%$ in einer bundesdeutschen epidemiologischen Studie. El-Nahas et al. (2009) behandelten 8\% vom 841 Patienten mehrfach an im Ureter lokalisierten Harnsteinen.

In Göttingen wurden 63 Patienten (12,4\%) mehrfach und 446 Patienten einmal an einem Harnstein behandelt. Preminger et al. (2007) beschrieben, dass abhängig von ihrer Größe bis zu 68\% der Harnsteine spontan abgehen. Für nicht spontan abgegangene Harnsteine stehen verschiedene Behandlungsmethoden zur Verfügung, so dass nicht alle Patienten mit Rezidivsteinen eine weitere Ureterorenoskopie erhalten haben. Ebenfalls sollte berücksichtigt werden, dass die Patienten den Behandlungsort frei wählen konnten und in dieser Studie ausschließlich Daten der Universitätsklinik 
Göttingen ausgewertet wurden. Zusammenfassend kann die in dieser Studie ermittelte Rezidivrate nicht die tatsächliche Anzahl von Patienten mit einem erneuten Steinereignis widerspiegeln. Epidemiologische Studien wie die von Hesse et al. (2003) können die oben beschriebenen Probleme vermeiden und liefern sicherlich Ergebnisse, die näher an den tatsächlichen Werten liegen.

Vorerkrankungen Vor allem in den Industrienationen steigt die Prävalenz von Erkrankungen, welche potentiell die Entstehung von Harnsteinen fördern könnten.

Hesse et al. (2003) berichteten über eine in Deutschland von 1979 bis 2000 angestiegene Inzidenz von ersten Steinereignissen bei Patienten zwischen 25 und 50 Jahren. In der Studie werden veränderte Lebens- und Ernährungsgewohnheiten als mögliche begünstigende Faktoren dieser Entwicklung genannt. Die Inzidenz der Urolithiasis in der deutschen Gesamtbevölkerung stieg ebenfalls deutlich von 0,54\% im Jahr 1979 auf $1,47 \%$ im Jahr 2000. Auch hier werden veränderte Lebens- und Ernährungsgewohnheiten als wichtige Gründe diskutiert.

Sakhaee (2008) publizierte eine Studie über die Verbindung der Nephrolithiasis mit dem metabolischen Syndrom. Die Studie berichtete über eine positive Korrelation der beiden Erkrankungen und schlug vor, das Vorliegen einer Nephrolithiasis als Symptom einer systemischen Erkrankung anzusehen. Ursächlich seien vermutlich verschiedene Komponenten des metabolischen Syndroms (Übergewicht, Diabetes mellitus, Hypertonus und Hyperlipoproteinämie) an der Entstehung der Nierensteinerkrankung beteiligt. Die Studie beschreibt weiter, dass ein im Vergleich zu Gesunden niedrigerer Urin pH-Wert bei Diabetikern in einem erhöhten Risiko für Harnsäuresteine resultiert.

Calvert und Burgess (2005) veröffentlichten das Ergebnis einer Literatursuche zu dem Thema Urolithiasis bei Übergewicht. In mehreren epidemiologischen Studien fanden sie den Zusammenhang von Übergewicht und Harnsteinleiden bestätigt. Zahlreiche pathophysiologische Mechanismen scheinen der veränderten Urinzusammensetzung übergewichtiger Menschen zugrunde zu liegen und damit auch eine höhere Wahrscheinlichkeit für bestimmte Harnsteintypen zu verursachen.

Adeghate et al. (2006) berichteten zum Thema Diabetes mellitus von einer weltweiten Prävalenz von etwa 6\%, die weiter ansteigt. Die Prävalenz dieser endokrinen Erkrankung wird für Europa zwischen $0,7 \%$ und $11,6 \%$ angegeben. Gellner und Domschke (2008) veröffentlichten eine Studie, in der von einer geschätzten weltweiten Prävalenz des Übergewichts (BMI von >25kg/m²) von 1,1 Milliarden Menschen und einer Präva- 
lenz der Adipositas (BMI von $>30 \mathrm{~kg} / \mathrm{m}^{2}$ ) von 312 Millionen Personen berichtet wurde. Die Studie beschrieb zudem einen Anstieg der weltweiten Prävalenz um den Faktor 2 bis 3 innerhalb der letzten 20 Jahre. In Deutschland beträgt die Prävalenz der Adipositas (BMI von $>30 \mathrm{~kg} / \mathrm{m}^{2}$ ) bei erwachsenen Personen 22,9\% und bei Kindern und Jugendlichen 6,3\%. Prugger et al. (2006) publizierten, dass Deutschland im internationalen Vergleich mit einer Prävalenz der Hypertonie von 55\% im Oberfeld liegt. 26\% der Hypertoniker erhalten in Deutschland eine Therapie im Vergleich zu 58\% in den USA. Smith DG (2007) berichtet in einem Review zur Prävalenz der Hypercholesterinämie in den USA von etwa 30\% der Bevölkerung mit Werten $>200$ mg/dl und etwa $10 \%$ über 240 mg/dl mit dringend indiziertem Behandlungsbedarf.

Im Göttingen Patientenkollektiv waren 26,3\% der Patienten „adipös“ mit einem BMI $>30 \mathrm{~kg} / \mathrm{m}^{2}$. Dieser Wert liegt etwas über dem deutschen Mittelwert von 22,9\% bei erwachsenen Personen. Da der Body-Mass-Index bei den meisten Patienten dieser Arbeit $(91,9 \%)$ berechnet werden konnte, ist davon auszugehen, dass der errechnete Wert der tatsächlichen Prävalenz sehr nahe kommt. Bei 9,6\% der Patienten wurde ein Diabetes mellitus festgestellt, bei $7,3 \%$ eine Hyperlipoproteinämie und bei $24,2 \%$ ein Hypertonus. Da die Erfassung der Vorerkrankungen retrospektiv anhand der in den Patientenakten notierten Medikamente und Diagnosen erfolgte, ist nicht gesichert, dass jeder erkrankte Patient als solcher identifiziert werden konnte. Besonders die in dieser Arbeit ausgewertete Prävalenz der Hypertonie von 24,2\% weicht deutlich von der für Deutschland angenommenen Prävalenz von 55\% ab. Auch wenn bekannt ist, dass in Deutschland nur etwa $26 \%$ der Hypertoniker behandelt werden, hätte die Erkrankung als vorbeschriebene Diagnose vermutlich häufiger auftauchen müssen. Zur retrospektiven Berechnung des Body-Mass-Index waren die archivierten Daten aus den Patientenakten ausreichend. Zur genauen Prävalenzbestimmung der Hyperlipoproteinämie, der Hypertonie und des Diabetes mellitus wäre eine direkte Patientenbefragung bzw. die klinische Untersuchung optimal gewesen. Vor diesem Hintergrund sind die ermittelten Häufigkeiten dieser Erkrankungen kritisch zu sehen.

Harnsteinlokalisation Geavlete et al. (2006), El-Nahas et al. (2009) und Sözen et al. (2003) veröffentlichten Studien, in denen die Steinlokalisation bei zahlreichen Ureterorenoskopien bei Harnsteinen im Ureter (ohne Hohlsystem) beschrieben wurde. Ihre Ergebnisse im Vergleich zu den Daten dieser Studie zeigt Tabelle 15.

In dieser Studie hatten 97 Patienten Steine in mehreren Abschnitten des Harntrakts, 
Tabelle 15: Die Harnsteinlokalisationen in der Literatur

\begin{tabular}{lccrrr}
\hline Studie & Behandlungen & NBKS & PU & MU & DU \\
\hline Diese Studie (NB + Ureter) & 497 & $23,1 \%$ & $15,1 \%$ & $17,7 \%$ & $44,1 \%$ \\
Diese Studie (Ureter) & 382 & - & $19,6 \%$ & $23,0 \%$ & $57,4 \%$ \\
\hline El-Nahas et al. (2009) & 908 & - & $18,6 \%$ & $19,4 \%$ & $62,0 \%$ \\
Sözen et al. (2003) & 500 & - & $7,2 \%$ & $9,4 \%$ & $83,4 \%$ \\
Geavlete et al. (2006) & 2041 & - & $38,5 \%$ & - & $61,5 \%$ \\
\hline
\end{tabular}

so dass sie in einer eigenen Gruppe zusammengefasst wurden und nicht in die oben genannte Auswertung einflossen. Beide Gruppen zeigten jedoch die meisten Steine im Nierenbeckenkelchsystem und distalen Ureter.

Die Einteilung der Steine im Harntrakt ist in der internationalen Literatur uneinheitlich geregelt. Einige Autoren unterscheiden nur in einen proximalen und distalen Ureter, andere zusätzlich in einen mittleren Ureterabschnitt. In dieser Studie wurde neben dem oberen, mittleren und unteren Ureterabschnitt zusätzlich das Nierenbeckenkelchsystem als relevanter Ort für ein Steinereignis erfasst. Die ureterorenoskopische Steinbehandlung im NBKS war durch den Einsatz flexibler Geräte in vielen Fällen möglich, wodurch oftmals auf eine PCNL-Behandlung verzichtet werden konnte. Die Möglichkeit, Harnsteine auch im Hohlsystem mit dem Ureterorenoskop zu behandeln, führte in der vorliegenden Arbeit zur Berücksichtigung dieser proximalen Steinlokalisation. Vergleichbar mit den in Tabelle 15 gezeigten Literaturergebnissen ist in Göttingen die Mehrzahl der Harnleitersteine im distalen Ureter behandelt worden. Im proximalen und mittleren Ureter sind weitaus seltener Harnsteine lokalisiert gewesen.

Harnsteingröße Tabelle 16 zeigt die von Fuganti et al. (2008) und Sözen et al. (2003) publizierten Harnsteingrößen bei der ureterorenoskopischen Behandlung.

Tabelle 16: Die Harnsteingröße in der Literatur

\begin{tabular}{lcccc}
\hline Studie & Anzahl Steine & Mittlere Steingröße & minimal & maximal \\
\hline Diese Studie & 568 & $5,4 \mathrm{~mm}$ & $1,5 \mathrm{~mm}$ & $18 \mathrm{~mm}$ \\
\hline Fuganti et al. (2008) & 1235 & $8,0 \mathrm{~mm}$ & $3,0 \mathrm{~mm}$ & $60 \mathrm{~mm}$ \\
Sözen et al. (2003) & 500 & $8,7 \mathrm{~mm}$ & & \\
\hline
\end{tabular}

Der durchschnittliche in Göttingen behandelte Harnstein hatte eine maximale Kan- 
tenlänge von 5,4 mm. Die „S2-Leitlinien zur Diagnostik, Therapie und Metaphylaxe der Urolithiasis“ beschrieben Harnsteine von $<4 \mathrm{~mm}$ als spontan abgangsfähig, wobei Konkremente dieser Größe bereits durchschnittlich mehrere Wochen zum Abgang benötigten (Knoll et al. 2009 a). Zu beachten ist ebenfalls, dass in manchen Fällen die Steine durch frustrane auxiliäre Maßnahmen wie die ESWL zum Teil desintegriert vorlagen. Dies verringerte die in der Röntgenaufnahme messbare maximale Steingröße. Ein großer Teil $(58,3 \%)$ der in dieser Studie erfassten Harnsteine war $\leq 5 \mathrm{~mm}$ groß und konnte mit einer durchschnittlichen Steinfreiheitsrate von $77 \%$ behandelt werden. Die guten Erfolgsraten der Ureterorenoskopie in Göttingen bei kleinen und distal gelegenen Harnsteinen führten dazu, dass viele Steine $\leq 5 \mathrm{~mm}$ bevorzugt mit der URS behandelt wurden.

Harnsteinanalyse Knoll et al. (2009 a) veröffentlichten eine Übersicht zur Harnsteinzusammensetzung. Sie gaben eine Häufigkeit von 60\% für Mischsteine und $40 \%$ für monomineralische Steinen an. Schubert (2006) publizierte eine Auswertung von mehr als 110000 Harnsteinanalysen, in der sich 66\% der untersuchten Steine aus mehreren Komponenten und 34\% der Steine aus einer Komponente zusammensetzten. Whewellit konnte in $78 \%$, Weddellit in $43 \%$ und Apatit in 33\% der ausgewerteten Harnsteinanalysen nachgewiesen werden. Weitere Steinkomponenten wurden in $\leq 10 \%$ der Fälle gefunden.

In dieser Studie enthielten von 298 ausgewerteten Steinen 65,1\% eine Komponente (Monosteine) und 34,9\% zwei oder drei Komponenten (Mischsteine). Bei dem Monosteinen dominierte mit 70,1\% Calciumoxalat-Monohydrat (Whewellit), während bei den Mischsteinen Whewellit und Weddellit (Calciumoxalat-Dihydrat) ungefähr in gleicher Menge vorkamen und zusammen 57,5\% der Steinmasse ausmachten. Carbonatapatit wurde bei 6,7\% der Monosteine und bei 32,7\% der Mischsteine nachgewiesen. Es wurde von Seiten des Labors eine chemische Harnsteinkomponente als vorhanden angesehen, wenn sie mehr als 5\% der Steinmasse ausmachte. Die Häufigkeit, mit der chemische Harnsteinkomponenten in dieser Arbeit gefunden wurden, stimmte weitestgehend mit den Ergebnissen der internationalen Literatur überein. Die 3 in der Literatur am häufigsten genannten Komponenten Whewellit, Weddellit und Apatit waren auch in Göttingen am stärksten repräsentiert. Das Verhältnis der monomineralischen Steine zu den Mischsteinen stimmte allerdings nicht mit den Literaturergebnissen überein. In dieser Arbeit war der überwiegende Teil der Harnsteine $(65,1 \%)$ monomineralisch aufgebaut, während verschiedene Publikationen von $\leq 40 \%$ berichteten. Dieses Ergebnis 
sollte vor dem Hintergrund der unterschiedlich großen Zahl ausgewerteter Harnsteinanalysen betrachtet werden. Für diese Arbeit konnten 298 Analysen ausgewertet werden, während in Publikationen teils mehr als 100000 Steinanalysen einflossen. Die Ergebnisse der Literatur geben daher vermutlich ein genaueres Bild über die Verteilung der Mono- und Mischsteine wieder.

Steinfreiheit Steinfreiheit wurde in dieser Arbeit als komplettes Entfernen aller nachweisbaren Steinfragmente definiert. Die Erfolgsrate bei 594 Steinbehandlungen im Nierenbeckenkelchsystem und Ureter lag bei 70\%. Bei 402 Behandlungen von Konkrementen im Ureter (ohne Hohlsystem) wurde eine Steinfreiheitsrate von $81,6 \%$ erreicht.

Steinfreiheit und Steinlokalisation Tabelle 1 zeigt die zusammengefassten Steinfreiheitsraten vieler Studien in Abhängigkeit von der Lokalisation der Konkremente. Die Daten beruhen zum Teil auf den 2007 erschienenen Leitlinien zur Behandlung von Harnsteinen der "European Association of Urology (eau)". Darin wurde über Steinfreiheitsraten absteigend vom proximalen zum distalen Ureter von $81 \%, 86 \%$ und $94 \%$ berichtet. Preminger et al. (2007) publizierten, dass proximale Harnsteine im Vergleich zu distalen Steinen eine geringere Wahrscheinlichkeit des Spontanabgangs haben und eine frühzeitige Intervention erfordern. In der Studie von El-Nahas et al. (2009) wurden mehr Misserfolge und Komplikationen bei der Behandlung von proximalen Harnleitersteinen im Vergleich zu distalen Steinen gefunden. Dieses Ergebnis schrieb die Studie einer häufigeren Steinmigration ("push back") und einer höheren Wahrscheinlichkeit für die Entwicklung von Komplikationen im proximalen Ureter zu. Insgesamt waren allerdings $87 \%$ der durchgeführten Behandlungen von Uretersteinen erfolgreich im Sinne einer kompletten Entfernung aller röntgenologisch und optisch sichtbaren Steinfragmente. Preminger (2006) berichtete von der Behandlung solitärer Unterkelchsteine bis $30 \mathrm{~mm}$ mit einem 7,5 Ch (2,5 mm) flexiblen Ureterorenoskop im Rahmen einer multizentrischen Studie. Die Steinfreiheitsrate betrug 3 Monate nach der Behandlung 85\%. Pearle et al. (2008) veröffentlichten eine Studie, in der 35 Patienten mit solitären Steinen $\leq 10 \mathrm{~mm}$ der unteren Nierenkelche mit dem flexiblen Ureterorenoskop behandelt wurden. 3 Monate nach Behandlung waren 50\% der Patienten steinfrei. Smith RD und Patel (2007) beschrieben, dass die ermittelte Steinfreiheitsrate nach Ureterorenoskopie im Hohlsystem in Abhängigkeit von den verwendeten bildgebenden Verfahren va- 
riiere. Mit dem CT (ohne Kontrastmittel) würden postoperativ Restfragmente erkannt, die mit einer Röntgenaufnahme oder Ultraschalluntersuchung möglicherweise übersehen worden wären. Unterschiedliche Erfolgsraten bei der Harnsteinbehandlung im Nierenbeckenkelchsystem könnten sich durch verschiedene Verfahren zur postoperativen Erkennung von Restfragmenten ergeben.

In Göttingen stieg die Zahl der erfolgreichen Ureterorenoskopien an, je weiter distal der behandelte Harnstein lokalisiert war. Abbildung 11 zeigt einen Anstieg der Steinfreiheitsrate ausgehend vom Nierenbeckenkelchsystem mit 49,6\% zum distalen Ureter mit 90\%. Die Entwicklung lässt sich unter anderem damit erklären, dass kleine und damit komplikationsärmere Harnsteine häufig erst vor der Blasenenge im distalen Ureter zu liegen kamen. Größere Steine bleiben eher proximal eingeklemmt (inkarzeriert). Eine höhere Komplikationsrate im proximalen Harntrakt (s. Abbildung 19) führte ebenso zu einer geringeren Rate erfolgreicher Steinbehandlungen.

Steinfreiheit und Steingröße Sözen et al. (2003) fanden, dass die Steinfreiheitsrate bei Steinen $>10 \mathrm{~mm}$ mit $83,7 \%$ geringer war als bei Steinen $\leq 10 \mathrm{~mm}$ mit $97,1 \%$. Ihre Studie bezieht sich auf die Behandlung von 500 Patienten mit dem Lithoclast $\circledast$ Lithotripsiesystem, davon 75,2\% nach erfolgloser ESWL. El-Nahas et al. (2009) publizierten, dass die Rate nicht erfolgreicher und komplikationsreicher Behandlungen mit zunehmender Länge und Breite des Uretersteins zunahm.

In dieser Studie stieg die Steinfreiheitsrate mit abnehmender Steingröße an. Bei Harnsteinen $>10 \mathrm{~mm}$ wurde eine Steinfreiheitsrate von $32 \%$, in der Gruppe $>5 \mathrm{~mm}$ bis $\leq 10$ $\mathrm{mm}$ eine Rate von $64,2 \%$ und in der Gruppe $\leq 5 \mathrm{~mm}$ eine Steinfreiheitsrate von $77 \%$ erreicht.

Steinfreiheit und Patientenalter Ng (2009) verglich anhand der aktuellen wissenschaftlichen Literatur die Ergebnisse der ureterorenoskopischen Steinbehandlung von Kindern und alten Menschen (Personen der „Altersextreme“) mit den Ergebnissen „normaler" erwachsener Patienten. Die Studie konnte keine unterschiedliche Erfolgsrate zwischen den einzelnen Altersgruppen nachweisen.

Tabelle 7 zeigt die erreichten Steinfreiheitsraten für die einzelnen Altersgruppen des Patientenkollektivs in Schritten von 10 Jahren. Da hier kein offensichtlicher Zusammenhang erkennbar war, wurden die Patienten in der Nähe ihres mittleren Alters von 49,7 Jahren in einer Gruppe von Patienten unter 50 Jahren und über 50 Jahren aufge- 
teilt. Die Gruppe der jüngeren Patienten wies in dieser Betrachtung eine um 9,3\% höhere Steinfreiheitsrate als die Vergleichsgruppe auf. Da die Komplikationsrate in dieser Studie bei Patienten $\geq 50$ Jahre gegenüber den Patienten $<50$ Jahre ebenfalls erhöht war (s. Abbildung 20), könnte dies eine niedrigere Steinfreiheitsrate der älteren Patienten zum Teil mitbegründen. Aufgrund der geringen Unterschiede der Altersgruppen in Bezug zur erreichten Steinfreiheit ist ein zufälliges Auftreten dieser Beobachtung nicht auszuschließen.

Steinfreiheit und Body-Mass-Index Dash et al. (2002) konnten bei der ureterorenoskopischen Behandlung von Nierensteinen keinen Unterschied in der erreichten Steinfreiheitsrate bei 16 massiv übergewichtigen Patienten $\left(B M I>40 \mathrm{~kg} / \mathrm{m}^{2}\right)$ im Vergleich zu 38 normalgewichtigen Patienten (BMI 18.5-24,9kg/ $\mathrm{m}^{2}$ ) finden. Knoll et al. (2009 a) empfehlen in den „S2-Leitlinien zur Diagnostik, Therapie und Metaphylaxe der Urolithiasis", dass Harnsteinbildner im Rahmen der Metaphylaxe und Prävention weiterer Steinereignisse einen Body-Mass-Index von 18 bis $25 \mathrm{~kg} / \mathrm{m}^{2}$ und damit Normalgewicht erreichen sollten. Damit wird in dieser Publikation der Body-MassIndex als bedeutend für die Behandlung und Entstehung der Urolithiasis anerkannt. In den Leitlinien zur Harnsteintherapie der "European Association of Urology" aus dem Jahr 2007 (Preminger et al. 2007) wurde zur Verbesserung der aktuellen Studienlage die Miterfassung des Body-Mass-Index der Patienten für zukünftige Untersuchungen empfohlen.

In dieser Studie schwankten die erreichten Steinfreiheitsraten bei normal und übergewichtigen Patienten nur wenig. Bei Patienten mit einem BMI Wert von $>18,5 \mathrm{~kg} / \mathrm{m}^{2}$ zeigte sich ein maximaler Unterschied der Steinfreiheitsraten von $6,1 \%$ bei den Frauen und 3,6\% bei den Männern. Lediglich bei den untergewichtigen Patienten mit einem BMI Wert $<18,5 \mathrm{~kg} / \mathrm{m}^{2}$ wich die Rate mit $60 \%$ deutlich von der durchschnittlichen Steinfreiheitsrate von $70 \%$ ab. Da diese Gruppe mit insgesamt 10 Patienten sehr klein war, ist ein zufällige Beobachtung möglich. In dieser Studie war damit weder eine positive noch negative Beeinflussung der Steinfreiheitsrate durch den Body-Mass-Index des Patienten zu zeigen.

Steinfreiheit und Ureterorenoskope Die erreichten Steinfreiheitsraten variierten deutlich zwischen den eingesetzten Geräten. Das am häufigsten verwendete groBe semirigide Ureterorenoskop wurde als Einzelgerät mit einer Steinfreiheitsrate von 
$81,8 \%$ eingesetzt. Obwohl das kleine semirigide Gerät wesentlich seltener Verwendung fand, wurde auch mit diesem Gerät eine hohe Steinfreiheitsrate von 73,3\% erreicht. Das flexible Ureterorenoskop wurde ebenfalls selten als Einzelinstrument verwendet und erreichte eine Steinfreiheitsrate von 55,8\%. Bei Behandlungen, in denen mehrere Ureterorenoskope eingesetzt wurden, lag die erreichte Steinfreiheitsrate immer unter 60\%. Abbildung 14 zeigt, dass die semirigiden Ureterorenoskope beider Durchmesser am häufigsten im distalen Ureter eingesetzt wurden. Bei Operationen, in denen ausschließlich semirigide Ureterorenoskope Verwendung fanden, wurden in 57\% Harnsteine im distalen Ureter behandelt, die Steinfreiheitsrate betrug 92,9\%. Lediglich $6,1 \%$ der Behandlungen fanden bei Harnsteinen im Nierenbeckenkelchsystem statt, die Erfolgsrate betrug 57,1\%. Flexible Ureterorenoskope wurden insgesamt seltener eingesetzt, meistens in Kombination mit dem großen semirigiden Gerät. Viele der Operationen mit dem flexiblen Ureterorenoskop entfielen auf die Steinbehandlungen im Hohlsystem (57\%), dies mit einer Erfolgsrate von 48,8\%.

In dieser Studie wurden vor allem die semirigiden Ureterorenoskope als Einzelinstrumente sehr erfolgreich eingesetzt. Je mehr Geräte im Verlauf einer Behandlung zum Einsatz kamen, desto geringer war die Erfolgsrate der Operation. Dies galt ebenso für die Verwendung des flexiblen Ureterorenoskops als Einzelinstrument, welches wesentlich weniger erfolgreiche Behandlungen aufwies als die semirigiden Geräte. Während die semirigiden Ureterorenoskope bevorzugt im distalen Harntrakt Verwendung fanden, wurden die flexiblen Geräte häufiger im proximalen Harntrakt eingesetzt. Dies entspricht den von Bagley et al. (2004) genannten bevorzugten Anwendungsbereichen. Die Publikation beschreibt das semirigide Ureterorenoskop als verlässliches Gerät zur Steinbehandlung im distalen Ureter, während das flexible Gerät normalerweise der Behandlung proximaler Harnsteine vorbehalten bleibt. In der vorliegenden Arbeit ist die geringere Steinfreiheitsrate der flexiblen Geräte im Vergleich zu den semirigiden Geräten teilweise mit erschwerten anatomischen Bedingungen im Nierenbeckenkelchsystem und damit einer höheren Komplikationsrate erklärbar (s. Abbildung 19). $\mathrm{Da}$ in dieser Arbeit bei verbliebenen abgangsfähigen Restfragmenten die Operation als „nicht erfolgreich“ galt, war bei einer notwendigen Lithotripsie von einem größeren Risiko für einen Misserfolg auszugehen. Besonders große und proximal gelegene Harnsteine wurden häufig durch die Verwendung von Lithotripsiesystemen vor ihrer Bergung zerkleinert. Die geschah bevorzugt mit dem flexiblen Ureterorenoskop. 
Steinfreiheit und Instrumentarium Bei der Untersuchung der eingesetzten Instrumente zeigte sich, dass das Dormiakörbchen und die Kombination von Ho:YAG-Laser und Dormiakörbchen mit 84,8\% Anteil an den Behandlungen einen dominierenden Anteil einnahm. Andere Instrumente wie Fasszange, Sonotrode und Zeiss'sche Schlinge machten auch in Verbindung mit Laser und Dormiakörbchen einen sehr geringen Teil aus. Es zeigte sich, dass das Dormiakörbchen alleine 353 mal Verwendung fand, während das Körbchen in Verbindung mit dem Laser nur bei 132 Behandlungen eingesetzt wurde. Bei der erreichten Steinfreiheit lag das Körbchen als Einzelinstrument mit $82,7 \%$ deutlich vor der Instrumentenkombination mit 56,1\%. Abbildung 15 verdeutlicht, dass die Instrumente unterschiedlich häufig in den Harntraktabschnitten Verwendung fanden. Das Körbchen alleine wurde im Vergleich zur Kombination aus Körbchen und Laser häufiger im distalen Ureter und seltener im Nierenbeckenkelchsystem eingesetzt. Mit dem Körbchen wurden von 343 Behandlungen $70,6 \%$ bei Steinen $\leq 5 \mathrm{~mm}$ und $29,4 \%$ bei Steinen $>5 \mathrm{~mm}$ und $\leq 10 \mathrm{~mm}$ durchgeführt (s. Abbildung 16). Kein einziger Stein >10 mm wurde mit dem Körbchen behandelt. Die Kombination aus Körbchen und Laser, bei der eine Lithotripsie möglich ist, lieferte eine andere Verteilung. Von 126 Behandlungen fanden $34,1 \%$ bei Steinen $\leq 5 \mathrm{~mm}, 54 \%$ bei Steinen zwischen $>5 \mathrm{~mm}$ und $\leq 10 \mathrm{~mm}$ und $11,9 \%$ bei Steinen $>10 \mathrm{~mm}$ statt.

Dieses Ergebnis lässt sich gut damit erklären, dass die Kombination aus Körbchen und Laser häufig zur Lithotripsie größerer Steine angewendet wurde, während unkomplizierte kleine Steine $\leq 5 \mathrm{~mm}$ sehr häufig in toto mit dem Körbchen geborgen wurden. Die Möglichkeit, die Laserfaser auch in einem flexiblen Ureterorenoskop zu verwenden, wurde genutzt, um proximale Steine zu lithotripsieren. Wie bereits gezeigt wurde (s. Abbildung 11), nimmt bei den Geräten die erreichte Steinfreiheitsrate ab, je weiter proximal eine Steinbehandlung stattgefunden hat. Die geringe Steinfreiheit von $26,7 \%$ bei 15 Behandlungen von Steinen >10 mm sollte nicht darüber hinwegtäuschen, dass hier eine Behandlung ohne Lithotripsiesystem wie dem Ho:YAG-Laser nicht möglich gewesen wäre. Die Verwendung der Zeiss' schen Schlinge, welche in Göttingen zuletzt 1999 Anwendung fand, stellt nach aktueller wissenschaftlicher Meinung keine vertretbare Behandlungsoption mehr dar (Preminger et al. 2007).

Komplikationen In der Literatur werden auftretende Komplikationen nicht einheitlich in die Gruppen Major- und Minorkomplikationen bzw. Früh- und Spätkomplikationen eingeteilt. In den aktuellen „S2-Leitlinien zur Diagnostik, Therapie und Metaphylaxe der Urolithiasis“ aus dem Jahr 2009 (Knoll et al. 2009 b) wurde eine Rate von 3-11\% an 
„signifikanten“ Komplikationen beschrieben. Dazu zählten Sepsis, Ureterperforation und Ureterabriss.

Bei 594 durchgeführten Behandlungen zur Harnsteinsanierung kam es bei 9,9\% zu Komplikationen. Bei 5\% der Behandlungen handelte es sich um eine schwere „Majorkomplikation“. Dies bezieht sich auf die Behandlung von Harnsteinen im gesamten Harntrakt. Bei 402 Steinbehandlungen im Ureter kam es zu einer Gesamtkomplikationsrate von 9,7\% und einer Majorkomplikationsrate von 5,5\%. Diese Ergebnisse bewegen sich im unteren Rahmen der in der Literatur beschriebenen Komplikationsereignisse bei der Ureterorenoskopie.

Komplikationen und Steingröße Fuganti et al. (2008) publizierten eine Studie, in der eine höhere intraoperative Komplikationsrate bei steigender Steingröße nachgewiesen werden konnte.

Die Ergebnisse dieser Studie (s. Abbildung 18) zeigten ebenfalls einen Anstieg der Komplikationsrate bei steigender Steingröße. Die Komplikationsrate bei Harnsteinen $>10 \mathrm{~mm}$ war annähernd doppelt so hoch als bei Steinen $<10 \mathrm{~mm}$, allerdings sollte bei der Beurteilung dieser Beobachtung die geringe Anzahl von 25 Behandlungen bei Steinen >10 mm kritisch gesehen werden. Bei größeren Steinen bestand zusätzlich häufig die Notwendigkeit zur Lithotripsie. Ein größerer apparativer und zeitlicher Aufwand unter schwierigeren Bedingungen im proximalen Harntrakt könnte eine höhere Komplikationsrate und eine höhere Rate an nicht erfolgreichen Ureterorenoskopien erklären. Tatsächlich zeigte sich, dass Harnsteine $>10 \mathrm{~mm}$ in dieser Studie die geringste Steinfreiheitsrate aufwiesen (s. Abbildung 12).

Komplikationen und Steinlokalisation Geavlete et al. (2006) konnten bei 2735 therapeutischen und diagnostischen semirigiden Ureterorenoskopien für die intraoperative Komplikationen einen Unterschied zwischen proximalen und distalen Harnsteinen zeigen. Während 5,6\% der intraoperativen Komplikationen im proximalen Ureter auftraten, waren es im distalen Ureter 3,7\% . In der Studie von Fuganti et al. (2008) stieg die intraoperative Komplikationsrate ebenfalls aufsteigend vom distalen zum proximalen Ureter. Hofmann (2006) beschreibt eine deutlich ansteigende Inzidenz von Perforationskomplikationen im oberen Harntrakt.

In Göttingen zeigte sich eine Gesamtkomplikationsrate von 13,3\% bei Harnsteinen im proximalen, 11,4\% im mittleren und 8,2\% im distalen Ureter (s. Abbildung 19 ). Diese 
Ergebnisse beziehen sich auf 497 (83,7\%) von 594 Behandlungen, bei denen Harnstein(e) in einem isolierten Abschnitt des Harntrakts behandelt wurden. Die intraoperativen Komplikationen Ureterabriss, Ureterperforation und Nierenbeckenperforation wurden im proximalen Harnleiter bei 7 von 75 Behandlungen (9,3\%) und im distalen Ureter bei 5 von 219 Behandlungen (2,3\%) beobachtet. Sowohl bei der Gesamtkomplikationsrate, als auch bei intraoperativen Komplikationen zeigte sich damit ein Anstieg der Komplikationen bei der Harnsteinbehandlung von distal nach proximal.

Komplikationen und Patientenalter In der Studie von Fuganti et al. (2008) konnten für intraoperative Komplikationen der Einfluss des Patientenalters auf die Komplikationsrate dargestellt werden. Es wurde gezeigt, dass mit zunehmendem Alter der Patienten die Rate intraoperativer Komplikationen zunahm. $\mathrm{Ng}$ (2009) allerdings konnte in der wissenschaftlichen Literatur keinen Anhalt dafür finden, dass sehr junge oder sehr alte Patienten eine höhere allgemeine Komplikationsrate als „normale“ erwachsene Patienten aufwiesen.

Die Auftragung der Komplikationsrate bezogen auf die einzelnen Lebensdekaden, in denen sich die Patienten zum Zeitpunkt der Behandlung befanden, zeigte keine eindeutige Tendenz zu mehr Komplikationen bei älteren Patienten (s. Tabelle 11). Dies beruhte möglicherweise auf der geringen Patientenanzahl, die sich für die einzelnen Gruppen ergab. Bei der Aufteilung des Patientenkollektivs in etwa zwei gleich große Gruppen, wobei Patienten $<50$ Jahre und $\geq 50$ Jahre betrachtet wurden, wies die ältere Gruppe eine höhere allgemeine Komplikationsrate auf als die jüngere Gruppe (12,3\% zu 7,5\%, s. Abbildung 20). In der Gruppe der älteren Patienten waren die Harnleiterperforationen $(73,7 \%)$, die Nierenbeckenperforationen $(66,6 \%)$ und das postoperative Fieber $(60 \%)$ häufiger vertreten als bei Patienten unter 50 Jahren. Damit zeigte sich auch in Göttingen tendenziell eine höhere Rate intraoperativer Komplikationen bei älteren Patienten. Keine Altersabhängigkeit zeigten die Komplikationen Harnabflussstörung, transfusionsbedürftige Blutung, Harnleiterabriss und Urosepsis. Die Bedeutung des Patientenalters für die Komplikationsrate schien damit in dieser Studie von der Art der betrachteten Komplikation abzuhängen. Dies entspricht den Ergebnissen der Publikationen von Fuganti et al. (2008), die ebenfalls nicht für alle Komplikation einen Zusammenhang darstellen konnten. 
Komplikationen und Body-Mass-Index Dash et al. (2002) verglichen die Ergebnisse der ureterorenoskopischen Nierensteinbehandlung von 38 normalgewichtigen (BMI $18.5-24,9 \mathrm{~kg} / \mathrm{m}^{2}$ ) mit 16 massiv übergewichtigen (BMI $>40 \mathrm{~kg} / \mathrm{m}^{2}$ ) Patienten. Sie konnten keine erhöhte Komplikationsrate bei den übergewichtigen Steinpatienten nachweisen.

In dieser Studie wurden aufgetretene Komplikationen anhand der WHO Klassifikation des Body-Mass-Index bei Erwachsenen (World Health Organization 2004) ausgewertet. $60 \%$ der Nierenbeckenperforationen ereigneten sich bei „übergewichtigen“ Patienten mit einem BMI Wert von $25-30 \mathrm{~kg} / \mathrm{m}^{2}$, welche einen Anteil von 40,1\% am erwachsenen Patientenkollektiv einnahmen. Eine erhöhte Komplikationsrate von 30\% konnte bei untergewichtigen Patienten $\left(\mathrm{BMl}<18,5 \mathrm{~kg} / \mathrm{m}^{2}\right)$ festgestellt werden. Es zeigte sich ein leichter Abfall der Komplikationsrate bei adipösen erwachsenen Patienten $\left(\mathrm{BMI}>30 \mathrm{~kg} / \mathrm{m}^{2}\right.$ ), die einen Anteil von 27,5\% am Patientenkollektiv ausmachten, aber nur in $15 \%$ ein postoperatives Fieber und in $20 \%$ eine Harnabflussstörung zeigten.

Die vermehrte Beobachtung von Nierenbeckenperforationen bei übergewichtigen $\mathrm{Pa}$ tienten war nicht auf die Harnleiterperforationen zu übertragen, bei denen sich keine solche Häufung fand. Die geringe Zahl von 10 dokumentierten Nierenbeckenperforationen macht eine zufällige Häufung bei dieser Patientengruppe denkbar. Ebenso ist durch die geringe Anzahl von 10 untergewichtigen Patienten der zufällige Anstieg der Komplikationsrate bei dieser Gruppe erklärbar. Die geringere Komplikationsrate bei adipösen Patienten bei postoperativem Fieber und die Entwicklung einer Harnabflussstörung ist auch hier vermutlich auf die nur geringe Anzahl von Komplikationen zurückzuführen. Es zeigte sich allerdings keine einzige Komplikation, die bei adipösen Patienten auffällig häufig vorkam, so dass eine Verbindung zwischen Fettleibigkeit und erhöhter Komplikationsrate bei der URS aus dieser Arbeit nicht hervorgeht.

Komplikationen und Ureterorenoskope Es wurden mit dem semirigiden Ureterorenoskop alleine bzw. in Kombinationen mit dem flexiblen Ureterorenoskop 76,3\% der Behandlungen durchgeführt mit einer Komplikationsrate von 9,5\% im Vergleich zu 19,2\% mit den restlichen Geräten. Die höchste Komplikationsrate von 20\% wurde bei Verwendung des kleinen semirigiden Ureterorenoskops als Einzelinstrument beobachtet.

Es zeigte sich, dass die häufig verwendeten Gerätekonstellationen semirigides Ureterorenoskop alleine und in Kombination mit dem flexiblen Gerät die geringsten Kompli- 
kationsraten aufwiesen. Eine andere Beobachtung wurde bei der Beziehung zwischen eingesetzten Geräten und erreichter Steinfreiheit gemacht. Hier zeigte sich, dass bei intraoperativer Verwendung mehrerer Geräte, zB. semirigides und flexibles Gerät, die Steinfreiheitsraten sanken. Die hohe Komplikationsrate bei Verwendung des kleinen semirigiden Ureterorenoskops erklärt sich zum Teil daraus, dass dieses Gerät einen um 2 Ch $(0,67 \mathrm{~mm})$ dünneren Schaft besitzt als das große Ureterorenoskop und somit besonders bei schwierigen anatomischen Bedingungen wie Harnleiterstenosen Verwendung fand.

Komplikationen und Instrumentarium Das Dormiakörbchen als Einzelinstrument hatte bei 353 Eingriffen eine Komplikationsrate von 8,2\%, verglichen mit 10,6\% bei der Kombination aus Dormiakörbchen und Ho:YAG-Laser bei 132 Einsätzen. Es wurden nur diese beiden Kombinationen ausgewertet, da sie in 84,8\% der auswertbaren Operationen eingesetzt wurden. Bei den Harnabflussstörungen zeigte sich, dass $80 \%$ (8 von 10) bei Operationen mit dem Körbchen als Einzelinstrument auftraten, wobei dieses Instrument nur in 59,4\% der Operationen benutzt wurde.

Die höhere Komplikationsrate bei der Verwendung der Instrumentenkombination aus Dormiakörbchen und Ho:YAG-Laser erklärt sich zum Teil daraus, dass die Verwendung des Lasers zur Lithotripsie vor allem bei großen Steinen unumgänglich ist, was zu einer schlechteren Steinfreiheitsrate und vermehrten Restkonkrementen führte. Eine größere Rate von Patienten mit Reststeinen kann wiederum zur Erklärung der erhöhten Komplikationsrate beitragen. Es zeigte sich weiterhin eine Häufung von Harnabflussstörungen bei der Verwendung des Körbchens als einzelnes Instrument. Eine mögliche Erklärung dafür ist, dass nach unkomplizierter Steinextraktion in toto seltener eine Ureterschiene eingelegt wurde. Nur 2 der 10 Patienten mit Harnabflussstörung hatten postoperativ eine Doppel-J-Ureterschiene erhalten, im Vergleich zu 466 von 586 Patienten (79,5\%) des gesamten Patientenkollektivs (8 Patienten ohne Angabe). Hollenbeck et al. (2001) verglichen Patienten mit und ohne postoperativer Harnleiterschienung in Hinblick auf die nach der Ureterorenoskopie aufgetretenen Ergebnisse und Komplikationen. Die Studie konnte keine erhöhte Inzidenz von Harnabflussstörungen in der Gruppe der Patienten ohne postoperativer Schienung finden. Vor dem Hintergrund des abweichenden Literaturergebnisses, ist ein möglicher Zusammenhang zwischen einer sich entwickelnden Harnabflussstörung und einem Verzicht auf eine postoperative Harnleiterschienung kritisch zu sehen. 


\section{Zusammenfassung}

Die Harnsteinbehandlung mit dem Ureterorenoskop ist ein etabliertes urologisches Verfahren. Kenntnisse über Behandlungsoptionen der Urolithiasis sind vor dem Hintergrund einer steigenden Prävalenz und Inzidenz von Bedeutung.

Die vorliegende Arbeit untersuchte retrospektiv die Ergebnisse und Komplikationen der ureterorenoskopischen Steinbehandlung in der Fachabteilung Urologie des Universitätsklinikums Göttingen. Die Resultate der Eingriffe wurden zur Einschätzung ihrer Vergleichbarkeit und der in Göttingen erbrachten Behandlungsqualität mit bereits publizierten Ergebnissen anderer Einrichtungen verglichen und diskutiert. Als Datenquelle dienten archivierte Patientenakten und radiologische Befunde, die auf Behandlungen zwischen dem 01.01.1998 und 31.12.2008 beruhten.

Es wurden 594 ureterorenoskopische Steinbehandlungen im Ureter und Nierenbeckenkelchsystem bei 509 Patienten durchgeführt. 12,4\% der Patienten wurden mehrfach mit der URS behandelt. Das durchschnittliche Patientenalter betrug 49,8 Jahre. Der mittlere BMI war $27,2 \mathrm{~kg} / \mathrm{m}^{2}$, die mittlere Steingröße betrug $5,4 \mathrm{~mm}$. Es wurden 160 Frauen und 349 Männer behandelt. Die komplette Steinfreiheit ohne Restfragmente in einer Operation gelang bei 70\% der Behandlungen. Bei 402 Behandlungen wurden Harnsteine nur im Ureter behandelt, die Steinfreiheitsrate betrug 81,6\%.

Es wurde bei 497 Behandlungen von Harnsteinen in einem Harntraktabschnitt eine zunehmende Steinfreiheitsrate absteigend vom Hohlsystem zum distalen Ureter festgestellt. Im Nierenbeckenkelchsystem betrug die Erfolgsrate nach einer URS 49,5\%, im proximalen Ureter $65,3 \%$, im mittleren Ureter $76,1 \%$ und im distalen Ureter $90 \%$. Die Steinfreiheitsrate stieg, je kleiner die behandelten Konkremente waren. Steine $>10$ $\mathrm{mm}$ wurden zu $32 \%$ in einer URS erfolgreich behandelt, Steine $>5 \mathrm{~mm}$ und $\leq 10 \mathrm{~mm}$ zu $64,2 \%$ und Steine $\leq 5 \mathrm{~mm}$ zu $77 \%$. Die semirigiden Ureterorenoskope erreichten mit $81,8 \%$ und $73,3 \%$ die höchsten Steinfreiheitsraten. Semirigide Geräte wurden vor allem im distalen, flexible Geräte häufig im proximalen Harntrakt eingesetzt. In 84,8\% der Operationen wurde entweder das Dormiakörbchen alleine, oder in Kombination mit dem Ho:YAG-Laser verwendet. Das Dormiakörbchen wurde häufig bei Steinen $\leq 10 \mathrm{~mm}$ im distalen Harntrakt zur Komplettextraktion verwendet, es erreichte eine Steinfreiheitsrate von 82,7\%. In Kombination mit dem Ho:YAG-Laser wurde das Dormiakörbchen weiter proximal und bei Steinen $>10 \mathrm{~mm}$ nach Lithotripsie eingesetzt, die Steinfreiheitsrate betrug insgesamt $56,1 \%$. 
Komplikationen traten bei 9,9\% aller Behandlungen auf, schwerere Komplikationen in $5 \%$. Es zeigte sich ein erhöhtes Auftreten von Komplikationen bei Steinen $>10 \mathrm{~mm}$ sowie bei proximalen Harnleitersteinen verglichen mit distal gelegenen Steinen. Die häufig verwendeten Ureterorenoskope wiesen die geringste Komplikationsrate auf. Es handelte sich dabei um das semirigide Gerät alleine und die Kombination mit dem flexiblen Gerät. Bei den Instrumenten hatte das Dormiakörbchen alleine mit 8,2\% eine geringere Komplikationsrate als in Verbindung mit dem Ho:YAG-Laser Lithotripsiesystem mit 10,6\%.

Zusammenfassend kann festgestellt werden, dass die Ureterorenoskopie in Göttingen eine komplikationsarme und effektive Methode zur Behandlung von Harnsteinen im Ureter und Hohlsystem darstellte. Die Ergebnisse dieser Arbeit waren in den wesentlichen Punkten Steinfreiheits- und Komplikationsrate vergleichbar mit den Resultaten der internationalen Literatur. Verschiedene mögliche Einflussfaktoren auf die Behandlungsresultate wurden untersucht und diskutiert, dabei zeigten sich teils von Ergebnissen dieser Arbeit abweichende Studienresultate. Weitere Untersuchungen zur Effizienz und optimalen Indikationsstellung der Ureterorenoskopie bei Urolithiasis werden vor dem Hintergrund einer stetigen technischen Weiterentwicklung als sinnvoll erachtet. 


\section{Literaturverzeichnis}

Abdel-Razzak OM, Bagley DH (1992): Clinical experience with flexible ureteropyeloscopy. J Urol 148, 1788-1792

Adeghate E, Schattner P, Dunn E (2006): An update on the etiology and epidemiology of diabetes mellitus. Ann N Y Acad Sci 1084, 1-29

Bagley DH, Kuo RL, Zeltser IS (2004): An update on ureteroscopic instrumentation for the treatment of urolithiasis. Curr Opin Urol 14, 99-106

Calvert RC, Burgess NA (2005): Urolithiasis and obesity: metabolic and technical considerations. Curr Opin Urol $\underline{15}, 113-117$

Conlin MJ, Marberger M, Bagley DH (1997): Ureteroscopy. Development and instrumentation. Urol Clin North Am 24, 25-42

Dash A, Schuster TG, Hollenbeck BK, Faerber GJ, Wolf JS (2002): Ureteroscopic treatment of renal calculi in morbidly obese patients: a stone-matched comparison. Urology $\underline{60}$, 393-397

Dretler SP, Watson G, Parrish JA, Murray S (1987): Pulsed dye laser fragmentation of ureteral calculi: initial clinical experience. J Urol 137, 386-389

El-Nahas AR, El-Tabey NA, Eraky I, Shoma AM, El-Hefnawy AS, El-Assmy AM, Soliman S, Youssef RF, El-Kenawy MR, Shokeir AA (2009): Semirigid ureteroscopy for ureteral stones: a multivariate analysis of unfavorable results. J Urol 181, 1158-1162

Fuchs GJ (2006): Milestones in endoscope design for minimally invasive urologic surgery: the sentinel role of a pioneer. Surg Endosc 20, $493-499$

Fuganti PE, Pires S, Branco R, Porto J (2008): Predictive factors for intraoperative complications in semirigid ureteroscopy: analysis of 1235 ballistic ureterolithotripsies. Urology $\underline{72}, 770-774$

Geavlete P, Georgescu D, Nita G, Mirciulescu V, Cauni V (2006): Complications of 2735 retrograde semirigid ureteroscopy procedures: a single-center experience. J En- 
dourol 20, $179-185$

Gellner R, Domschke W (2008): Epidemiology of obesity. Chirurg 79, 807-818

Gerber GS, Stockton BR (2006): Use of stents after ureteroscopic stone removal. J Endourol 20, 383-385

Goodman TM (1977): Ureteroscopy with pediatric cystoscope in adults. Urology $\underline{9}, 394$

Grasso M, Bagley D (1994): A 7.5/8.2 F actively deflectable, flexible ureteroscope: a new device for both diagnostic and therapeutic upper urinary tract endoscopy. Urology $\underline{43}, 435-441$

Hesse A, Brändle E, Wilbert D, Köhrmann KU, Alken P (2003): Study on the prevalence and incidence of urolithiasis in Germany comparing the years 1979 vs. 2000. Eur Urol $\underline{44}, 709-713$

Hofmann R (2006): Ureteroscopy (URS) for ureteric calculi. Urologe A $\underline{45}, 637-647$

Hollenbeck BK, Schuster TG, Faerber GJ, Wolf JS (2001): Routine placement of ureteral stents is unnecessary after ureteroscopy for urinary calculi. Urology $\underline{57}, 639-643$

Hollingsworth JM, Rogers MA, Kaufman SR, Bradford TJ, Saint S, Wei JT, Hollenbeck BK (2006): Medical therapy to facilitate urinary stone passage: a meta-analysis. Lancet $\underline{368}, 1171-1179$

Knoll T, Alken P (2006): Ureterorenoscopy: yesterday, today, tomorrow. Urologe A $\underline{45}$, 185-186

Knoll T, Köhrmann KU, Fahlenkamp D, Janitzky V, Lahme S, Lebentrau S, Oelschläger S, Türk C, Rassweiler J, Chaussy C (2009 a): S2 Leitlinien zur Diagnostik, Therapie und Metaphylaxe der Urolithiasis. Link: http://www.uni-duesseldorf.de/AWMF/II/ 043-025.pdf (letzter Zugriff 18.03.2010)

Knoll T, Köhrmann KU, Fahlenkamp D, Janitzky V, Lahme S, Lebentrau S, Oelschläger S, Türk C, Rassweiler J, Chaussy C (2009 b): S2 guidelines on diagnostic, therapy and 
metaphylaxis of urolithiasis : Part 1: Diagnostic and therapy. Urologe A $\underline{48}, 917-924$

Lamskemper D: Analyse der ureterorenoskopischen Steintherapie von 1998 bis 2004 unter Berücksichtigung verwendeter Ureteroskope, der Harnleiterkatheterisierung sowie der Nachsorge und Metaphylaxe: Eine retrospektive Arbeit mit Patientenbefragung. Med. Diss. Münster 2006

$\mathrm{Ng}$ CF (2009): The effect of age on outcomes in patients undergoing treatment for renal stones. Curr Opin Urol $\underline{19}, 211-214$

Pearle MS, Lingeman JE, Leveillee R, Kuo R, Preminger GM, Nadler RB, Macaluso J, Monga M, Kumar U, Dushinski J (2005): Prospective randomized trial comparing shock wave lithotripsy and ureteroscopy for lower pole caliceal calculi $1 \mathrm{~cm}$ or less. $J$ Urol 173, 2005-2009

Perez-Castro Ellendt E, Martinez-Pineiro JA, Beneitez M, Mora Durban MM (1984): Transurethral ureter-renoscopy. Past, present and future. Arch Esp Urol 37, 725-740

Preminger GM (2006): Management of lower pole renal calculi: shock wave lithotripsy versus percutaneous nephrolithotomy versus flexible ureteroscopy. Urol Res $\underline{34}$, 108-111

Preminger GM, Tiselius HG, Assimos DG, Alken P, Buck AC, Gallucci M, Knoll T, Lingeman JE, Nakada SY, Pearle MS (2007): 2007 Guideline for the management of ureteral calculi. Eur Urol $\underline{52}, 1610-1631$

Prugger C, Heuschmann PU, Keil U (2006): Epidemiology of hypertension in Germany and worldwide. Herz 31, 287-293

Sakhaee K (2008): Nephrolithiasis as a systemic disorder. Curr Opin Nephrol Hypertens $\underline{17}, 304-309$

Schubert G (2006): Stone analysis. Urol Res $\underline{34}, 146-150$

Smith DG (2007): Epidemiology of dyslipidemia and economic burden on the healthcare system. Am J Manag Care $\underline{13}, 68-71$

Smith RD, Patel A (2007): Impact of flexible ureterorenoscopy in current management 
of nephrolithiasis. Curr Opin Urol 17, 114-119

Sözen S, Küpeli B, Tunc L, Senocak C, Alkibay T, Karaoglan U, Bozkirli I (2003): Management of ureteral stones with pneumatic lithotripsy: report of 500 patients. J Endourol $\underline{17}, 721-724$

World Health Organization (2004): BMI classification. Link: http://apps.who.int/bmi/ index.jsp?introPage=intro_3.html (letzter Zugriff 10.03.2010) 


\section{Danksagung}

Bei Herrn Prof. Dr. med. Rolf-Hermann Ringert, dem Direktor der Abteilung Urologie der Georg-August-Universität Göttingen, möchte ich mich für die Möglichkeit bedanken, in seiner Abteilung eine Promotionsarbeit anfertigen zu dürfen.

Herrn PD Dr. med. Hagen Loertzer, dem leitenden Oberarzt der Urologischen Universitätsklinik, danke ich sehr für die Überlassung des Themas und die Betreuung dieser Arbeit. Ich möchte mich darüber hinaus für seine Unterstützung bei deren Verwirklichung bedanken.

Bei Herrn Dr. med. Arne Strauß, Oberarzt der Urologischen Universitätsklinik, bedanke ich mich für seine Hilfe bei komplizierten Sachfragen und seine Bereitschaft, konstruktiv Kritik zu üben.

Mein besonderer Dank gilt Herrn Klaus-Dieter Plothe, dem leitenden Pfleger der Urologischen Poliklinik. Mit großer Hilfsbereitschaft, Geduld und langjähriger Berufserfahrung war er maßgeblich daran beteiligt, dass die Arbeit gut voranschritt und mit einem stetigen Erkenntnisgewinn für mich verbunden war. Zahlreiche Abbildungen aus seinem Privatarchiv, welche er freundlicherweise zur Verfügung stellte, bereichern diese Arbeit um wichtige Eindrücke aus der urologischen Praxis.

Ich möchte mich weiter bei dem gesamten Team der urologischen Poliklinik bedanken, das mir gegenüber stets freundlich und hilfsbereit war. 


\section{Lebenslauf}

Am 23. August 1985 wurde ich, Philipp-Paul Lumma, als Sohn des Arztes Paul Lumma und der technischen Bauzeichnerin Beate Lumma in Paderborn geboren.

Von April 1992 bis April 1996 besuchte ich die Grundschule in Bad Driburg, anschlieBend wechselte ich auf das Städtische Gymnasium. Im Juni 2004 erlangte ich das Abitur mit der Allgemeinen Hochschulreife am Städtischen Gymnasium Bad Driburg.

Von Oktober 2004 bis August 2005 leistete ich meinen Wehrdienst bei der Deutschen Marine ab. Von Januar bis August 2005 war ich als Gefreiter im Fernmeldebetrieb an Bord des Versorgungsschiffs „Frankfurt am Main“ tätig.

Im Wintersemester 2005/2006 begann ich mein Studium der Humanmedizin an der Georg-August-Universität Göttingen. Nach vier vorklinischen Semestern bestand ich im Sommersemester 2007 das Physikum. Seit September 2008 arbeite ich parallel zur klinischen Ausbildung an der Georg-August-Universität Göttingen an meiner Promotion. 